\title{
Esquema compacto de diferenças finitas de alta ordem em malhas hierárquicas
}

\author{
Ellen Thais Alves Cerciliar
}

\section{DISSERTAÇÃO APRESENTADA}

$\mathrm{AO}$

Instituto DE MATEMÁticA E ESTATÍSTICA

DA

Universidade DE SÃo PAUlo

PARA

OBTENÇÃO DO TÍTULO

$\mathrm{DE}$

MESTRE EM CiÊNCIAS

\author{
Programa: Matemática Aplicada \\ Orientador: Prof. Dr. Alexandre Roma
}

Durante o desenvolvimento deste trabalho o autor recebeu auxílio financeiro do CNPq 


\section{Esquema compacto de diferenças finitas de alta ordem em malhas hierárquicas}

Esta versão da dissertação contém as correções e alterações sugeridas pela Comissão Julgadora durante a defesa da versão original do trabalho, realizada em 21/12/2017. Uma cópia da versão original está disponível no Instituto de Matemática e Estatística da Universidade de São Paulo.

Comissão Julgadora:

- Prof. Dr. Alexandre Megiorin Roma (orientador) - IME-USP

- Prof. Dr. Aristeu da Silveira Neto - FEMEC - UFU

- Prof. Dr. Pedro da Silva Peixoto - IME - USP 


\section{Agradecimentos}

À Deus, o único digno de toda honra e toda glória!

Ao Prof. Dr. Alexandre Megiorin Roma, meu orientador, agradeço pelos ensinamentos, paciência e por exigir sempre que o melhor fosse feito.

À minha família, meus pais Belkiss e Salmista, pelo amor incondicional, pela educação, apoio e exemplo, ao meu esposo José Elias pelo carinho, companheirismo e incentivo, à minha irmã Mara pelo apoio e por sempre me ouvir nos momentos difíceis.

Ao Prof. Dr. Aristeu da Silveira Neto (FEMEC-UFU) por disponibilizar os recursos do MFLab durante a escrita dessa dissertação.

Ao Prof. Dr. Santos Alberto Enriquez Remigio (FAMAT-UFU), pela amizade, pelas ricas sugestões e disposição em ajudar, transmitindo os seus conhecimentos e incentivando minhas conquistas.

Aos membros da banca, agradeço por aceitarem o convite e por todas as considerações, críticas, sugestões e pareceres.

Agradeço igualmente à todos meus queridos amigos e companheiros de estudo, em especial aos que se fizeram mais presentes nessa caminhada, Carla, Rosângela e Nils pelo apoio e torcida pelo meu sucesso.

A todos os professores, funcionários e alunos do IME-USP pela boa convivência e pelo apoio durante a realização desse mestrado.

Ao CNPq, pelo suporte financeiro para a realização desse projeto de pesquisa.

Agradeço à todos que passaram pela minha vida e contribuíram para a construção de quem sou hoje. 
"Divide as dificuldades que tenhas de examinar em tantas partes quantas for possível, para uma melhor solução." 


\section{Resumo}

CERCILIAR, E. T. A. Esquema compacto de diferenças finitas de alta ordem em malhas hierárquicas. 2017. Dissertação (Mestrado) - Instituto de Matemática e Estatística, Universidade de São Paulo, São Paulo, 2017.

Este trabalho propõe um esquema de diferenças finitas compacta de alta ordem para resolver problemas elípticos com coeficientes variáveis em malhas composta. São apresentados a formulação matemática e a dedução do método compacto de quarta ordem aplicado à problemas elípticos bidimensionais, em malha regular e composta. Foi adotado o uso da biblioteca PETSc com os seus pré-condicionadores e métodos numéricos para resolver os sistemas lineares resultantes da discretização do problema. Por fim, testes visando verificar o código foram feitos, utilizando o método de soluções manufaturadas, para mostrar alta eficiência e acurácia do método desenvolvido.

Palavras-chave: Equações elípticas, método das diferenças finitas compacta, malhas hierárquicas. 


\section{Abstract}

CERCILIAR, E. T. A. Higher-order finite-difference schemes for hierarchical meshes. 2017. Dissertação (Mestrado) - Instituto de Matemática e Estatística, Universidade de São Paulo, São Paulo, 2017.

This paper proposes a scheme of compact finite difference higher order for solve elliptic problems with variable coefficients in composite meshes. we present the mathematical formulation and the deduction of the compact method of fourth order applied to two-dimensional elliptic problems in regular and composite mesh. It was adopted using the PETSc library with its pre- conditioners and numerical methods for solving linear systems resulting from discretization of the problem. Finally, tests to verify the code were made using the method of manufactured solutions to show high efficiency and accuracy of the method developed .

Keywords: Elliptic equations, Compact finite difference methods, Hierarchical meshes. 


\section{Sumário}

$\begin{array}{ll}\text { Lista de Figuras } & \text { ix }\end{array}$

Lista de Tabelas $\quad$ xi

1 Introdução $\quad 1$

1.1 Motivação . . . . . . . . . . . . . . . . . . . 1

1.2 Métodos Numéricos de Alta Ordem . . . . . . . . . . . . . . . 2

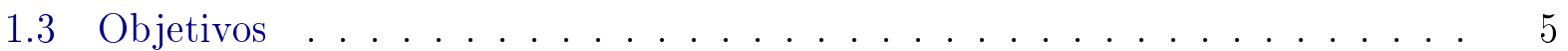

2 Estrutura HiGTree $\quad 7$

2.1 Malhas hierárquicas . . . . . . . . . . . . . . . 7

2.2 Diferenças finitas generalizadas . . . . . . . . . . . . . . 7

2.3 Interpolações . . . . . . . . . . . . . . . . . . . . . . 10

2.3.1 Aproximação polinomial . . . . . . . . . . . . . . . . 10

2.3.2 Aproximação pelo Método dos Mínimos Quadrados . . . . . . . . . . 11

2.3.3 Cálculo dos coeficientes . . . . . . . . . . . . . . . . 12

2.3.4 Diferenças Finitas Generalizadas . . . . . . . . . . . . . 13

3 Metodologia numérica $\quad 15$

3.1 Modelagem Matemática . . . . . . . . . . . . . . . . 15

3.2 Equações Diferencias Parciais . . . . . . . . . . . . . . . . 15

3.3 Equação de Poisson no Método de Projeção . . . . . . . . . . . . . . . 17

3.3.1 Um método explícito de primeira ordem no tempo . . . . . . . . . . 17

3.4 Formulação de métodos . . . . . . . . . . . . . . . . . . 18

3.5 Esquema compacto de diferenças finitas . . . . . . . . . . . . . 18

3.6 Equação elíptica 2D . . . . . . . . . . . . . . . . . . . 19

3.6.1 Formulação do esquema compacto de alta ordem . . . . . . . . . . . 19

4 Resultados e discussões $\quad 25$

4.1 Normas e análise de convergência numérica em malhas regulares e em malhas

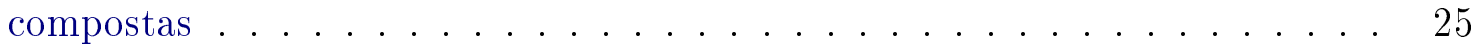

4.2 Representação matricial das discretizações . . . . . . . . . . . . . . . 26

4.3 Problema elíptico com coeficientes constantes . . . . . . . . . . . . 27 
4.3.1 Teste para malha uniforme . . . . . . . . . . . . . . 27

4.3.2 Teste para malha composta . . . . . . . . . . . . 28

4.4 Problema elíptico com coeficientes variáveis . . . . . . . . . . . . 31

4.4.1 Teste para malha uniforme . . . . . . . . . . . . . . . 32

4.4 .2 Teste para malha composta . . . . . . . . . . . . . 34

5 Conclusões $\quad 39$

5.1 Considerações Finais . . . . . . . . . . . . . . . . . . 39

5.2 Sugestões para Pesquisas Futuras . . . . . . . . . . . . . . . 39

A Verificação da ordem de convergência $\quad 41$

A.1 Testes referente ao problema $(4.8) \ldots \ldots \ldots$. . . . . . . . . . 41

A.1.1 Tolerância igual a $1 e-16$ e ordem do polinômio intepolador variando

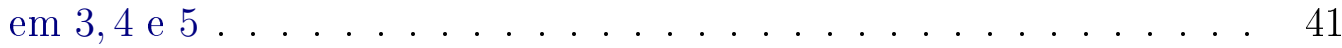

A.1.2 Tolerância igual a $1 e-13$ e ordem do polinômio intepolador variando

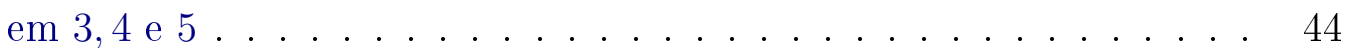

A.1.3 Tolerância igual a $1 e-10$ e ordem do polinômio intepolador variando

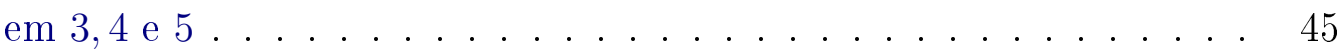

A.1.4 Tolerância igual a $1 e-7$ e ordem do polinômio intepolador variando em 3,4 e $5 \ldots \ldots \ldots \ldots$. . . . . . . . . . . . . . 47

A.2 Testes referente ao problemas $(4.16) \ldots \ldots \ldots \ldots \ldots$. . . . . . . 48

A.2.1 Tolerância igual a $1 e-16$ e ordem do polinômio intepolador variando

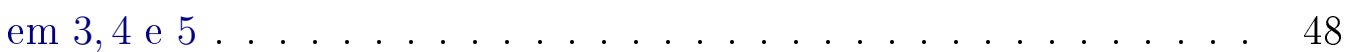

A.2.2 Tolerância igual a $1 e-13$ e ordem do polinômio intepolador variando em 3,4 e $5 \ldots \ldots \ldots \ldots \ldots$. . . . . . . . . . . . . 50

A.2.3 Tolerância igual a $1 e-10$ e ordem do polinômio intepolador variando

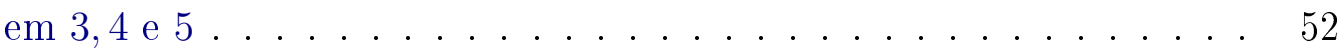

A.2.4 Tolerância igual a $1 e-7$ e ordem do polinômio intepolador variando

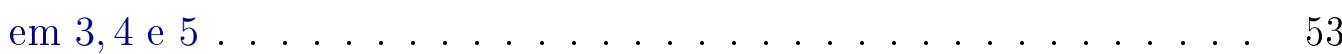

A.2.5 Tolerância igual a $1 e-16$ e ordem do polinômio intepolador variando em 3,4 e $5 \ldots \ldots \ldots \ldots \ldots \ldots \ldots$

A.2.6 Tolerância igual a $1 e-13$ e ordem do polinômio intepolador variando em 3,4 e $5 \ldots \ldots \ldots \ldots \ldots \ldots$

A.2.7 Tolerância igual a $1 e-10$ e ordem do polinômio intepolador variando em 3,4 e $5 \ldots \ldots \ldots \ldots \ldots \ldots$

A.2.8 Tolerância igual a $1 e-7$ e ordem do polinômio intepolador variando em 3,4 e $5 \ldots \ldots \ldots \ldots \ldots \ldots$ 


\section{Lista de Figuras}

2.1 Malha deslocada. . . . . . . . . . . . . . . . . 8

2.2 Exemplo de uma malha tipo HiG e sua representação na forma de árvore

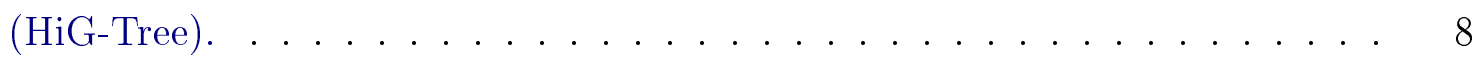

2.3 Exemplo de um estêncil resultando em pontos a serem interpolados $\left(x_{L}\right.$ e $\left.x_{B}\right) . \quad 9$

4.1 Esquema de interpolação . . . . . . . . . . . . . . . . . . . . . . . . . . 29

4.2 Malha correspondente ao testes das tabelas $4.2,4.3,4.4 \ldots \ldots \ldots \ldots$

4.3 Malha uniforme e solução obtida pelo PETSc . . . . . . . . . . . . 32

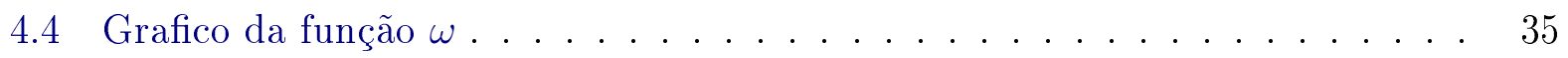

4.5 Malha composta com dois níveis de refinamento e solução obtida pelo PETSc 35

A.1 (a) Malha G1L3, (b) Malha G2L3, (c) Malha G3L3 e (d) Malha G4L3 e (e) Malha G5L3 . . . . . . . . . . . . . . . . . . 42

A.2 (a) Malha G1L1, (b) Malha G2L1, (c) Malha G3L1 e (d) Malha G4L1 e (e) Malha G5L1 . . . . . . . . . . . . . . . . . . . 49

A.3 (a) Malha G1L2, (b) Malha G2L2, (c) Malha G3L2 e (d) Malha G4L2 e (e) Malha G5L2 . . . . . . . . . . . . . . . . . 56 


\section{Lista de Tabelas}

3.1 Definições de operadores de derivadas mistas em uma malha uniforme . . . . 20

4.1 Erro, ordem de convergência e número de iterações do MDF comparado com o ECDF para o problema de Poisson em uma malha uniforme. . . . . . . . .

4.2 Erro dos métodos MDF de quarta ordem e ECDF de quarta ordem em malha composta com ordem do polinômio interpolador igual a $5 . \quad$. . . . . . . 30

4.3 Erro dos métodos MDF de quarta ordem e ECDF de quarta ordem em malha composta com ordem do polinômio interpolador igual a $4 . \quad$. . . . . . . .

4.4 Erro dos métodos MDF de quarta ordem e ECDF de quarta ordem em malha composta com ordem do polinômio interpolador igual a $3 . \quad$. . . . . . . 31

4.5 Erro dos métodos MDF de quarta ordem e ECDF de quarta ordem em malha uniforme com ordem do polinômio interpolador igual a $5 . \quad$. . . . . . .

4.6 Erro dos métodos MDF de quarta ordem e ECDF de quarta ordem em malha uniforme com ordem do polinômio interpolador igual a $4 . \quad$. . . . . . . . 33

4.7 Erro dos métodos MDF de quarta ordem e ECDF de quarta ordem em malha uniforme com ordem do polinômio interpolador igual a $3 . \quad$. . . . . . .

4.8 Erro dos métodos MDF de quarta ordem e ECDF de quarta ordem em malha composta com ordem do polinômio interpolador igual a $5 . \ldots . . . .$.

4.9 Erro dos métodos MDF de quarta ordem e ECDF de quarta ordem em malha composta com ordem do polinômio interpolador igual a 4. . . . . . . . .

4.10 Erro dos métodos MDF de quarta ordem e ECDF de quarta ordem em malha composta com ordem do polinômio interpolador igual a $3 . \quad$. . . . . . .

A.1 Erro e ordem de convergência dos métodos MDF de quarta ordem e ECDF de quarta ordem em malha composta com ordem do polinômio interpolador

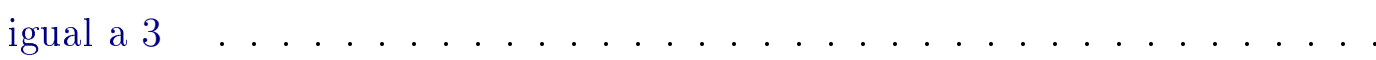

A.2 Erro e ordem de convergência dos métodos MDF de quarta ordem e ECDF de quarta ordem em malha composta com ordem do polinômio interpolador

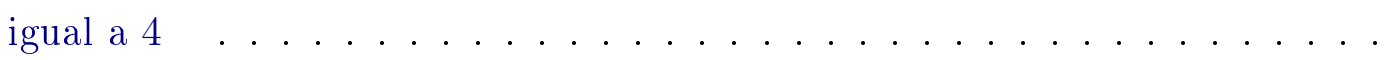

A.3 Erro e ordem de convergência dos métodos MDF de quarta ordem e ECDF de quarta ordem em malha composta com ordem do polinômio interpolador

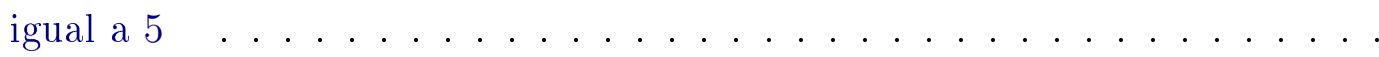


A.4 Erro e ordem de convergência dos métodos MDF de quarta ordem e ECDF de quarta ordem em malha composta com ordem do polinômio interpolador

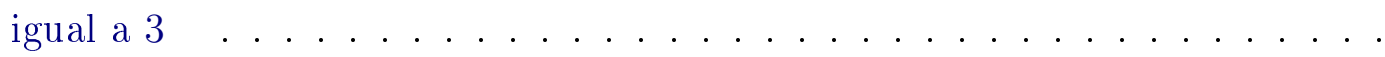

A.5 Erro e ordem de convergência dos métodos MDF de quarta ordem e ECDF de quarta ordem em malha composta com ordem do polinômio interpolador igual a 4

A.6 Erro e ordem de convergência dos métodos MDF de quarta ordem e ECDF de quarta ordem em malha composta com ordem do polinômio interpolador igual a 5

A.7 Erro e ordem de convergência dos métodos MDF de quarta ordem e ECDF de quarta ordem em malha composta com ordem do polinômio interpolador igual a 3

A.8 Erro e ordem de convergência dos métodos MDF de quarta ordem e ECDF de quarta ordem em malha composta com ordem do polinômio interpolador igual a 4

A.9 Erro e ordem de convergência dos métodos MDF de quarta ordem e ECDF de quarta ordem em malha composta com ordem do polinômio interpolador

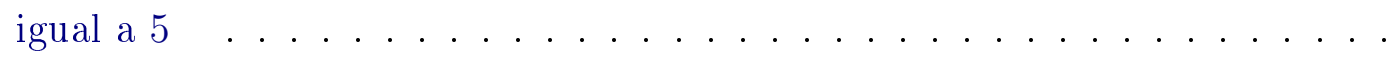

A.10 Erro e ordem de convergência dos métodos MDF de quarta ordem e ECDF de quarta ordem em malha composta com ordem do polinômio interpolador igual a 3

A.11 Erro e ordem de convergência dos métodos MDF de quarta ordem e ECDF de quarta ordem em malha composta com ordem do polinômio interpolador igual a 4

A.12 Erro e ordem de convergência dos métodos MDF de quarta ordem e ECDF de quarta ordem em malha composta com ordem do polinômio interpolador igual a 5

A.13 Erro e ordem de convergência dos métodos MDF de quarta ordem e ECDF de quarta ordem em malha uniforme com ordem do polinômio interpolador igual a 3

A.14 Erro e ordem de convergência dos métodos MDF de quarta ordem e ECDF de quarta ordem em malha uniforme com ordem do polinômio interpolador igual a 4

A.15 Erro e ordem de convergência dos métodos MDF de quarta ordem e ECDF de quarta ordem em malha uniforme com ordem do polinômio interpolador igual a $5 \ldots \ldots \ldots \ldots \ldots$

A.16 Erro e ordem de convergência dos métodos MDF de quarta ordem e ECDF de quarta ordem em malha uniforme com ordem do polinômio interpolador igual a 3 
A.17 Erro e ordem de convergência dos métodos MDF de quarta ordem e ECDF de quarta ordem em malha uniforme com ordem do polinômio interpolador

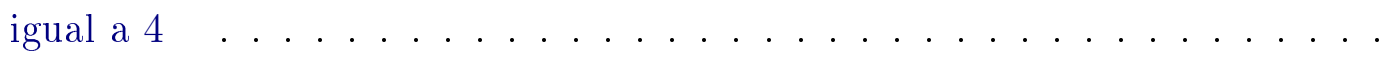

A.18 Erro e ordem de convergência dos métodos MDF de quarta ordem e ECDF de quarta ordem em malha uniforme com ordem do polinômio interpolador

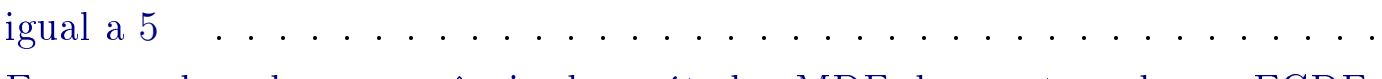

A.19 Erro e ordem de convergência dos métodos MDF de quarta ordem e ECDF de quarta ordem em malha uniforme com ordem do polinômio interpolador igual a 3

A.20 Erro e ordem de convergência dos métodos MDF de quarta ordem e ECDF de quarta ordem em malha uniforme com ordem do polinômio interpolador igual a 4

A.21 Erro e ordem de convergência dos métodos MDF de quarta ordem e ECDF de quarta ordem em malha uniforme com ordem do polinômio interpolador igual a 5

A.22 Erro e ordem de convergência dos métodos MDF de quarta ordem e ECDF de quarta ordem em malha uniforme com ordem do polinômio interpolador

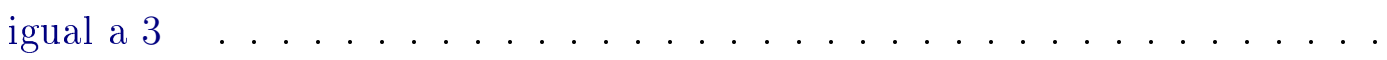

A.23 Erro e ordem de convergência dos métodos MDF de quarta ordem e ECDF de quarta ordem em malha uniforme com ordem do polinômio interpolador

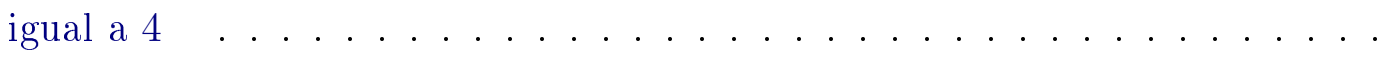

A.24 Erro e ordem de convergência dos métodos MDF de quarta ordem e ECDF de quarta ordem em malha uniforme com ordem do polinômio interpolador igual a 5

A.25 Erro e ordem de convergência dos métodos MDF de quarta ordem e ECDF de quarta ordem em malha composta com ordem do polinômio interpolador igual a 3

A.26 Erro e ordem de convergência dos métodos MDF de quarta ordem e ECDF de quarta ordem em malha composta com ordem do polinômio interpolador igual a 4

A.27 Erro e ordem de convergência dos métodos MDF de quarta ordem e ECDF de quarta ordem em malha composta com ordem do polinômio interpolador igual a 5

A.28 Erro e ordem de convergência dos métodos MDF de quarta ordem e ECDF de quarta ordem em malha composta com ordem do polinômio interpolador

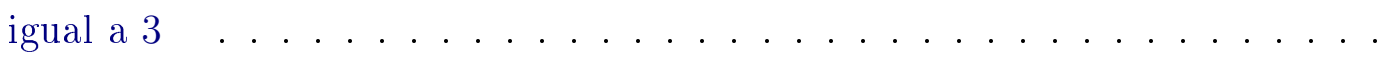

A.29 Erro e ordem de convergência dos métodos MDF de quarta ordem e ECDF de quarta ordem em malha composta com ordem do polinômio interpolador igual a 4 
A.30 Erro e ordem de convergência dos métodos MDF de quarta ordem e ECDF de quarta ordem em malha composta com ordem do polinômio interpolador

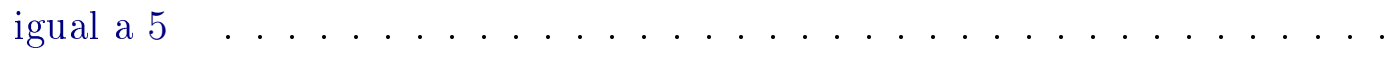

A.31 Erro e ordem de convergência dos métodos MDF de quarta ordem e ECDF de quarta ordem em malha composta com ordem do polinômio interpolador

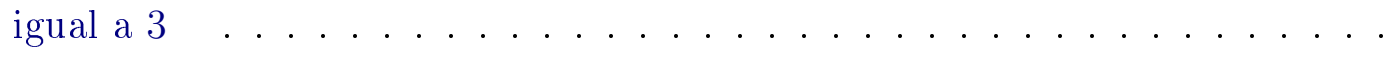

A.32 Erro e ordem de convergência dos métodos MDF de quarta ordem e ECDF de quarta ordem em malha composta com ordem do polinômio interpolador igual a $4 \ldots \ldots \ldots \ldots \ldots \ldots \ldots \ldots \ldots \ldots \ldots \ldots \ldots$

A.33 Erro e ordem de convergência dos métodos MDF de quarta ordem e ECDF de quarta ordem em malha composta com ordem do polinômio interpolador

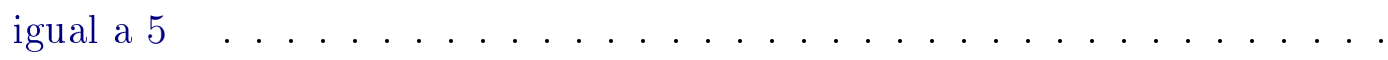

A.34 Erro e ordem de convergência dos métodos MDF de quarta ordem e ECDF de quarta ordem em malha composta com ordem do polinômio interpolador

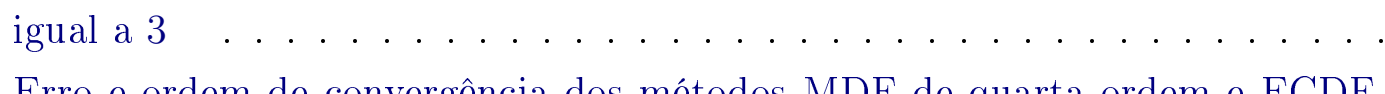

A.35 Erro e ordem de convergência dos métodos MDF de quarta ordem e ECDF de quarta ordem em malha composta com ordem do polinômio interpolador

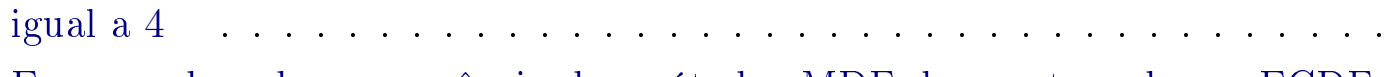

A.36 Erro e ordem de convergência dos métodos MDF de quarta ordem e ECDF de quarta ordem em malha composta com ordem do polinômio interpolador

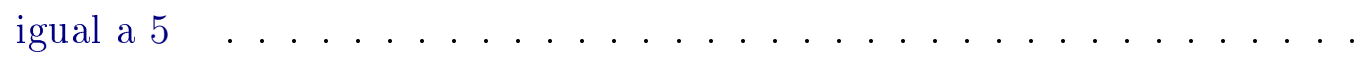




\section{Capítulo 1}

\section{Introdução}

\subsection{Motivação}

A pesquisa em torno de simulações computacionais tem ganhado espaço na literatura ao longo dos anos, pois auxiliam no entendimento de problemas em diversos ramos da engenharia e da ciência. Alguns exemplos podem ser citados nas áreas meteorológica, aerodinâmica, biologia, combustão e muitas outras. Em alguns problemas físicos é demandado um enorme esforço e custo para fazer experimentos em laboratório e muitos recursos são investidos para o desenvolvimento de novas tecnologias, computacional e experimental, com isso, a modelagem matemática e a simulação numérica vêm ganhando muito espaço. Este é o método chamado Dinâmica dos Fluidos Computacional ou CFD (Computational Fluid Dynamics) em inglês.

De forma geral, o usuário da CFD está interessado em obter as distribuições de velocidades, pressões, densidades tensões, temperaturas etc., na região do escoamento. Segundo [5] muito tem se investido em modelos matemáticos para predizer escoamentos, temperaturas, concentrações de espécies químicas de forma a evitar efeitos nocivos ao meio ambiente.

Com simulações em computador pode-se acelerar o desenvolvimento de um projeto, por meio da redução do tempo de testes em túneis de vento, por exemplo. Como todos os parâmetros simulados são virtuais, a princípio basta alterá-los e executar novamente o programa de simulação [9]. Para visualização de campos vetoriais tridimensionais, uma variedade de técnicas foi desenvolvida no passado. Estas técnicas incluem métodos baseados em geometria, como linhas de corrente e partículas animadas, que lembram o que os cientistas estão habituados a ver nos seus experimentos [29].

Em problemas nos quais, por exemplo, há fortes anisotropias de espaçamento nas malhas espaciais ou quando há saltos "grandes"de propriedades materiais, que é o caso da massa específica em escoamentos bifásicos contendo gotas de água em ar (bolhas de ar em água), a implementação é mais complicada [2]. Neste caso, a simulação em malha composta é de grande de interesse e tem recebido contínua atenção dos grupos em CFD do IME-USP e do MfLab/FEMEC-UFU em muitas de suas linhas de pesquisa. Ainda hoje, se faz presente a necessidade de alternativas robustas e eficazes nestas situações onde potencialmente encontramos problemas na convergência dos métodos numéricos.

Condiderando as dificuldades mencionadas acima, tornou-se evidente a necessidade de investirgarmos e propormos uma nova metodologia.

A intenção deste trabalho é desenvolver um método de alta precisão aplicável na solução de equações diferenciais parciais elípticas resultante do processo de correção da pressão nas equações de Navier-Stokes, como foi discutido no trabalho [2] e [44], assim como investigar e acoplar técnicas de discretização espacial e solução dos sistemas lineares gerados por estas 
discretizações.

Para isto, objetiva-se aqui o desenvolvimento de uma metodologia numérica voltada para a solução da equação elíptica em um domínio bidimensional com refinamento adaptativo, que reduzam o tempo computacional, aumentem a robustez do código e aumente a precisão da solução.

Como este estudo é inicial, propoe-se aqui a implementação desta técnica em um domínio bidimensional com refinamento localizado. Uma variedade de testes de verificação por soluções manufaturadas é apresentada neste trabalho exemplificando o potencial desta metodologia numérica.

\subsection{Métodos Numéricos de Alta Ordem}

Métodos numéricos eficientes para gerar aproximações numéricas de Problemas de Valor Inicial (PVI) e / ou de Fronteira (PVF) modelados por equações diferenciais tem sido um objetivo de muitos pesquisadores há décadas. Nos últimos cinquenta anos, três abordagens dominaram as aproximações numéricas: diferenças finitas, elementos finitos e métodos de equações integrais. Entre 1950 e 1980, o Método de Diferenças Finitas (MDF) liderou sua popularidade, foi quando sua teoria e aplicação foi intensamente desenvolvida. Nos últimos anos, o método de elementos finitos ganhou popularidade considerável. Entretanto, a metodologia de diferenças finitas ainda permanece como uma técnica fundamental e muito utilizada devido sua eficácia, aplicabilidade e fácil implementação.

Observa-se na literatura que o método utilizado com mais frequência é o MDF explícito de primeira e segunda ordem, esse método, normalmente, envolve a discretização do domínio de interesse em uma malha estruturada e aproxima-se as derivadas por diferenças finitas. Sua implementação é relativamente simples, por exemplo, para aproximar o valor da primeira derivada de uma função $\phi$, podemos usar uma fórmula de diferenças finitas centrada dada por $\phi^{\prime}=\frac{\phi_{i+1}-\phi_{i-1}}{2 h}, i=0, \pm 1, \pm 2, \ldots$, onde $h$ é tamanho do passo de uma partição do domínio de $\phi$. Nesse método, a aproximação dos termos envolvendo derivadas da função é feita usando expansão em série de Taylor [9].

O erro local de truncamento, ou a medida pela qual a fórmula de diferenciação se aproxima da primeira derivada, é dado por $\tau_{i}=-\frac{1}{6} h^{2} \phi_{i+\epsilon}^{\prime \prime \prime}$, onde $\epsilon \in(i, i+1)$, [9]. Esse erro de truncamento se aproxima de zero quando a taxa $h^{2}$ se aproxima de zero, ou seja, quando $h \rightarrow 0$. Além disso, dizemos que o erro de truncamento é de ordem $h^{2}$, que denota-se por $O\left(h^{2}\right)$.

Para aproximar a solução de uma equação diferencial o processo é análogo, em cada ponto da malha, o valor da função (ou funções) desejada é tratada como incógnita, e a equação diferencial governante é aproximada por um sistema de equações algébricas que pode ser resolvido por um método de solução apropriado.

A precisão do método depende da aproximação dos operadores de diferenças finitas utilizados e da discretização da malha. Uma malha muito refinada aumenta a precisão da solução numérica mas também aumenta, relativamente, a dimensão do problema a ser resolvido.

Segundo [42] a desvantagem do MDF é exigir uma malha estruturada, ao contrário do método de elementos finitos que são projetados para acomodar malhas altamente desestruturadas, bem como dados e soluções de problemas irregulares, pois subdivide o domínio de um problema em partes menores, denominadas elementos finitos. Por outro lado, o MDF se adequa mais facilmente à análise de erros de truncamento obtido na expansão em série de Taylor, e esta propriedade será explorada no presente trabalho para desenvolver métodos de aproximações de alta ordem. 
A análise de erros de truncamento na expansão em série de Taylor para aproximações de diferenças finitas centrais mostra que o esquema aproxima os valores das derivadas com $O\left(h^{2}\right)$ à medida que o espaçamento $h$, entre os pontos da malha, se aproxima de zero.

Métodos com precisão melhor do que $O\left(h^{2}\right)$ são chamados métodos de alta ordem. Estes métodos são desejáveis porque a sua implementação permite malhas mais grossas com resultados precisos, diminuindo assim o custo computacional. O procedimento padrão para alcançar precisão de ordem superior é aumentar o stencil nas aproximações das derivadas. Em geral, em uma malha uniforme, uma derivada de ordem $p$, se $p$ é par, pode ser aproximado com ordem $O\left(h^{m}\right)$ com $p+m-1$ pontos. Se $p$ é ímpar, são necessários $p+m$ pontos.

Para derivadas de alta ordem, a aproximação exige pontos adicionais na malha e isso aumenta a complexidade do algoritmo. Em geral, as derivadas de ordem $p$, se $p$ é par, podem ser aproximados pelo MDF com $p+1$ pontos simetricos localizados na malha. Os coeficientes da fórmula de aproximação para este caso são simétricos.

Para $p$ ímpar, $p+2$ nós são necessários na malha e os coeficientes, neste caso, são antisimétricos, isto significa que o coeficiente central para a fórmula de aproximação é sempre zero [42].

Uma teoria geral para diferenças finitas foi desenvolvida por [35] para aproximação de primeira e segunda derivadas até ordem de $O\left(h^{n-1}\right)$ em uma malha com $n$ nós. Os métodos de alta ordem obtidos desta maneira exigem sempre stencils que utilizam pontos de malha localizados além dos diretamente ligados ao ponto central sobre o qual a derivada está sendo aproximada. Isto complica formulações perto da fronteira, aumenta a largura de banda da matriz e aumenta os requisitos de comunicação e implementação em arquiteturas de programação paralela.

Nas últimas décadas, muitos pesquisadores examinaram um grande número de fórmulas de diferenças finitas e descobriram que embora as aproximações tenham precisão de ordem verificada, elas podem apresentar oscilações ou instabilidade computacional (ver Gupta (1991) [15]; Li et al. (1995) [30]; Mancera (2006) [32]), o que desencadeou um grande interesse em aprofundar uma pesquisa em torno dos métodos compactos.

Como mencionado acima, fórmulas de diferenças finitas compactas são conhecidas há quase cinquenta anos. Algumas fórmulas são relatados por Collatz, [8]. No entanto, a sua implementação começou no início de 1970 para alguns problemas de mecânica dos fluidos [1], [18], [43]. Desde então, várias classes distintas de esquemas compactos foram desenvolvidas.

Hirsch, [18] apresentou comparações entre métodos compactos e não compactos. Nesta análise, uma aproximação não compacta de $2^{a}$ ordem e uma aproximação compacta de $4^{a}$ ordem foram avaliadas em relação à precisão numérica. Foi realizado um teste computacional simulando a utilização das duas aproximações.

Com base nesta investigação, Hirsch concluiu que para trabalhar com o método de $2^{a}$ ordem não compacto, é necessário gerar uma malha computacional quatro vezes mais refinada que a malha utilizada na simulação com a aproximação de $4^{a}$ ordem compacta para gerar resultados com precisão semelhantes. Além disso, os resultados mostram que o custo computacional é efetivamente reduzido em até 20 vezes. Hirsh também confirma através da comparação de aproximações de mesma ordem de precisão que os métodos compactos possuem um erro de truncamento menor que os métodos não compactos.

Em 1991, Lele [26] publicou um artigo com uma análise aprofundada de esquemas compactos. Ele sugere o uso de métodos compactos de alta ordem e apresenta esquemas para a primeira e segunda derivada de $2^{a}$ até $10^{a}$ ordem. Lele também propõe esquemas para serem aplicados nos pontos da fronteira e próximos às fronteiras. Estes métodos compactos são resolvidos de forma implícita, isto é, as derivadas espaciais discretizadas geram um sistema n-diagonal de equações lineares que pode ser resolvido calculando a inversa da matriz dos 
coeficientes relacionado ao sistema.

Gupta, [16] considerou a equação de convecção-difusão com coeficientes variáveis, esta equação muitas vezes aparece na descrição de fenômenos de transporte. Quando tal equação é discretizada usando diferenças centrais, o esquema resultante tem um erro de truncamento de ordem $O\left(h^{2}\right)$. Neste caso, os métodos iterativos para a solução do sistema resultante de equações lineares não converge quando os termos convectivos dominam. Uma alternativa para adequar a solução é usar aproximações progressivas ou regressivas para os termos convectivos, porém a ordem do erro de truncamento cai para $O(h)$, portanto isso requer um refinamento de malha que computacionalmente é mais custoso. Diante disso, Gupta desenvolveu um novo esquema de diferenças finitas para a equação de convecção-difusão com coeficientes variáveis. O esquema é definido em uma única célula quadrada de tamanho $2 h$ ao longo de um estêncil de 9 pontos com um erro de truncamento de $O\left(h^{4}\right)$, porém o método só pode ser aplicado em malhas uniformes. O sistema resultante de equações foi resolvido por métodos iterativos.

Tendo como motivação os resultados apresentados por Young e Dauwalder [45], que desenvolveram um esquema compacto de $O\left(h^{4}\right)$ com stencil de 9 pontos, Ananthakrishnaiah, Manohar e Stephenson [3] desenvolveram uma abordagem simples para resolver um problema elíptico bidimensional com coeficientes variáveis. Foi desenvolvido um esquema de diferenças finitas compacta em uma malha quadrada uniforme, obtendo um método de quarta ordem de treze pontos adaptado para pontos perto da fronteira.

Métodos de diferenças finitas de alta ordem do tipo compacto também são aplicados na solução das equações de Navier-Stokes e são computacionalmente eficientes e estáveis e as soluções numéricas obtidas são altamente precisas, [15], [30]. Esses métodos têm como atrativo molécula computacional pequena utilizando os oito pontos vizinhos mais próximos do ponto central dessa molécula, fácil implementação e resultados mais precisos mesmo quando usado malha grosseira, enquanto os métodos do tipo não compacto resultam em moléculas computacionais enormes.

Dando continuidade à pesquisa, Gupta desenvolveu aproximações de diferenças finitas de alta precisão para equações diferenciais elíptica, dando ênfase na equação de convecçãodifusão. Trata-se de aproximações compacta, com um erro de truncamento de quarta ordem. Neste artigo, Gupta adaptou essas aproximações de alta precisão na solução das equações de Navier-Stokes. Para verificação da metodologia, foi resolvido o problema modelo da cavidade controlada por baixo número de Reynolds, depois os resultados foram comparados com as soluções disponíveis na literatura e resultados obtidos por outros autores. Em todos os testes observou-se que o método compacto produziu resultados de alta precisão mesmo usando malha grosseira. Gupta conclui destacando que o método tem potencial para uso na solução de problemas importantes de fluxo de fluido viscoso, [15].

Zhang, [14] também contribuiu para a teoria dos métodos compactos com o esquema de diferença finita compacta de quarta ordem, este método foi utilizado para resolver as equações de difusão-convecção bidimensional influenciada pela camada limite. O domínio é discretizado em uma malha não uniforme. Uma técnica de transformação de coordenada é utilizada para mapear a malha não uniforme e transformá-la em uma malha uniforme, na qual os esquemas de diferenças são aplicados. Um método multigrid e uma técnica de pré-condicionamento multinível são usados para resolver os sistemas lineares dispersos resultantes.

Mancera, [32], resolveu o mesmo problema apresentado por [31], que foi proposto inicialmente por [16] usando um método compacto de quarta ordem, onde as velocidades são tratados separadamente. Considerou-se um procedimento para obter um método compacto de quarta ordem aplicado na solução das equações de Navier-Stokes utilizando o programa de 
manipulação algébrica Maple. Vale ainda ressaltar que Mancera e Hunt exibem um processo de construção do método compacto que se baseia em [15] e [30].

O procedimento empregado para testar o método consiste na resolução de um problema com solução analítica e outros seis do tipo cavidade. Mancera e Hunt estenderam o procedimento mencionado anteriormente para malhas não uniforme e resolveram o problema do degrau com face para frente. Também foi utilizado diferenças centrais de segunda ordem para moderar os números de Reynolds.

Mais recentemente Karaa, [20], propos um esquema de diferença finita de quarta ordem de 9 pontos para problemas elípticos $2 \mathrm{D}$ com derivada mista e coeficientes variáveis. A mesma abordagem foi estendida para derivar uma classe de esquemas compactos de dois níveis de alta ordem com discretização do tempo ponderado para resolver problemas parabólicos 2D com uma derivada mista. Os esquemas são de quarta ordem de precisão no espaço e segunda ou menor ordem de precisão no tempo, dependendo da escolha de um parâmetro médio ponderado $\mu$. A estabilidade incondicional é provada para $0,5 \leq \mu \leq 1$, também são apresentadas soluções numéricas que confirmam a análise teórica e a precisão de alta ordem dos esquemas.

Baseado nos trabalhos já mencionados, apresenta-se neste trabalho um estudo detalhado de métodos compactos aplicado na solução de equações diferenciais parciais elípticas. A equação de Poisson assume posição de destaque neste trabalho, pois para resolver as equações de Navier-Stokes para escoamentos bifásicos com o Método da Projeção de Chorin-Temam, [7], também passamos pela resolução de uma equação elíptica para a correção da pressão, como foi discutido no trabalho [2] e [44].

\subsection{Objetivos}

De maneira geral, o objetivo deste trabalho é fazer um estudo detalhado e o desenvolvimento de uma metodologia numérica compacta, aplicáveis na resolução eficiente de problemas elípticos com coeficientes variáveis.

No contexto da motivação científica, fluidos newtonianos e não newtonianos em escoamentos incompressíveis estacionários são gerados por equações elípticas e parabólicas, com a função incógnita localizada em diferentes posições das células computacionais que formam a discretização do domínio computacional, por exemplo: centros de células e meios de arestas. Desta forma, o ponto de partida e a ferramenta principal desse trabalho foi o código Hig-Tree em desenvolvimento no Instituto de Ciências Matemáticas e Computação (ICMC), USP São Carlos detalhado em [23], o código representa uma estrutura de dados como uma árvore em que cada nível corresponde a um refinamento da malha. Assim temos a autonomia de definir e discretizar o domínio computacional por malhas não estruturadas.

Como citado acima, a estrutura de dados representado pela Hig-Tree agrega uma infinidade de possibilidades de refinamento de malha, nesse sentido a discretização dos problemas que serão resolvidos resultará em um sistema de equações lineares que não podem ser resolvidas empregando um único método de solução e necessita da paralelização de métodos, iterativos e diretos, de solução. Por esta razão a biblioteca PETSc tornou-se uma nova ferramenta de estudo desta dissertação.

Os objetivos específicos são:

- Entender o funcionamento das rotinas que compõem o cógido Hig-Tree, a fim de alterálo de maneira a permitir a implementação de outros métodos numéricos;

- Desenvolver detalhadamente uma metodologia numérica compacta de quarta ordem aplicável à problemas elípticos com coeficientes variáveis; 
- Implementar o método de diferenças finitas explícito de quarta ordem clássico, com intuito de comparar com o método compacto desenvolvido.

E, com os resultados obtidos, proporcionar uma referência que possa contribuir para trabalhos científicos na área. 


\section{Capítulo 2}

\section{Estrutura HiGTree}

Este capítulo descreve os métodos utilizados para resolução numérica de equações diferenciais parciais em uma malha hierárquica do tipo "HiG-tree". São descritos detalhes sobre este tipo de malha, bem como os métodos utilizados para discretização por diferenças finitas generalizadas e interpolações necessárias entre os diferentes níveis de refinamento. Finalmente são apresentados os mecanismos necessários para a resolução de uma equação de Poisson em um domínio genérico.

\subsection{Malhas hierárquicas}

Neste trabalho, foi usada uma estrutura de dados chamada Hig-Tree em desenvolvimento no Instituto de Ciências Matemáticas e Computação, USP São Carlos detalhado em [23], que representa uma árvore em que cada nível corresponde a um refinamento da malha, alguns exemplos clássicos deste tipo de estrutura são as malhas octree (três dimensões) e quadtree (duas dimensões). A estrutura usada permite que haja refinamentos arbitrários em qualquer nível da árvore, além da possibilidade de refinamento adaptativo como pode ser visto na figura 2.1 que é um exemplo de malha deslocada em duas dimensões em que nem todas as células tem o mesmo tamanho. A figura 2.2 ilustra o esquema em duas dimensões para uma malha com diversos níveis de refinamento, a extensão para três dimensões é natural uma vez que a estrutura é suficientemente genérica para isso.

\subsection{Diferenças finitas generalizadas}

O método adotado para discretização de equações diferenciais é o método de diferenças finitas. Tal método é baseado na expansão em série de Taylor da função a ser aproximada e de suas derivadas, cuja omissão de termos de alta ordem desta série resulta em fórmulas adequadas para as aproximações.

Um exemplo clássico é a discretização de uma equação de Poisson do tipo

$$
\nabla^{2} u=f
$$

com condições de contorno adequadas. Simplificando o domínio por um retângulo em um espaço de dimensão dois $\Omega=[a, b] \times[c, d]$, e utilizando uma malha cartesiana regular (do tipo grid) com espaçamentos $\delta x$ e $\delta y$, obtém-se a clássica fórmula do laplaciano de 5 pontos, dada por

$$
\frac{u_{i+1, j}-2 u_{i, j}+u_{i-1, j}}{\delta x^{2}}+\frac{u_{i, j+1}-2 u_{i, j}+u_{i, j-1}}{\delta y^{2}}+\mathcal{O}\left(\delta x^{2}+\delta y^{2}\right)=f_{i, j}
$$




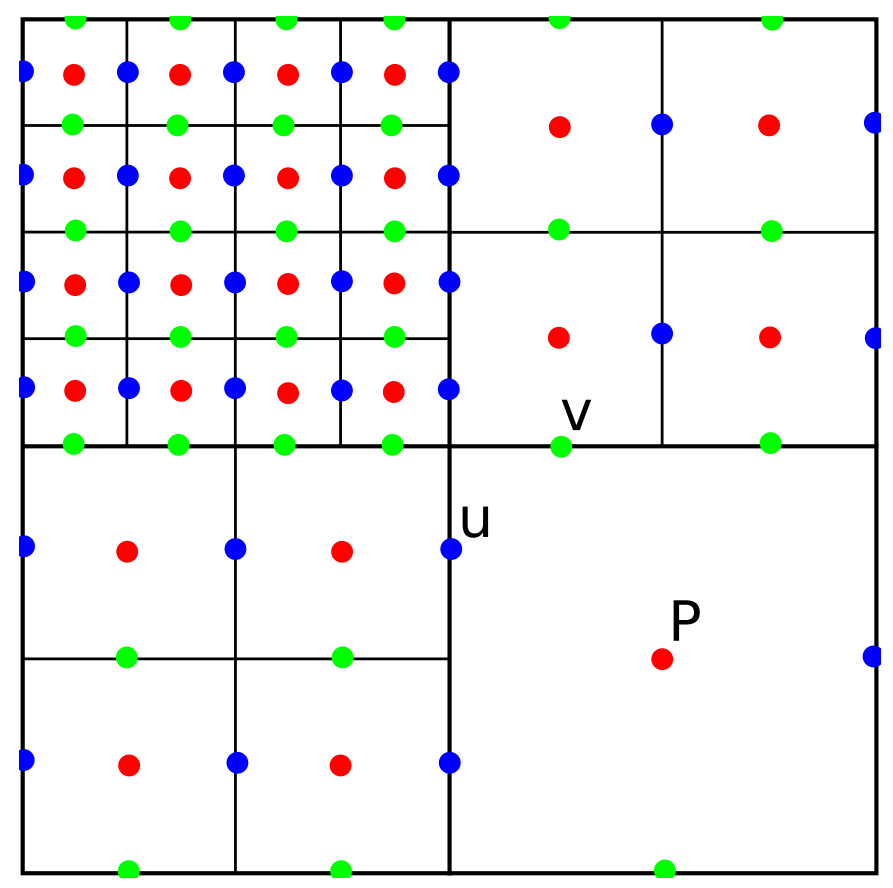

Figura 2.1: Malha deslocada.
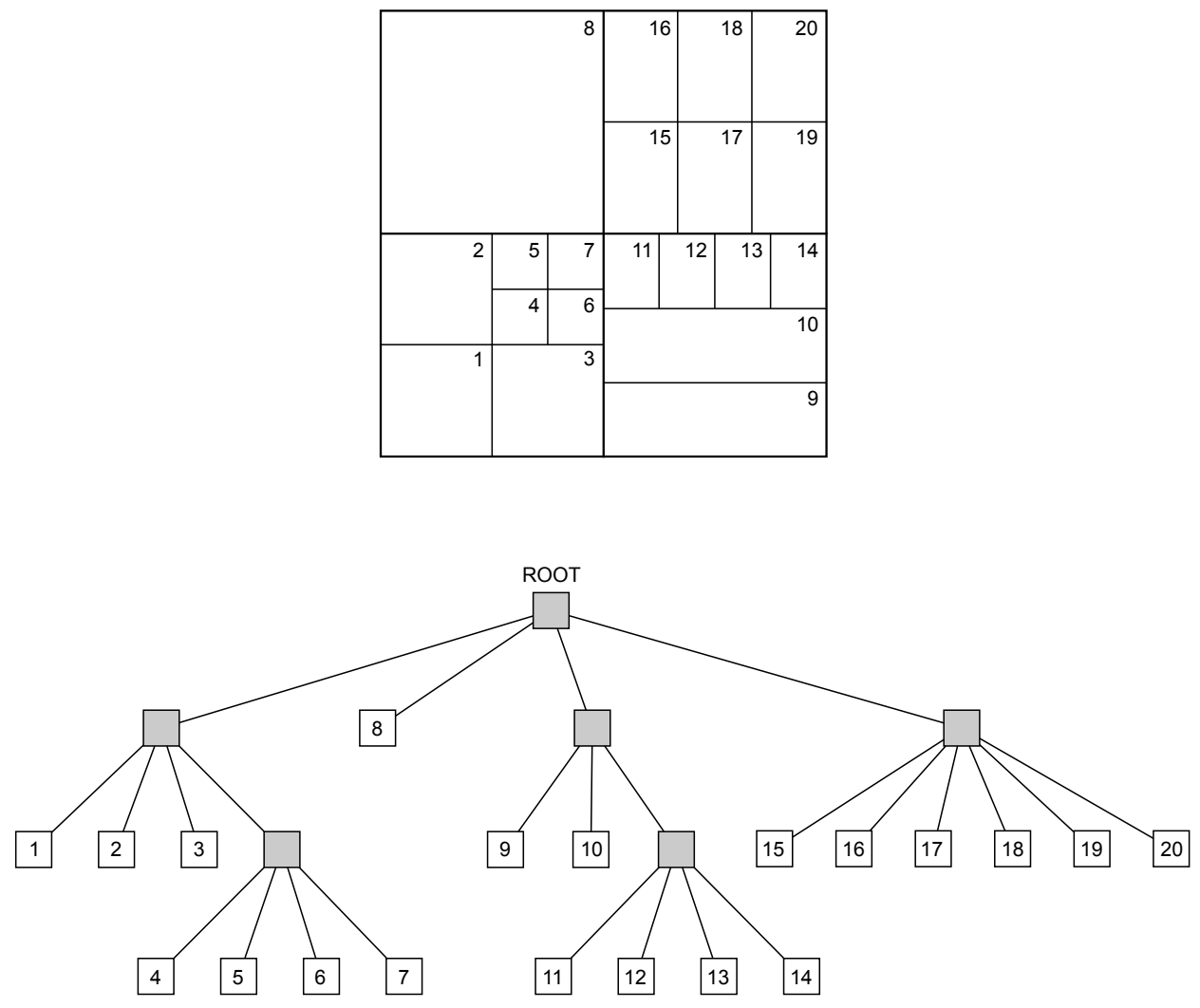

Figura 2.2: Exemplo de uma malha tipo HiG e sua representação na forma de árvore (HiG-Tree). 
ou ainda, desprezando-se os termos de ordem 2,

$$
\frac{U_{i+1, j}-2 U_{i, j}+U_{i-1, j}}{\delta x^{2}}+\frac{U_{i, j+1}-2 U_{i, j}+U_{i, j-1}}{\delta y^{2}}=f_{i, j}
$$

onde $U_{i, j}$ representa uma aproximação para a função $u_{i, j}$, avaliada em $\left(x_{i}, y_{j}\right)=(a+i \delta x, b+$ $j \delta y)$, e $f_{i, j}=f\left(x_{i}, y_{j}\right)$. Com esta fórmula, mais as condições de contorno, é possível montar um sistema da forma

$$
L \mathbf{u}=\mathbf{f},
$$

cuja a solução é o vetor de aproximações $\mathbf{u}=\left(U_{1,1}, U_{1,2}, \ldots\right)$ para a função $u$ original, avaliada nos vértices da malha cartesiana.

O objetivo é utilizar diferenças finitas em uma malha cartesiana irregular, então tal aproximação deve ser modificada, pois uma discretização por um laplaciano de 5 pontos resultaria o envolvimento de valores não conhecidos no interior da malha. Desta forma é preciso realizar um procedimento de interpolação que seja genérico o suficiente para englobar qualquer configuração de células (como no exemplo da figura 2.2), e que seja preciso o suficiente para não deteriorar a ordem de convergência do método numérico. Uma clara desvantagem da utilização de malhas irregulares como as exemplificadas anteriormente é que uma numeração do tipo matricial $(i, j)$ não é mais possível, sendo necessária a definição de uma enumeração dos nós válidos da malha. Tal enumeração é fornecida pela estrutura HiG-Tree, contudo, diferentes formas de enumeração podem ser implementadas, com impacto direto na estrutura da matriz do sistema resultante, e consequentemente no desempenho dos métodos numéricos utilizados para resolvê-lo.

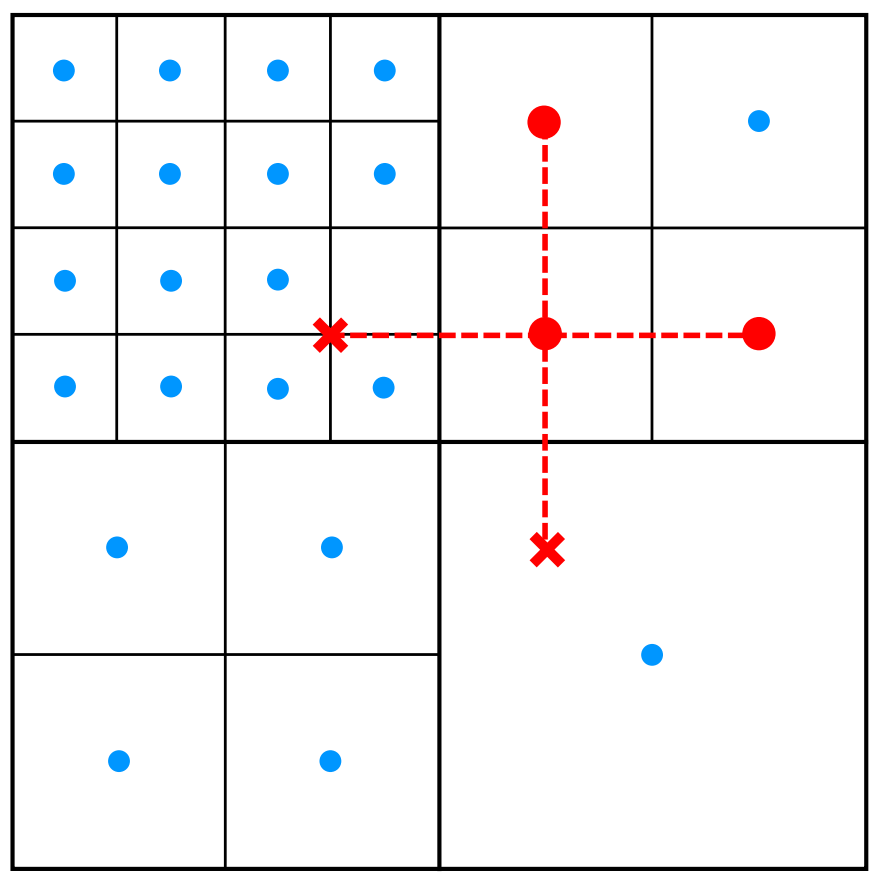

Figura 2.3: Exemplo de um estêncil resultando em pontos a serem interpolados ( $x_{L}$ e $\left.x_{B}\right)$.

Considere o caso ilustrado na figura 2.3 , onde um estêncil de cinco pontos é utilizado para discretizar as derivadas no ponto $x_{C}$. É fácil notar que, devido a configuração irregular da malha, não existe nó da malha que armazene um valor de $u$ para os pontos $x_{L}$ e $x_{B}$, ou seja, não existem incógnitas $U_{L}$ e $U_{B}$ para o sistema linear. Neste exemplo, é necessário obter então uma interpolação dos nós vizinhos, pertencentes à malha, mais próximos às incógnitas 
não existentes. Digamos que uma interpolação adequada resulte na seguinte expressão para $U_{L}$

$$
U_{L} \approx w_{1} U_{j_{1}}+w_{2} U_{j_{2}}+\cdots+w_{n} U_{j_{n}}
$$

sendo $U_{j_{1}}, U_{j_{2}}, \ldots, U_{j_{n}}$ valores conhecidos na malha. Escrevendo a fórmula usual do laplaciano de 5 pontos para o ponto $x_{C}$, obtém-se uma equação para $U_{C}$, a saber,

$$
\frac{U_{L}-2 U_{C}+U_{R}}{\delta x^{2}}+\frac{U_{T}-2 U_{C}+U_{B}}{\delta y^{2}}=f_{C},
$$

que pode ainda ser reescrita como

$$
a_{L} U_{L}+a_{R} U_{R}+a_{T} U_{T}+a_{B} U_{B}+a_{C} U_{C}=f_{C},
$$

onde

$$
a_{L}=\frac{1}{\delta x^{2}}, \quad a_{R}=\frac{1}{\delta x^{2}}, \quad a_{T}=\frac{1}{\delta y^{2}}, \quad a_{B}=\frac{1}{\delta y^{2}}, \quad a_{C}=-\frac{2}{\delta x^{2}}-\frac{2}{\delta y^{2}} .
$$

Substituindo o valor interpolado de $U_{L}$ na equação, obtém-se

$$
a_{L}\left(w_{1} U_{j_{1}}+w_{2} U_{j_{2}}+\cdots+w_{n} U_{j_{n}}\right)+a_{R} U_{R}+a_{T} U_{T}+a_{B} U_{B}+a_{C} U_{C}=f_{C},
$$

gerando-se novos coeficientes e uma equação. Note que o mesmo deve ser feito para $U_{B}$, para que a equação envolva finalmente somente valores conhecidos da malha. Note que este processo pode ser repetido arbitrariamente para qualquer valor que ainda não esteja definido na malha, e que incógnitas repetidas podem ser agrupadas em um único coeficiente multiplicativo. Desta forma, obtém-se uma expressão que é uma combinação linear formada somente por incógnitas verdadeiras, para valores definidos na malha, que define a equação para a incógnita $U_{C}$ no sistema linear.

A próxima seção explica como obter uma interpolação que seja suficientemente genérica e precisa, e como obter os pesos $w_{j_{1}}, w_{j_{2}}, \ldots, w_{j_{n}}$ necessários.

\subsection{Interpolações}

\subsubsection{Aproximação polinomial}

Um dos objetivos do método descrito aqui é obter uma aproximação para o valor de uma propriedade em um dado ponto $(x, y, z)$ em função dos valores da propriedade em $m$ outros pontos $\left(x_{1}, y_{1}, z_{1}\right), \ldots,\left(x_{m}, y_{m}, z_{m}\right)$. Os valores nos $m$ pontos não são conhecidos a priori. Denotamos o valor em um ponto $(x, y, z)$ por $P(x, y, z)$ um polinômio de grau no máximo $r$,

$$
P(x, y, z)=\alpha_{i_{1}, j_{1}, k_{1}} x^{i_{1}} y^{j_{1}} z^{k_{1}}+\cdots+\alpha_{i_{n}, j_{n}, k_{n}} x^{i_{n}} y^{j_{n}} z^{k_{n}}=c^{t} \alpha,
$$

onde $0 \leq i_{s}+j_{s}+k_{s} \leq r$, para $s=1, \ldots, n$. Observe que poderia ser uma combinação de funções não polinomiais.

Assim, o método deve obter os coeficientes $w_{i}$, para $1 \leq i \leq m$, de forma que

$$
P(x, y, z)=\sum_{i=1}^{m} w_{i} P\left(x_{i}, y_{i}, z_{i}\right)=w^{t} b .
$$

Assim, 


$$
\begin{gathered}
b=\left(\begin{array}{c}
P\left(x_{1}, y_{1}, z_{1}\right) \\
\vdots \\
P\left(x_{m}, y_{m}, z_{m}\right)
\end{array}\right)=\left(\begin{array}{ccc}
x_{1}^{i_{1}} y_{1}^{j_{1}} z_{1}^{k_{1}} & \cdots & x_{1}^{i_{n}} y_{1}^{j_{n}} z_{1}^{k_{n}} \\
\vdots & & \vdots \\
x_{m}^{i_{1}} y_{m}^{j_{1}} z_{m}^{k_{1}} & \cdots & x_{m}^{i_{n}} y_{m}^{j_{n}} z_{m}^{k_{n}}
\end{array}\right)\left(\begin{array}{c}
\alpha_{1} \\
\vdots \\
\alpha_{n}
\end{array}\right)= \\
=\left(\begin{array}{c}
a_{1}^{t} \\
\vdots \\
a_{m}^{t}
\end{array}\right)\left(\begin{array}{c}
\alpha_{1} \\
\vdots \\
\alpha_{n}
\end{array}\right)=A \alpha
\end{gathered}
$$

Logo,

$$
w^{t} b=w^{t} A \alpha=c^{t} \alpha
$$

e uma solução é dada por $w^{t} A=c^{t}$, ou

$$
A^{t} w=c .
$$

Se $m \geq n$ e $A$ tem posto máximo $n, w$ pode ser obtido pelo método dos mínimos quadrados.

\subsubsection{Aproximação pelo Método dos Mínimos Quadrados}

Fazendo a decomposição QR de $A$, temos:

$$
\begin{aligned}
A_{m \times n} & =\left(\begin{array}{l}
A_{1 n \times n} \\
A_{2 m-n \times n}
\end{array}\right)=Q_{m \times m} R_{m \times n}^{*} \\
& =Q_{m \times m}\left(\begin{array}{l}
R_{n \times n} \\
0_{m-n \times n}
\end{array}\right)=\left(\begin{array}{ll}
Q_{11} & Q_{12} \\
Q_{21} & Q_{22}
\end{array}\right)\left(\begin{array}{c}
R \\
0
\end{array}\right)
\end{aligned}
$$

Assim,

$$
\begin{gathered}
w=A^{+} c^{t}=A\left(A^{t} A\right)^{-1} c^{t}=Q R^{*}\left(R^{* t} Q^{t} Q R^{*}\right)^{-1} c^{t}=Q R^{*}\left(R^{t} R\right)^{-1} c^{t}= \\
=Q R^{*} R^{-1}\left(R^{t}\right)^{-1} c^{t}=Q\left(\begin{array}{l}
R \\
0
\end{array}\right) R^{-1}\left(R^{t}\right)^{-1} c^{t}=Q\left(\begin{array}{l}
\left(R^{t}\right)^{-1} \\
0
\end{array}\right) c^{t}
\end{gathered}
$$

Fazendo $u=Q^{t} w$ e $u=\left(\begin{array}{l}u_{1} \\ u_{2}\end{array}\right)$, temos:

$$
\begin{gathered}
u=Q^{t} w=\left(\begin{array}{l}
\left(R^{t}\right)^{-1} \\
0
\end{array}\right) c^{t} \\
u=\left(\begin{array}{l}
u_{1} \\
u_{2}
\end{array}\right)=\left(\begin{array}{l}
\left(R^{t}\right)^{-1} \\
0
\end{array}\right) c^{t} \\
\left\{\begin{array}{l}
u_{1}=\left(R^{t}\right)^{-1} c^{t} \\
u_{2}=
\end{array}\right.
\end{gathered}
$$

Daí, resolvendo o sistema triangular inferior $R^{t} u_{1}=c^{t}$, temos $w=Q u$, com $u_{2}=0$.

Resumindo, são dados $A$ e $c^{t}$ e queremos $w$, então são feitos três passos:

1. Fazer a decomposição $\mathrm{QR}$ de $A$, isto é, $A=Q R^{*}$;

2. Resolver o sistema triangular inferior $R^{t} u_{1}=c^{t}$;

3. Fazer $w=Q u \operatorname{com} u_{2}=0$. 
Observe que para o caso de $m=n$, a aproximação polinomial $P$ será o polinômio interpolador.

\subsubsection{Cálculo dos coeficientes}

Seja

$$
C=\left(\begin{array}{cc}
A_{1} & B_{1} \\
A_{2} & B_{2}
\end{array}\right)=\left(\begin{array}{ll}
Q_{11} & Q_{12} \\
Q_{21} & Q_{22}
\end{array}\right)\left(\begin{array}{cc}
R & 0 \\
0 & I
\end{array}\right)=\left(\begin{array}{ll}
Q_{11} R & Q_{12} \\
Q_{21} R & Q_{22}
\end{array}\right)
$$

Desde que,

$$
\begin{gathered}
I_{m}=Q^{t} Q=\left(\begin{array}{ll}
Q_{11}^{t} & Q_{21}^{t} \\
Q_{12}^{t} & Q_{22}^{t}
\end{array}\right)\left(\begin{array}{ll}
Q_{11} & Q_{12} \\
Q_{21} & Q_{22}
\end{array}\right)= \\
=\left(\begin{array}{cc}
Q_{11}^{t} Q_{11}+Q_{21}^{t} Q_{21} & Q_{11}^{t} Q_{12}+Q_{21}^{t} Q_{22} \\
Q_{12}^{t} Q_{11}+Q_{12}^{t} Q_{22} & Q_{12}^{t} Q_{12}+Q_{22}^{t} Q_{22}
\end{array}\right)=\left(\begin{array}{ll}
I_{n} & 0 \\
0 & I_{m-n}
\end{array}\right),
\end{gathered}
$$

Temos que,

$$
\begin{aligned}
& A^{t} B=\left(\begin{array}{ll}
A_{1}^{t} & A_{2}^{t}
\end{array}\right)\left(\begin{array}{l}
B_{1} \\
B_{2}
\end{array}\right)=A_{1}^{t} B_{1}+A_{2}^{t} B_{2}=R\left(Q_{11}^{t} Q_{12}+Q_{21}^{t} Q_{22}\right)=0, \\
& B^{t} B=\left(\begin{array}{ll}
B_{1}^{t} & B_{2}^{t}
\end{array}\right)\left(\begin{array}{c}
B_{1} \\
B_{2}
\end{array}\right)=B_{1}^{t} B_{1}+B_{2}^{t} B_{2}=Q_{12}^{t} Q_{12}+Q_{22}^{t} Q_{22}=I_{m-n},
\end{aligned}
$$

e

$$
C^{t} C=\left(\begin{array}{cc}
A_{1}^{t} & A_{2}^{t} \\
B_{1}^{t} & B_{2}^{t}
\end{array}\right)\left(\begin{array}{cc}
A_{1} & B_{1} \\
A_{2} & B_{2}
\end{array}\right)=\left(\begin{array}{cc}
R^{t} R & 0 \\
0 & I
\end{array}\right)
$$

Logo $\operatorname{Det}(C)= \pm \operatorname{Det}(R)=R_{1,1} \cdots R_{n, n}$, e se $A$ tem posto máximo $R_{i, i} \neq 0$ e

$$
C^{t} w=\left(\begin{array}{cc}
A_{1}^{t} & A_{2}^{t} \\
B_{1}^{t} & B_{2}^{t}
\end{array}\right) w=\left(\begin{array}{c}
c \\
0
\end{array}\right)
$$

tem solução única.

Se $m=n, C^{t}=A_{1}^{t}=\left(a_{1} \cdots a_{n}\right)$. Logo se $x=x_{i}(i=1, \ldots, n)$ tem-se $c=a_{i}$ e $C^{t} w=d$, segue que $A_{1} w=c$ que implica que

$$
\left(a_{1} \cdots a_{i} \cdots a_{n}\right) w=a_{i},
$$

tem solução $w_{k}=1$ se $i=k$ e $w_{k}=0$ se $i \neq k$.

Se $m>n$ e $x=x_{i}(i=1, \ldots, n)$,

$$
C^{t} w=\left(\begin{array}{cc}
A_{1}^{t} & A_{2}^{t} \\
B_{1}^{t} & B_{2}^{t}
\end{array}\right) w=\left(\begin{array}{ccccc}
a_{1} & \cdots & a_{i} & \cdots & a_{m} \\
b_{1} & \cdots & b_{i} & \cdots & b_{m}
\end{array}\right) w=\left(\begin{array}{c}
c \\
0
\end{array}\right)=\left(\begin{array}{c}
a_{i} \\
0
\end{array}\right)
$$

Como

$$
\begin{gathered}
C^{t}=\left(\begin{array}{cc}
A_{1}^{t} & A_{2}^{t} \\
B_{1}^{t} & B_{2}^{t}
\end{array}\right)=\left(\begin{array}{cc}
R^{t} & 0 \\
0 & I
\end{array}\right)\left(\begin{array}{ll}
Q_{11}^{t} & Q_{21}^{t} \\
Q_{12}^{t} & Q_{22}^{t}
\end{array}\right)=\left(\begin{array}{cc}
R^{t} & 0 \\
0 & I
\end{array}\right) Q^{t}, \\
\left(\begin{array}{cc}
A_{1}^{t} & A_{2}^{t} \\
0 & 0
\end{array}\right)=\left(\begin{array}{cc}
R^{t} & 0 \\
0 & 0
\end{array}\right)\left(\begin{array}{ll}
Q_{11}^{t} & Q_{21}^{t} \\
Q_{12}^{t} & Q_{22}^{t}
\end{array}\right)=\left(\begin{array}{cc}
R^{t} & 0 \\
0 & 0
\end{array}\right) Q^{t},
\end{gathered}
$$

que implica que 


$$
\left(\begin{array}{c}
a_{i} \\
0
\end{array}\right)=\left(\begin{array}{cc}
A_{1}^{t} & A_{2}^{t} \\
0 & 0
\end{array}\right) e_{i}=\left(\begin{array}{cc}
R^{t} & 0 \\
0 & 0
\end{array}\right) Q^{t} e_{i}
$$

Logo,

$$
\begin{gathered}
C^{t} w=d \\
\left(\begin{array}{cc}
R^{t} & 0 \\
0 & I
\end{array}\right) Q^{t} w=\left(\begin{array}{cc}
R^{t} & 0 \\
0 & 0
\end{array}\right) Q^{t} e_{i} . \\
w=Q\left(\begin{array}{cc}
R^{-t} & 0 \\
0 & I
\end{array}\right)\left(\begin{array}{cc}
R^{t} & 0 \\
0 & 0
\end{array}\right) Q^{t} e_{i} . \\
w=Q\left(\begin{array}{ll}
I & 0 \\
0 & 0
\end{array}\right) Q^{t} e_{i} .
\end{gathered}
$$

\subsubsection{Diferenças Finitas Generalizadas}

Considere a equação

$$
f^{\prime \prime}(x)=g(x)
$$

em um intervalo $[a, b] \operatorname{com} f(a)=\alpha$ e $f(b)=\beta$.

Se subdividirmos o intervalo $[a, b]$ em intervalos igualmente espaçados $x_{i}=x_{0}+i h, x_{0}=a$ e $x_{n}=b$, uma discretização para a equação acima utilizando diferenças finitas é dada por:

$$
\frac{f_{i-1}-2 f_{i}+f_{i+1}}{h^{2}}=g_{i}
$$

este método é um método com erro local de truncamento da ordem de $h^{2}$, isto é,

$$
\frac{f_{i-1}-2 f_{i}+f_{i+1}}{h^{2}}-g_{i}=f^{\prime \prime}\left(x_{i}\right)-g\left(x_{i}\right)+O\left(h^{2}\right)
$$

Caso o espaçamento dos pontos não seja uniforme, podemos o método dos mínimos quadrados para aproximar $f_{i-1}^{*} \sim f\left(x_{i}-h\right)$ e $f_{i+1}^{*} \sim f\left(x_{i}+h\right)$, isto é,

$$
\begin{aligned}
& f_{i-1}^{*}=\sum_{x_{j} \in V\left(x_{i}-h\right)} w_{j} f_{j} \\
& f_{i+1}^{*}=\sum_{x_{k} \in V\left(x_{i}+h\right)} w_{k} f_{k}
\end{aligned}
$$

Se tomarmos os pontos na vizinhança em número suficiente, temos que $f_{i-1}^{*}=f\left(x_{i}-\right.$ $h)+O\left(h^{4}\right)$ e $f_{i+1}^{*}=f\left(x_{i}+h\right)+O\left(h^{4}\right)$.

Assim temos que:

$$
\begin{gathered}
\frac{f_{i-1}^{*}-2 f_{i}+f_{i+1}^{*}}{h^{2}}-g_{i}=\frac{f\left(x_{i}-h\right)+O\left(h^{4}\right)-2 f\left(x_{i}\right)+f\left(x_{i}+h\right)+O\left(h^{4}\right)}{h^{2}}-g_{i}= \\
=\frac{f\left(x_{i}-h\right)-2 f\left(x_{i}\right)+f\left(x_{i}+h\right)}{h^{2}}-g\left(x_{i}\right)+O\left(h^{2}\right)=f^{\prime \prime}\left(x_{i}\right)-g\left(x_{i}\right)+O\left(h^{2}\right)
\end{gathered}
$$

Desta forma o método fica:

$$
f_{i-1}^{*}-2 f_{i}+f_{i+1}^{*}=h^{2} g_{i}
$$




$$
\left(\sum_{x_{j} \in V\left(x_{i}-h\right)} w_{j} f_{j}\right)-2 f_{i}+\left(\sum_{x_{k} \in V\left(x_{i}+h\right)} w_{k} f_{k}\right)=h^{2} g_{i}
$$




\section{Capítulo 3}

\section{Metodologia numérica}

O Esquema Compacto de Diferenças Finitas (ECDF) é um método de resolução de equações diferenciais, que se baseia na aproximaçao de derivadas por aproximações de diferenças finitas implícitas representados por um estêncil pequeno. Neste capítulo, será tratado a dedução das fórmulas que compõem o ECDF. As fórmulas serão aplicadas no próximo capítulo.

\subsection{Modelagem Matemática}

Dinâmica de fluidos computacional é um dos campos da ciência que tem como objetivo descrever o comportamento dos fluidos (gases ou líquidos) e das leis que regem este comportamento através da obtenção de soluções numéricas para um sistema de equações diferenciais [30]. Os fluidos são geralmente classificados em compressíveis e incompressíveis, sendo que o primeiro sofre variações na densidade, enquanto no segundo a densidade permanece constante. Um aspecto relevante de um fluido é a sua viscosidade que pode ser interpretada como uma medida da resistência ao cisalhamento pelo fluido [34]. Com base nessa resistência ao cisalhamento, pode-se classificar os fluidos em newtonianos, em que a tensão de cisalhamento é diretamente proporcional a taxa de deformação, e os não newtonianos que não obedecem esse comportamento. Qualquer escoamento de um fluido é governado pelas equações de Navier-Stokes, as quais constituem um sistema de equações diferenciais parciais que descrevem o escoamento de fluidos compressíveis ou incompressíveis, turbulentos e laminares. Em particular, estas equações modelam alguns problemas de dinâmica dos fluidos como o fluxo sanguíneo através de artérias, veias e o movimento de agua e rios.

\subsection{Equações Diferencias Parciais}

Muitos fenômenos físicos são modelados por Equacões Diferenciais Parciais (EDPs). As grandezas físicas procuradas, como, por exemplo, velocidade e pressão de um fluido, normalmente, são representadas por modelos que dependem de muitas variáveis [9]. Para efeito de classificação, divide-se as EDPs de segunda ordem em três categorias básicas:

1. Elípticas (normalmente associadas com problemas estacionários). Um exemplo de equação elíptica é dado por

$$
\frac{\partial^{2} \varphi}{\partial x^{2}}+\frac{\partial^{2} \varphi}{\partial y^{2}}=0
$$

que é a equação de Laplace. 
2. Parabólicas (associadas com problemas de fluxo contendo mecanismos de dissipação). Um exemplo de equação parabólica é a equação do calor, dada por:

$$
\frac{\partial T}{\partial t}=\alpha \frac{\partial^{2} T}{\partial x^{2}}
$$

em que $T$ é a temperatura e $\alpha$ é o coeficiente de difusividade térmica do material.

3. Hiperbólicas (em geral associadas com problemas de fluxo que não contenham mecanismos de dissipação). Um exemplo de equação hiperbólica é dado por

$$
\frac{\partial^{2} \varphi}{\partial t^{2}}=\alpha^{2} \frac{\partial^{2} \varphi}{\partial x^{2}}
$$

que é a equação da onda, em que a constante $\alpha$ é a velocidade de propagação da onda.

Em geral EDPs podem ser relacionadas com problemas de fluxo de fluido, sendo as equações de Navier-Stokes o exemplo mais importante.

As equações de Navier-Stokes para um escoamento incompressível são dadas por:

$$
\begin{gathered}
\rho\left(\frac{\partial \mathbf{u}}{\partial t}+\mathbf{u} \cdot \nabla \mathbf{u}\right)=-\nabla p_{\nabla} \cdot(2 \mu \mathbf{d})+\mathbf{f} \\
\nabla \cdot \mathbf{u}=0
\end{gathered}
$$

onde $\rho$ é a densidade de massa (massa por unidade de volume), $\mathbf{d}=\frac{\nabla \mathbf{u}+\nabla \mathbf{u}^{T}}{2}$ é o tensor taxa de defomação de um fluido, $\mu$ é a viscosidade do fluido e $p$ é a pressão estática.

A condição inicial é dada por: $\mathbf{u}(x, 0)=\mathbf{u}_{0}$, e a condição de contorno para o campo de velocidade pode ser, entre outras, do tipo Dirichlet ou Neumann.

De acordo com [39] tentar desenvolver um esquema numérico para resolver as equações (3.4)-(3.5) significa lidar com algumas dificuldades destas equações: a não linearidade das equações devido ao termo convectivo, a falta de uma equação para a pressão, etc. A maioria dos esquemas partem de equações discretizadas no tempo. A seguir, apresentam-se algumas aproximações no tempo das equações de Navier-Stokes:

$$
\begin{cases}\rho\left(\frac{\mathbf{u}^{n+1}-\mathbf{u}^{n}}{\Delta t}+(\mathbf{u} \cdot \nabla \mathbf{u})^{n}\right) & =-\nabla p^{n+1}+\nabla \cdot[2 \mu \mathbf{d}]^{n}+\mathbf{f}^{n}, \\ \nabla \cdot \mathbf{u}^{n+1} & =0,\end{cases}
$$

O sistema (3.6) pode ser obtido integrando-se as equações de Navier-Stokes no intervalo $\left[t^{n}, t^{n+1}\right]\left(\Delta t=t^{n+1}-t^{n}\right)$ [21]. O esquema (3.6) é de primeira ordem no tempo. Uma solução direta do sistema acoplado não é praticável devido à necessidade de métodos robustos para lidar com sistemas não lineares de equações e ao custo computacional que isto acarreta.

As metodologias mais populares estão baseadas no desacoplamento do cálculo da velocidade e da pressão, que permite o uso de técnicas mais eficientes para cada um dos processos resultantes [39] [25].

Harlow e Welch [17] apresentaram o método MAC (marker and cell) desenvolvido para o tratamento de escoamentos incompressíveis com superfícies livres. A proposta para resolver as equações de Navier-Stokes consiste na geração de uma equação de Poisson para a pressão, a partir da equação do balanço da quantidade de movimento discreta no tempo e da condição de livre divergência da velocidade. Uma vez obtida a pressão, o campo de velocidade é calculado da equação de quantidade de movimento considerando a pressão calculada. Vários trabalhos foram desenvolvidos seguindo esta filosofia. Liu [11] usou esta técnica para cada 
sub-estágio do método de Runge-Kutta de quarta ordem empregado no estudo de esquemas de diferenças finitas usando a formulação vorticidade. Nos anos 60, Chorin [7] observou que para escoamentos incompressíveis, a pressão não tem um papel termodinâmico, ela tem por função forçar a condição de incompressibildade para o fluido. Esta observação motivou um esquema de discretização baseado na separação de operadores e é conhecido como o método de projeção ou o método de passo fracionário. Neste método, os cálculos da velocidade e da pressão são realizados por intermédio de dois passos: um preditor e outro corretor. No primeiro passo, um campo de velocidade auxiliar é calculado, resolvendo-se a equação da quantidade de movimento sem considerar o termo da pressão e a condição de incompressibilidade. No segundo passo, de acordo com o teorema de decomposição de Hodge [6] a velocidade auxiliar é projetada no espaço dos campos vetoriais com divergente zero para determinar a pressão (ou correção de pressão) e a velocidade correta (que satisfaz a condição de incompressibilidade).

\subsection{Equação de Poisson no Método de Projeção}

Como foi descrito da seção anterior, o método de projeção está baseado no desacoplamento do cálculo da velocidade e da pressão. Várias propostas foram apresentadas [4], [7], [22], [19] e as principais diferenças entre elas residem nas expressões para o cálculo da velocidade auxiliar e para o passo de projeção. Exemplos da aplicabilidade dos métodos de projeção em problemas práticos podem ser encontrados em [12], [13], [38], [33], [36], [37], [10]. A seguir mostraremos a equação elíptica que aparece no Método de Projeção. Para isto, usaremos um método de projeção explícito.

\subsubsection{Um método explícito de primeira ordem no tempo}

O método explícito a ser apresentado está baseado na aproximação de primeira ordem no tempo, (3.4), para as equações de Navier-Stokes (3.6). Estas aproximações serão resolvidas através do método de projeção, que será dividido em três passos:

\section{Passo 1:}

Cálculo de uma velocidade auxiliar, $\mathbf{u}^{*}$, da equação da quantidade de movimento do sistema (3.6), sem considerar a condição de incompressibilidade:

$$
\begin{gathered}
\rho\left(\frac{\mathbf{u}^{*}-\mathbf{u}}{\Delta t}+(\mathbf{u} \cdot \mathbf{u})^{n}\right)=-\nabla q+\nabla \cdot[2 \mu \mathbf{d}]^{n}+\mathbf{f}^{n}, \\
\mathbf{C C}\left(\mathbf{u}^{*}\right)=\mathbf{h}_{\mathbf{u}^{*}},
\end{gathered}
$$

onde $q$ é uma aproximação para $p^{n+1}$ e $\mathbf{C C}$ é algum tipo de condição de contorno para a velocidade auxiliar.

\section{Passo 2:}

Projeção da velocidade auxiliar:

$$
\begin{gathered}
\mathbf{u}^{*}=\mathbf{u}^{n+1}+\frac{\Delta t}{\rho} \nabla \phi, \\
\nabla \cdot \mathbf{u}^{n+1}=0 .
\end{gathered}
$$


Para calcular $\mathbf{u}^{n+1}$ de (3.9) é necessário conhecer $\phi$. Aplicando-se o operador divergente à equação (3.9) e usando a igualdade (3.10), tem-se uma equação de Poisson para $\phi$

$$
\nabla^{2} \phi=\frac{\rho}{\Delta t} \nabla \cdot \mathbf{u}^{*}
$$

A solução desta equação será obtida uma vez definida a condição de contorno para $\phi$.

\section{Passo 3:}

Determinação da pressão (atualização da pressão). Para isto será usado o seguinte fato: equações (3.7) e (3.9) aproximam a equação (3.6). Portanto, substituindo-se (3.9) em (3.7) e igualando-se com a equação (3.6) obtém-se

$$
p^{n+1}=\phi+q .
$$

O método de projeção foi criado com o objetivo de tratar dificuldades que surgem do modo como as incógnitas $\mathbf{u}$ e $\rho$ estão acopladas em uma mesma equação. Tal acoplamento pode gerar sistemas muito grandes a serem resolvidos e que, em diversos casos, possuem matrizes malcondicionadas que interferem na convergência de métodos iterativos. Os métodos de projeção propõem a realização do cálculo de u e $\rho$ separadamente, o que facilita a resolução dos sistemas lineares necessários.

Desta forma, a equação de Poisson resultante do método de projeção tornou-se objeto desse estudo e a solução numérica dessas EDPs não-lineares, é apresentada no capítulo seguinte.

\subsection{Formulação de métodos}

Devido ao fato das equações diferenciais, que em geral, modelam problemas de fluxo de fluido que apresentam complexidade de se obter soluções analíticas, faz-se necessário a aplicação de técnicas numéricas para resolvê-las. Essas equações são substituídas por um sistema de equações algébricas. Na literatura, encontra-se diversas técnicas para obter o sistema de equações algébricas, como por exemplo, diferenças finitas [9], elementos finitos [41], volumes finitos [28] e métodos espectrais [27].

Nesse trabalho, escolheu-se a técnica de diferenças finitas compactas que será abordada a seguir.

\subsection{Esquema compacto de diferenças finitas}

Esta seção fornece a dedução de um esquema compacto de diferenças finitas aplicada à problemas elípticos, conforme o procedimento descrito em [24]. Será tratado problemas 1D e 2D com coeficientes variáveis de convecção. Para introduzir as idéias principais, considere a equação de convecção-difusão

$$
-a \frac{d^{2} \phi}{d x^{2}}+u \frac{d \phi}{d x}=S(x)
$$

com coeficientes a e $u$ constantes, termo fonte $S(x)$ e condição do contorno do tipo Dirichlet $\phi(0)=\phi_{0}$ e $\phi(1)=\phi_{1}$. Este problema modelo é representativo de uma classe de problemas de transporte. Suponha que o domínio [0;1] seja dividido em células uniformemente espaçadas de comprimento $h$ e que $\phi_{i}$ refere-se a aproximação de $\phi\left(x_{i}\right)$, onde $x_{i}$ é a 
coordenada do nó correspondente. Usando diferenças finitas centradas de segunda ordem, temos:

$$
-a \frac{\phi_{i-1}-2 \phi_{i}+\phi_{i+1}}{h^{2}}+u \frac{\phi_{i+1}-\phi_{i-1}}{2 h}-\tau_{i}=S\left(x_{i}\right)
$$

onde $\tau_{i}$ é o erro de truncamento em $i$ dado por

$$
\tau_{i}=\frac{h^{2}}{12}\left[2 u \frac{d^{3} \phi}{d x^{3}}-a \frac{d^{4} \phi}{d x^{4}}\right]+O\left(h^{4}\right)
$$

Obtemos um esquema de diferenças finitas centradas de ordem $O\left(h^{2}\right)$. Podemos observar em (3.15) que se o termo $\left[2 u \frac{d^{3} \phi}{d x^{3}}-a \frac{d^{4} \phi}{d x^{4}}\right]$ desaparecer, o esquema teria, pelo menos, ordem $O\left(h^{4}\right)$. Uma outra alternativa, segundo [24] e [42], seria o termo $\left[2 u \frac{d^{3} \phi}{d x^{3}}-a \frac{d^{4} \phi}{d x^{4}}\right]$ ser aproximado por derivadas de ordem $O\left(h^{4}\right)$. Então teríamos um esquema de quarta ordem. Para obter um esquema compacto de alta ordem, devemos aproximar as derivadas do lado direito de (3.15) e depois incluí-las na equação discreta. As relações para $\frac{d^{3} \phi}{d x^{3}}$ e $\frac{d^{4} \phi}{d x^{4}}$ podem ser obtidas derivando a equação (3.13)

$$
\begin{aligned}
\frac{d^{3} \phi}{d x^{3}} & =\frac{1}{a}\left(u \frac{d^{2} \phi}{d x^{2}}-\frac{d S}{d x}\right) \\
\frac{d^{4} \phi}{d x^{4}} & =\frac{1}{a}\left(u \frac{d^{3} \phi}{d x^{3}}-\frac{d^{2} S}{d x^{2}}\right) \\
& =\frac{u}{a^{2}}\left(u \frac{d^{2} \phi}{d x^{2}}-\frac{d S}{d x}\right)-\frac{1}{a} \frac{d^{2} S}{d x^{2}}
\end{aligned}
$$

Usando aproximações de diferenças finitas centradas de ordem $O\left(h^{2}\right)$ nas derivadas do lado direito de (3.16) a ordem de convergencência é aumentada para $O\left(h^{4}\right)$. Assim, o esquema resultante permanece compacto, como desejado. O termo fonte $S$ desempenha um papel importante nas aproximações $\frac{d^{3} \phi}{d x^{3}}$ e $\frac{d^{4} \phi}{d x^{4}}$. Se for possível calcular as derivadas de $S$ analíticamente, essa informação poderá ser usada nas equações (3.16), no entanto, se isso não for possível, podemos usar uma aproximação de diferenças finita centrada de ordem $O\left(h^{2}\right)$ para aproximar o valor de $\frac{d S}{d x}$ e $\frac{d S^{2}}{d x^{2}}$ que ainda resulta em um esquema de quarta ordem. Podemos repetir o procedimento acima apresentado para criar esquemas compactos de alguma ordem arbitrária.

\subsection{Equação elíptica 2D}

Abordaremos a partir de agora um problema de Poisson 2D com coeficientes variáveis

$$
a \frac{\partial^{2} \phi}{\partial x^{2}}+c \frac{\partial^{2} \phi}{\partial y^{2}}+d \frac{\partial \phi}{\partial x}+e \frac{\partial \phi}{\partial y}=f(x, y)
$$

sujeito à condições de contorno em $\partial \Omega$ de Dirichlet, onde $f(x, y)$ é o termo forçante, $\Omega$ é o domínio espacial, $a, c, d$ e $e$ são funções que serão definidas nos testes e $\phi$ é a incógnita. Como relatamos na seção (3.3), é de grande interesse resolver o problema (3.17), construiremos um esquema compacto de diferenças finitas para resolver problemas desse tipo.

\subsubsection{Formulação do esquema compacto de alta ordem}

Por conveniência na aplicação do método para resolver a equação (3.17), primeiro vamos considerar o caso em que $a, c, d$ e $e$ são constantes e o domínio discretizado em uma malha 
uniforme. Denotamos $\phi\left(x_{i}, y_{j}\right)$ por $\phi_{i, j}$ e, seguindo o procedimento adotado na seção (3.5), substituímos os operadores por aproximações de diferenças finitas centradas na equação (3.17), teremos

$$
a \delta_{x}^{2} \phi_{i, j}+c \delta_{y}^{2} \phi_{i, j}+d \delta_{x} \phi_{i, j}+e \delta_{y} \phi_{i, j}-\tau_{i, j}=f_{i, j}
$$

onde o erro de truncamento é igual a

$$
\tau_{i, j}=\frac{h^{2}}{12}\left[2\left(d \frac{\partial^{3} \phi}{\partial x^{3}}+e \frac{\partial^{3} \phi}{\partial y^{3}}\right)+\left(a \frac{\partial^{4} \phi}{\partial x^{4}}+c \frac{\partial^{4} \phi}{\partial y^{4}}\right)\right]_{i, j}+O\left(h^{4}\right) .
$$

Tabela 3.1: Definições de operadores de derivadas mistas em uma malha uniforme

\begin{tabular}{|c|c|}
\hline Operador & Fórmula \\
\hline \hline$\delta_{x} \delta_{y} \phi_{i, j}$ & $\frac{\phi_{i+1, j+1}-\phi_{i-1, j+1}-\phi_{i+1, j-1}+\phi_{i-1, j-1}}{4 h^{2}}$ \\
$\delta_{x}^{+} \delta_{y}^{+} \phi_{i, j}$ & $\frac{\phi_{i+1, j+1}-\phi_{i, j+1}-\phi_{i+1, j}-\phi_{i, j}}{h^{2}}$ \\
$\delta_{x}^{+} \delta_{y}^{-} \phi_{i, j}$ & $\frac{\phi_{i+1, j}-\phi_{i, j}-\phi_{i, j-1}+\phi_{i+1, j-1}}{h^{2}}$ \\
$\delta_{x}^{-} \delta_{y}^{+} \phi_{i, j}$ & $\frac{\phi_{i, j+1}-\phi_{i-1, j+1}-\phi_{i, j}+\phi_{i-1, j}}{h^{2}}$ \\
$\delta_{x}^{-} \delta_{y}^{-} \phi_{i, j}$ & $\frac{\phi_{i, j}-\phi_{i-1, j}-\phi_{i, j-1}+\phi_{i-1, j-1}}{h^{2}}$ \\
\hline$\delta_{x}^{2} \delta_{y} \phi_{i, j}$ & $\frac{2\left(\phi_{i, j+1}-\phi_{i, j-1}\right)+\phi_{i+1, j+1}-\phi_{i+1, j-1}+\phi_{i-1, j+1}-\phi_{i-1, j-1}}{2 h^{3}}$ \\
$\delta_{x}^{2} \delta_{y}^{+} \phi_{i, j}$ & $\frac{2\left(\phi_{i, j+1}-\phi_{i, j}\right)+\phi_{i+1, j+1}-\phi_{i+1, j}+\phi_{i-1, j+1}-\phi_{i-1, j}}{h^{3}}$ \\
$\delta_{x}^{2} \delta_{y}^{-} \phi_{i, j}$ & $\frac{2\left(\phi_{i, j}-\phi_{i, j-1}\right)+\phi_{i+1, j}-\phi_{i+1, j}+\phi_{i-1, j}-\phi_{i-1, j-1}}{h^{3}}$ \\
$\delta_{x} \delta_{y}^{2} \phi_{i, j}$ & $\frac{2\left(\phi_{i+1, j}-\phi_{i-1, j}\right)+\phi_{i+1, j+1}-\phi_{i-1, j+1}+\phi_{i+1, j-1}-\phi_{i-1, j-1}}{h^{3}}$ \\
$\delta_{x}^{+} \delta_{y}^{2} \phi_{i, j}$ & $\frac{2\left(\phi_{i+1, j}-\phi_{i-1, j}\right)+\phi_{i+1, j+1}-\phi_{i, j+1}+\phi_{i+1, j-1}-\phi_{i, j-1}}{h^{3}}$ \\
$\delta_{x}^{-} \delta_{y}^{2} \phi_{i, j}$ & $\frac{2\left(\phi_{i, j}-\phi_{i-1, j}\right)+\phi_{i, j+1}-\phi_{i-1, j+1}+\phi_{i, j-1}-\phi_{i-1, j-1}}{h^{3}}$ \\
\hline$\delta_{x}^{2} \delta_{y}^{2} \phi_{i, j}$ & $\frac{4 \phi_{i, j}-2\left(\phi_{i+1, j}+\phi_{i-1, j}+\phi_{i, j+1}+\phi_{i, j-1}\right)}{h^{4}}+$ \\
$\frac{\phi_{i+1, j+1}+\phi_{i+1, j-1}+\phi_{i-1, j+1}+\phi_{i-1, j-1}}{h^{4}}$
\end{tabular}

Como feito anteriormente, iremos agora calcular as aproximações das derivadas em (3.19) e incluir na equação discretizada (3.18). Derivando a equação (3.17), temos

$$
\begin{gathered}
\frac{\partial^{3} \phi}{\partial x^{3}}=\frac{1}{a}\left(\frac{\partial f}{\partial x}-c \frac{\partial^{3} \phi}{\partial x \partial y^{2}}-d \frac{\partial^{2} \phi}{\partial x^{2}}-e \frac{\partial^{2}}{\partial x \partial y}\right) \\
\frac{\partial^{4} \phi}{\partial x^{4}}=\frac{1}{a}\left(\frac{\partial^{2} f}{\partial x^{2}}-c \frac{\partial^{4} \phi}{\partial x^{2} \partial y^{2}}-d \frac{\partial^{3} \phi}{\partial x^{3}}-e \frac{\partial^{3}}{\partial x^{2} \partial y}\right) \\
\frac{\partial^{3} \phi}{\partial y^{3}}=\frac{1}{c}\left(\frac{\partial f}{\partial y}-a \frac{\partial^{3} \phi}{\partial x^{2} \partial y}-d \frac{\partial^{2} \phi}{\partial x \partial y}-e \frac{\partial^{2}}{\partial y^{2}}\right) \\
\frac{\partial^{4} \phi}{\partial y^{4}}=\frac{1}{c}\left(\frac{\partial^{2} f}{\partial y^{2}}-a \frac{\partial^{4} \phi}{\partial x^{2} \partial y^{2}}-d \frac{\partial^{3} \phi}{\partial x \partial y^{2}}-e \frac{\partial^{3}}{\partial y^{3}}\right)
\end{gathered}
$$


Note que todos os termos do lado direito da equação (3.20) tem ordem $O\left(h^{2}\right)$ de aproximação (ver tabela 3.1 a lista completa dos operadores de derivadas mistas). Por exemplo

$$
\left.\frac{\partial^{3} \phi}{\partial x \partial y^{2}}\right|_{i, j}=\delta_{x} \delta_{y}^{2} \phi_{i, j}-\frac{h^{2}}{12}\left[2 \frac{\partial^{5} \phi}{\partial x \partial y^{4}}\right]_{i, j}+O\left(h^{4}\right),
$$

Assim, $\frac{\partial^{3} \phi}{\partial x^{3}}$ pode ser aproximado com ordem $O\left(h^{2}\right)$. Analogamente todos os termos do lado direito da equação (3.21) tem ordem $O\left(h^{2}\right)$ de aproximação, então $\frac{\partial^{4} \phi}{\partial x^{4}}$ pode ser aproximado com ordem $O\left(h^{2}\right)$. O mesmo acontece com $\frac{\partial^{3} \phi}{\partial y^{3}}$ e $\frac{\partial^{4} \phi}{\partial y^{4}}$. Diante disso temos que as equações (3.20)-(3.23) têm ordem $O\left(h^{2}\right)$ de aproximação.

Substituindo as equações (3.20)-(3.23) na equação (3.19) e simplificando, temos que o erro de truncamento fica

$$
\begin{aligned}
\tau_{i j}= & \frac{h^{2}}{12}\left[-\frac{2 d^{2}}{a} \frac{\partial^{2} \phi}{\partial x^{2}}-\frac{2 d e c+a e d}{a c} \frac{\partial^{2} \phi}{\partial x \partial y}-\frac{e^{2}}{c} \frac{\partial^{2} \phi}{\partial y^{2}}-(a+c) \frac{\partial^{4} \phi}{\partial x^{2} \partial y^{2}}-\frac{2 d c+a d}{a} \frac{\partial^{3} \phi}{\partial x \partial y^{2}}\right]+ \\
& \frac{h^{2}}{12}\left[-\frac{e a+e c}{c} \frac{\partial^{3} \phi}{\partial x^{2} \partial y}-d \frac{\partial^{3} \phi}{\partial x^{3}}-e \frac{\partial^{3} \phi}{\partial y^{3}}+\frac{\partial^{2} f}{\partial x^{2}}+\frac{\partial^{2} f}{\partial y^{2}}+\frac{2 d}{a} \frac{\partial f}{\partial x}+\frac{e}{c} \frac{\partial f}{\partial y}\right]
\end{aligned}
$$

Agora, substituindo as derivadas em (3.25) por aproximações de ordem $O\left(h^{2}\right)$ e inserindoas na equação (3.18), temos

$$
\begin{gathered}
-\left(1+\frac{a^{2} h^{2}}{12}\right) \delta_{x}^{2} \phi_{i j}-\left(1+\frac{c^{2} h^{2}}{12}\right) \delta_{y}^{2} \phi_{i j}+a \delta_{x} \phi_{i j}+c \delta_{y} \phi_{i j}- \\
\frac{h^{2}}{6}\left[\delta_{x}^{2} \delta_{y}^{2} \phi_{i j}-a \delta_{x} \delta_{y}^{2} \phi_{i j}-c \delta_{x}^{2} \delta_{y} \phi_{i j}+a c \delta_{x} \delta_{y} \phi_{i j}\right]= \\
f_{i j}+\frac{h^{2}}{12}\left[\frac{\partial^{2} f}{\partial x^{2}}+\frac{\partial^{2} f}{\partial y^{2}}-a \frac{\partial f}{\partial x}-c \frac{\partial f}{\partial y}\right]_{i j}+O\left(h^{4}\right) .
\end{gathered}
$$

Se os coeficientes $a, c, d$ e $e$ são variáveis ao longo do domínio, o procedimento para se chegar no esquema compacto é o mesmo, porém exige um pouco mais de manipulação algébrica para derivar as fórmulas resultantes do erro de truncamento [42].

No caso onde os coeficientes $a, c, d$ e $e$ são variáveis, temos o seguinte esquema compacto de $O\left(h^{4}\right)$

$$
\begin{aligned}
& A_{i j}^{*} \delta_{x}^{2} \phi_{i j}+B_{i j}^{*} \delta_{x} \delta_{y} \phi_{i j}+C_{i j}^{*} \delta_{y}^{2} \phi_{i j}+D_{i j}^{*} \delta_{x} \phi_{i j}+E_{i j}^{*} \delta_{y} \phi_{i j}+F_{i j}^{*} \phi_{i j}+P_{i j}^{*} \delta_{x}^{2} \delta_{y}^{2} \phi_{i j}+Q_{i j}^{*} \delta_{x}^{2} \delta_{y} \phi_{i j} \\
& +R_{i j}^{*} \delta_{x} \delta_{y}^{2} \phi_{i j}=G_{i j}^{*}
\end{aligned}
$$

onde os valores para $A_{i j}^{*}, B_{i j}^{*}, C_{i j}^{*}, D_{i j}^{*}, E_{i j}^{*}, F_{i j}^{*}, P_{i j}^{*}, Q_{i j}^{*}, R_{i j}^{*}$ e $G_{i j}^{*}$ pode ser encontrado seguindo o procedimento a seguir.

Primeiro, temos a seguinte equação

$$
a(x, y) \frac{\partial^{2} \phi}{\partial x^{2}}+c(x, y) \frac{\partial^{2} \phi}{\partial y^{2}}+d(x, y) \frac{\partial \phi}{\partial x}+e(x, y) \frac{\partial \phi}{\partial y}+f(x, y) \phi=g(x, y)
$$

sujieto à condições de contorno em $\partial \Omega$ de Dirichlet, onde $g(x, y)$ é o termo forçante, $\Omega$ é o domínio espacial, $a, c, d, e$ e $f$ são funções que serão definidas nos testes e $\phi$ é a incógnita.

Substituindo os operadores por aproximações de diferenças finitas centradas na equação 3.28 temos o esquema compacto de diferenças finitas de segunda ordem

$$
a_{i j} \delta_{x}^{2} \phi_{i j}+c_{i j} \delta_{y}^{2} \phi_{i j}+d_{i j} \delta_{x} \phi_{i j}+e_{i j} \delta_{y} \phi_{i j}+f_{i j} \phi_{i j}+\tau_{i j}=g_{i j}
$$


com o erro local de truncamento dado por

$$
\tau_{i j}=-\frac{1}{12}\left[a_{i j} h_{x}^{2}{\frac{\partial^{4} \phi}{\partial x^{4}}}_{i j}+c_{i j} h_{y}^{2}{\frac{\partial^{4} \phi}{\partial y^{4}}}_{i j}+2 d_{i j} h_{x}^{2}{\frac{\partial^{3} \phi}{\partial x^{3}}}_{i j}+2 e_{i j} h_{y}^{2}{\frac{\partial^{3} \phi}{\partial y^{3}}}_{i j}\right]+O\left(h^{4}\right) .
$$

Para obter um esquema compacto de quarta ordem seguimos a seguinte metodologia:

Derivando a equação 3.28 em relação a $x$, temos

$\frac{\partial^{3} \phi}{\partial x^{3}}=\frac{1}{a}\left[\frac{\partial g}{\partial x}-\left(\frac{\partial a}{\partial x}+d\right) \frac{\partial^{2} \phi}{\partial x^{2}}-e \frac{\partial^{2} \phi}{\partial x \partial y}-c \frac{\partial^{3} \phi}{\partial x \partial y^{3}}-\frac{\partial c}{\partial x} \frac{\partial^{2} \phi}{\partial y^{2}}-\left(\frac{\partial d}{\partial x}+f\right) \frac{\partial \phi}{\partial x}-\frac{\partial e}{\partial x} \frac{\partial \phi}{\partial y}-\frac{\partial f}{\partial x} \phi\right]$.

analogamente, derivando a equação 3.28 em relação a $y$, temos

$\frac{\partial^{3} \phi}{\partial y^{3}}=\frac{1}{c}\left[\frac{\partial g}{\partial y}-\left(\frac{\partial c}{\partial y}+e\right) \frac{\partial^{2} \phi}{\partial y^{2}}-a \frac{\partial^{3} \phi}{\partial x^{2} \partial y}-\frac{\partial a}{\partial y} \frac{\partial^{2} \phi}{\partial x^{2}}-d \frac{\partial^{2} \phi}{\partial x \partial y}-\frac{\partial d}{\partial y} \frac{\partial \phi}{\partial x}-\left(\frac{\partial e}{\partial y}+f\right) \frac{\partial \phi}{\partial y}-\frac{\partial f}{\partial y} \phi\right]$.

depois, derivando a equação 3.31 em relação a $x$, temos

$$
\begin{aligned}
\frac{\partial^{4} \phi}{\partial x^{4}}= & \frac{1}{a}\left[\frac{\partial^{2} g}{\partial x^{2}}-\left(2 \frac{\partial a}{\partial x}+d\right) \frac{\partial^{3} \phi}{\partial x^{3}}-\left(\frac{\partial^{2} a}{\partial x^{2}}+2 \frac{\partial d}{\partial x}+f\right) \frac{\partial^{2} \phi}{\partial x^{2}}-e \frac{\partial^{3} \phi}{\partial x^{2} \partial y}-2 \frac{\partial e}{\partial x} \frac{\partial^{2} \phi}{\partial x \partial y}+\right. \\
& \left.-c \frac{\partial^{4} \phi}{\partial x^{2} \partial y^{2}}-2 \frac{\partial c}{\partial x} \frac{\partial^{3} \phi}{\partial x \partial y^{2}}-\frac{\partial^{2} c}{\partial x^{2}} \frac{\partial^{2} \phi}{\partial y^{2}}-\left(\frac{\partial^{2} d}{\partial x^{2}}+2 \frac{\partial f}{\partial x}\right) \frac{\partial \phi}{\partial x}-\frac{\partial e}{\partial x^{2}} \frac{\partial \phi}{\partial y}-\frac{\partial^{2} f}{\partial x^{2}} \phi\right] .
\end{aligned}
$$

seguindo o raciocínio anterior, derivamos a equação 3.32 em relação a y

$$
\begin{aligned}
\frac{\partial^{4} \phi}{\partial y^{4}}= & \frac{1}{c}\left[\frac{\partial^{2} g}{\partial y^{2}}-\left(2 \frac{\partial c}{\partial y}+e\right) \frac{\partial^{3} \phi}{\partial y^{3}}-\left(\frac{\partial^{2} c}{\partial y^{2}}+2 \frac{\partial e}{\partial y}+f\right) \frac{\partial^{2} \phi}{\partial y^{2}}-d \frac{\partial^{3} \phi}{\partial x \partial y^{2}}-2 \frac{\partial a}{\partial y} \frac{\partial^{3} \phi}{\partial x^{2} \partial y}+\right. \\
& \left.-\frac{\partial^{2} a}{\partial y^{2}} \frac{\partial^{2} \phi}{\partial x^{2}}-a \frac{\partial^{4} \phi}{\partial x^{2} \partial y^{2}}-2 \frac{\partial d}{\partial y} \frac{\partial^{2} \phi}{\partial x \partial y}-\frac{\partial^{2} d}{\partial y^{2}} \frac{\partial \phi}{\partial x}-\left(\frac{\partial^{2} e}{\partial y^{2}}+2 \frac{\partial f}{\partial y}\right) \frac{\partial \phi}{\partial y}-\frac{\partial^{2} f}{\partial y^{2}} \phi\right] .
\end{aligned}
$$

Usando diferenças finitas centradas em $\left(x_{i}, y_{j}\right)$ para $u_{x x x}, u_{y y y}, u_{x x x x}$ e $u_{y y y y}$, temos

$$
\begin{aligned}
\frac{\partial^{3} \phi}{\partial x^{3} i j}= & \frac{1}{a_{i j}}\left[\delta_{x} g_{i j}-\left(\delta_{x} a_{i j}+d_{i j}\right) \delta_{x}^{2} \phi_{i j}-e_{i j} \delta_{x} \delta_{y} \phi_{i j}-c_{i j} \delta_{x} \delta_{y}^{2} \phi_{i j}-\delta_{x} c_{i j} \delta_{y}^{2} \phi_{i j}+\right. \\
& \left.-\left(\delta_{x} d_{i j}+f_{i j}\right) \delta_{x} \phi_{i j}-\delta_{x} e_{i j} \delta_{y} \phi_{i j}-\delta_{x} f_{i j} \phi_{i j}\right]+O\left(h_{x}^{2}+h_{x} h_{y}+h_{y}^{2}\right) . \\
\frac{\partial^{3} \phi}{\partial y^{3} i j}= & \frac{1}{c_{i j}}\left[\delta_{y} g_{i j}-\left(\delta_{y} c_{i j}+e_{i j}\right) \delta_{y}^{2} \phi_{i j}-a_{i j} \delta_{x}^{2} \delta_{y} \phi_{i j}-\delta_{y} a_{i j} \delta_{x}^{2} \phi_{i j}-d_{i j} \delta_{x} \delta_{y} \phi_{i j}+\right. \\
& \left.-\delta_{y} d_{i j} \delta_{x} \phi_{i j}-\left(\delta_{y} e_{i j}+f_{i j}\right) \delta_{y} \phi_{i j}-\delta_{y} f_{i j} \phi_{i j}\right]+O\left(h_{x}^{2}+h_{x} h_{y}+h_{y}^{2}\right) . \\
\frac{\partial^{4} \phi}{\partial x^{4} i j}= & \frac{1}{a_{i j}}\left[\delta_{x}^{2} g_{i j}-\left(2 \delta_{x} a_{i j}+d_{i j}\right)\left(\frac{\partial^{3} \phi}{\partial x^{3}}\right)_{i j}-\left(\delta_{x}^{2} a_{i j}+2 \delta_{x} d_{i j}+f_{i j}\right) \delta_{x}^{2} \phi_{i j}+\right. \\
& -e_{i j} \delta_{x}^{2} \delta_{y} \phi_{i j}-2 \delta_{x} e_{i j} \delta_{x} \delta_{y} \phi_{i j}-c_{i j} \delta_{x}^{2} \delta_{y}^{2} \phi_{i j}-2 \delta_{x} c_{i j} \delta_{x} \delta_{y}^{2} u_{i j}-\delta_{x}^{2} c_{i j} \delta_{y}^{2} \phi_{i j}+ \\
& \left.-\left(\delta_{x}^{2} d_{i j}+2 \delta_{x} f_{i j}\right) \delta_{x} \phi_{i j}-\delta_{x}^{2} e_{i j} \delta_{y} \phi_{i j}-\delta_{x}^{2} f_{i j} \phi_{i j}\right]+O\left(h_{x}^{2}+h_{x} h_{y}+h_{y}^{2}\right) . \\
\frac{\partial^{4} \phi}{\partial y^{4}}{ }_{i j}= & \frac{1}{c_{i j}}\left[\delta_{y}^{2} g_{i j}-\left(2 \delta_{y} c_{i j}+e_{i j}\right)\left(\frac{\partial^{3} \phi}{\partial y^{3}}\right)_{i j}-\left(\delta_{y}^{2} c_{i j}+2 \delta_{y} e_{i j}+f_{i j}\right) \delta_{y}^{2} \phi_{i j}+\right. \\
& -d_{i j} \delta_{x} \delta_{y}^{2} \phi_{i j}-2 \delta_{y} a_{i j} \delta_{x}^{2} \delta_{y} \phi_{i j}-\delta_{y}^{2} a_{i j} \delta_{x}^{2} \phi_{i j}-a_{i j} \delta_{x}^{2} \delta_{y}^{2} \phi_{i j}-2 \delta_{y} d_{i j} \delta_{x} \delta_{y} \phi_{i j}+ \\
& \left.-\delta_{y}^{2} d_{i j} \delta_{x} \phi_{i j}-\left(\delta_{y}^{2} e_{i j}+2 \delta_{y} f_{i j}\right) \delta_{y} \phi_{i j}-\delta_{y}^{2} f_{i j} \phi_{i j}\right]+O\left(h_{x}^{2}+h_{x} h_{y}+h_{y}^{2}\right) .
\end{aligned}
$$


Finalmente, substituindo as equações 3.35 - 3.38 na equação 3.30 , combinando com a equação 3.29 , temos

$$
\begin{aligned}
& A_{i j}^{*} \delta_{x}^{2} \phi_{i j}+B_{i j}^{*} \delta_{x} \delta_{y} \phi_{i j}+C_{i j}^{*} \delta_{y}^{2} \phi_{i j}+D_{i j}^{*} \delta_{x} \phi_{i j}+E_{i j}^{*} \delta_{y} \phi_{i j}+F_{i j}^{*} \phi_{i j}+P_{i j}^{*} \delta_{x}^{2} \delta_{y}^{2} \phi_{i j}+Q_{i j}^{*} \delta_{x}^{2} \delta_{y} \phi_{i j} \\
& +R_{i j}^{*} \delta_{x} \delta_{y}^{2} \phi_{i j}=G_{i j}^{*}
\end{aligned}
$$

onde:

$$
\begin{aligned}
& A_{i j}^{*}=a_{i j}+\frac{1}{12}\left[h_{x}^{2}\left(\delta_{x}^{2} a_{i j}+2 \delta_{x} d_{i j}+f_{i j}+\alpha_{i j}\left(\delta_{x} a_{i j}+d_{i j}\right)\right)+h_{y}^{2}\left(\delta_{y}^{2} a_{i j}+\beta_{i j} \delta_{y} a_{i j}\right)\right] \\
& B_{i j}^{*}=\frac{1}{12}\left[h_{x}^{2}\left(\alpha_{i j} e_{i j}+2 \delta_{x} e_{i j}\right)+h_{y}^{2}\left(\beta_{i j} d_{i j}+2 \delta_{y} d_{i j}\right)\right] \\
& C_{i j}^{*}=c_{i j}+\frac{1}{12}\left[h_{y}^{2}\left(\delta_{y}^{2} c_{i j}+2 \delta_{y} e_{i j}+f_{i j}+\beta_{i j}\left(\delta_{y} c_{i j}+e_{i j}\right)\right)+h_{x}^{2}\left(\delta_{x}^{2} c_{i j}+\alpha_{i j} \delta_{x} c_{i j}\right)\right] \\
& D_{i j}^{*}=d_{i j}+\frac{1}{12}\left[h_{x}^{2}\left(\delta_{x}^{2} d_{i j}+2 \delta_{x} f_{i j}+\alpha_{i j}\left(\delta_{x} d_{i j}+f_{i j}\right)\right)+h_{y}^{2}\left(\delta_{y}^{2} d_{i j}+\beta_{i j} \delta_{y} d_{i j}\right)\right] \\
& E_{i j}^{*}=e_{i j}+\frac{1}{12}\left[h_{y}^{2}\left(\delta_{y}^{2} e_{i j}+2 \delta_{y} f_{i j}+\beta_{i j}\left(\delta_{y} e_{i j}+f_{i j}\right)\right)+h_{x}^{2}\left(\delta_{x}^{2} e_{i j}+\alpha_{i j} \delta_{x} e_{i j}\right)\right] \\
& F_{i j}^{*}=f_{i j}+\frac{1}{12}\left[h_{x}^{2}\left(\delta_{x}^{2} f_{i j}+\alpha_{i j} \delta_{x} f_{i j}\right)+h_{y}^{2}\left(\delta_{y}^{2} f_{i j}+\beta_{i j} \delta_{y} f_{i j}\right)\right] \\
& P_{i j}^{*}=\frac{1}{12}\left(h_{y}^{2} a_{i j}+h_{x}^{2} c_{i j}\right) \\
& Q_{i j}^{*}=\frac{1}{12}\left[h_{x}^{2} e_{i j}+h_{y}^{2}\left(2 \delta_{y} a_{i j}+\beta_{i j} a_{i j}\right)\right] \\
& R_{i j}^{*}=\frac{1}{12}\left[h_{y}^{2} d_{i j}+h_{x}^{2}\left(2 \delta_{x} c_{i j}+\alpha_{i j} c_{i j}\right)\right] \\
& G_{i j}^{*}=g_{i j}+\frac{1}{12}\left[h_{x}^{2}\left(\delta_{x}^{2} g_{i j}+\alpha_{i j} \delta_{x} g_{i j}\right)+h_{y}^{2}\left(\delta_{y}^{2} g_{i j}+\beta_{i j} \delta_{y} g_{i j}\right)\right] \\
& \alpha_{i j}=\frac{d_{i j}-2 \delta_{x} a_{i j}}{a_{i j}} \quad \beta_{i j}=\frac{e_{i j}-2 \delta_{y} c_{i j}}{c_{i j}}
\end{aligned}
$$

Assim, a equação 3.39 com as equações 3.40 - 3.50 formam o esquema compacto de quarta ordem para resolver a equação elíptica com coeficientes variávies 3.28. O esquema pode ser escrito como um método de nove pontos, vejamos

$l_{0} u_{i j}+l_{1} u_{i+1, j}+l_{2} u_{i, j+1}+l_{3} u_{i-1, j}+l_{4} u_{i, j-1}+l_{5} u_{i+1, j+1}+l_{6} u_{i-1, j+1}+l_{7} u_{i-1, j-1}+l_{8} u_{i+1, j-1}=G_{i j}^{*}$

onde

$$
\begin{gathered}
l_{0}=-\frac{2}{h_{x}^{2}} A_{i j}^{*}-\frac{2}{h_{y}^{2}} C_{i j}^{*}+F_{i j}^{*}+\frac{4}{h_{x}^{2} h_{y}^{2}} P_{i j}^{*} \\
l_{1}=\frac{1}{h_{x}^{2}} A_{i j}^{*}+\frac{1}{2 h_{x}} D_{i j}^{*}-\frac{2}{h_{x}^{2} h_{y}^{2}} P_{i j}^{*}-\frac{1}{h_{x} h_{y}^{2}} R_{i j}^{*} \\
l_{2}=\frac{1}{h_{y}^{2}} C_{i j}^{*}+\frac{1}{2 h_{y}} E_{i j}^{*}-\frac{2}{h_{x}^{2} h_{y}^{2}} P_{i j}^{*}-\frac{1}{h_{x}^{2} h_{y}} Q_{i j}^{*}
\end{gathered}
$$




$$
\begin{gathered}
l_{3}=\frac{1}{h_{x}^{2}} A_{i j}^{*}-\frac{1}{2 h_{x}} D_{i j}^{*}-\frac{2}{h_{x}^{2} h_{y}^{2}} P_{i j}+\frac{1}{h_{x} h_{y}^{2}} R_{i j}^{*} \\
l_{4}=\frac{1}{h_{y}^{2}} C_{i j}^{*}-\frac{1}{2 h_{y}} E_{i j}^{*}-\frac{2}{h_{x}^{2} h_{y}^{2}} P_{i j}^{*}+\frac{1}{h_{x}^{2} h_{y}} Q_{i j}^{*} \\
l_{5}=\frac{1}{4 h_{x} h_{y}} B_{i j}^{*}+\frac{1}{h_{x}^{2} h_{y}^{2}} P_{i j}^{*}+\frac{1}{2 h_{x}^{2} h_{y}} Q_{i j}^{*}+\frac{1}{2 h_{x} h_{y}^{2}} R_{i j}^{*} \\
l_{6}=-\frac{1}{4 h_{x} h_{y}} B_{i j}^{*}+\frac{1}{h_{x}^{2} h_{y}^{2}} P_{i j}^{*}+\frac{1}{2 h_{x}^{2} h_{y}} Q_{i j}^{*}-\frac{1}{2 h_{x} h_{y}^{2}} R_{i j}^{*} \\
l_{7}=\frac{1}{4 h_{x} h_{y}} B_{i j}^{*}+\frac{1}{h_{x}^{2} h_{y}^{2}} P_{i j}^{*}-\frac{1}{2 h_{x}^{2} h_{y}} Q_{i j}^{*}-\frac{1}{2 h_{x} h_{y}^{2}} R_{i j}^{*} \\
l_{8}=-\frac{1}{4 h_{x} h_{y}} B_{i j}^{*}+\frac{1}{h_{x}^{2} h_{y}^{2}} P_{i j}^{*}-\frac{1}{2 h_{x}^{2} h_{y}} Q_{i j}^{*}+\frac{1}{2 h_{x} h_{y}^{2}} R_{i j}^{*}
\end{gathered}
$$

com o erro local de truncamento: $O\left(h_{x}^{4}+h_{x}^{3} h_{y}+h_{x}^{2} h_{y}^{2}+h_{x} h_{y}^{3}+h_{y}^{4}\right)$.

Podemos observar que para os dois esquemas compactos apresentados para o caso $2 D$ envolvem um stencil de nove pontos, isso devido necessidade de introduzir a derivada mista de $\phi$ na formulação do método. No entanto, segundo [42] devido o método ser compacto, há um pequeno aumento na largura da banda da matriz resultante. Por exemplo, para um domínio quadrado de $N$ pontos na malha, Spotz [42] afirma que para o método de diferenças finitas clássico de $O\left(h^{4}\right)$ a meia largura de banda é dado por $\sqrt{N}+1$ enquanto que para o esquema compacto de ordem $O\left(h^{4}\right)$ a meia largura de banda é $\sqrt{N}+2$.

Spotz [42] também evidencia que, para o esquema compacto, os coeficientes de matriz o vetor do lado direito são mais complicados de se obter, pois há um número de operações adicionais comparado com o método clássico. O número de multiplicações e divisões necessárias para calcular o sistema, do esquema compacto para o caso com coeficientes variáveis, é de aproximadamente $56 \mathrm{~N}$, em comparação com aproximadamente $6 \mathrm{~N}$ para o método clássico. Embora este seja um aumento de mais de nove vezes nas operações, esta comparação é insignificante e não deve ser levada em consideração ao se escolher um método, pois o ganho que se tem com o esquema compacto é mais valioso quando estamos comparando o aumento na precisão de aproximação da solução numérica, o que nos permite trabalhar com malhas mais grossas. 


\section{Capítulo 4}

\section{Resultados e discussões}

Este Capítulo apresenta os resultados obtidos com o código implementado ao longo deste trabalho. São mostradas resoluções numéricas de problemas elípticos com coeficientes constantes e variáveis e, em ambos casos, foram consideradas discretizações do domínio em malhas uniformes e malhas hierárquicas (malhas compostas). A convergência e precisão do método foram verificadas usando a estratégia de solução manufaturada.

\subsection{Normas e análise de convergência numérica em ma- lhas regulares e em malhas compostas}

Antes de apresentarmos qualquer resultado numérico, é necessário descrevermos as normas com as quais avaliaremos os erros tanto em malhas uniformes quanto em malhas compostas. Além disso, descreveremos como será efetuada a análise da convergência numérica. Primeiro, definimos as normas $\|\cdot\|_{1},\|\cdot\|_{2}$ e $\|\cdot\|_{\infty}$ de uma variável escalar $\phi$ definida em $\Omega$ como

$$
\begin{gathered}
\|\phi\|_{q}=\left(\sum_{k \in \Omega}\left|\phi_{k}\right|^{q} h_{k}^{2}\right)^{1 / q}, \quad q=1,2, \\
\|\phi\|_{\infty}=\max _{k \in \Omega}\left|\phi_{k}\right|
\end{gathered}
$$

onde $\phi_{k}$ é o valor assumido por $\phi$ no centro da k-ésima célula cujas arestas medem $\delta_{x}=$ $\delta_{y}=h_{k}$ (a igualdade é apenas para simplificar a apresentação e não representa nenhuma limitação das definições). Se definirmos $\psi=\left(\psi_{1}, \psi_{2}\right)$, uma variável vetorial, então $\psi_{1}$ estará posicionada em $\Omega_{u}$ e $\psi_{2}$ em $\Omega_{v}$ e

$$
\begin{gathered}
\|\psi\|_{q}=\left(\sum_{i \in \Omega_{u}}\left|\psi_{1, i}\right|^{q} a_{i}+\sum_{j \in \Omega_{v}}\left|\psi_{2, j}\right|^{q} b_{j}\right)^{1 / q}, \quad q=1,2, \\
\|\psi\|_{\infty}=\max \left\{\max _{i \in \Omega_{u}}\left\{\left|\psi_{1, i}\right|\right\}, \max _{j \in \Omega_{v}}\left\{\left|\psi_{2, j}\right|\right\}\right\}
\end{gathered}
$$

onde

$$
a_{i}=\left\{\begin{array}{lll}
H^{2} & , & \text { nas arestas grossas } \\
H^{2} / r^{2} & , & \text { nas arestas finas } \\
H^{2}(r+1) /\left(2 r^{2}\right) & , & \text { nas arestas de interface fina-grossa }
\end{array}\right.
$$


sendo $r$ a razão de refinamento entre os níveis $l$ e $l+1$ e $H$, o espaçamento da malha no nível $l$. Definimos os pesos $b_{j}$ de maneira similar. Para efetuarmos a análise de convergência numérica, assumiremos que a solução numérica obtida pelo método possui uma expansão assintótica em potências do espaçamento da malha. Em uma malha uniforme, por exemplo, uma variável escalar $\phi$ tem solução numérica $\phi(x, t, h)$ a qual, por hipótese, pode ser escrita na forma

$$
\phi(x, t, h)=\phi_{\epsilon}(x, t)+E_{q}(x, t) h^{q}+E_{q+1}(x, t) h^{q+1}+\cdots
$$

para todos os espaçamentos $h$ suficientemente pequenos, onde $\phi_{\epsilon}(x, t)$ é a solução exata e as funções que definem os coeficientes $E_{i}, i=q, q+1, \ldots$, independem de $h$. Nos problemas apresentados na seção a seguir, a solução exata é conhecida. Para avaliarmos a ordem de convergência, depois de calcularmos um valor aproximado $\phi(x, t, h)$ para um dado espaçamento $h$, recalculamos para o mesmo ponto da malha $(x, t)$ uma nova aproximação, desta com espaçamento $h / 2$, a aproximação $\phi(x, t, h / 2)$. Assim, para espaçamentos suficientemente pequenos, em primeira aproximação, teremos

$$
\begin{gathered}
\phi(x, t, h) \approx \phi_{\epsilon}(x, t)+E_{q}(x, t) h^{q}, \\
\phi(x, t, h / 2) \approx \phi_{\epsilon}(x, t)+E_{q}(x, t) \frac{h^{q}}{2^{q}},
\end{gathered}
$$

o que nos fornece a aproximação para a razão de convergência

$$
\frac{\left\|\phi(x, t, h)-\phi_{\epsilon}(x, t)\right\|}{\left\|\phi(x, t, h / 2)-\phi_{\epsilon}(x, t)\right\|} \approx 2^{q},
$$

onde $\|\cdot\|$ representa a norma (4.1) ou (4.2). Observe que as razões de convergência 2, 4, 8, ..., significam que o método numérico tem ordem de precisão $q=1,2,3, \ldots$ Empregaremos a estimativa da razão de convergência (4.7) tanto em malhas uniformes quanto em malhas compostas. Quando necessário, faremos observações suplementares sobre o cálculo de normas e sobre a análise de convergência numérica empregando refinamento de malha.

\subsection{Representação matricial das discretizações}

A matriz que representa alguma discretização tem uma dimensão que depende da quantidade de células visíveis, pois é nelas que estão definidas as equações. Se o sistema é para uma variável então a dimensão da matriz é o número de células visíveis da variável. Se a matriz é para resolver sistema linear acoplado com duas variáveis, então a dimensão da matriz é a soma da quantidade de células visíves para cada variável.

A matriz que tem a discretização é definida seguindo a mesma estrutura de lista ligada para cada célula computacional. Quando a matriz já está definida na estrutura de dados, pode ser passada para uma biblioteca externa, como o PETSc, seguindo apropriadamente a maneira de se fazer a interface com cada biblioteca.

A matriz tem dimensão do número de células visíveis e devemos ter um mapa que relaciona cada posição das variáveis visíveis com seu índice de enumeração e com isto evitamos realizar buscas [40].

Para definir uma outra matriz de discretização, temos que seguir os mesmos passos: criar a estrutura matricial que armazena em cada célula uma lista ligada a um estêncil e depois, realizar em cada linha da matriz a chamada com a respectiva lista ligada de células e o peso da interpolação. 
Essa matriz é depois passada para o PETSc, que resolve o sistema segundo as opções que nós desejamos. Com pré-condicionador ou sem, com paralelismo ou não e com o solver selecionado.

\subsection{Problema elíptico com coeficientes constantes}

Para analisar o desempenho do Esquema Compacto de Quarta Ordem, consideramos a seguinte equação elíptica:

$$
\Delta \varphi(x, y)=f(x, y), \quad(x, y) \in[0,1] \times[0,1]
$$

sujeito à condição de contorno de Dirichlet.

$$
\left\{\begin{array}{lll}
\varphi(0, y)=0 & \text { para } & 0 \leq y \leq 1 \\
\varphi(1, y)=0 & \text { para } & 0 \leq y \leq 1 \\
\varphi(x, 0)=0 & \text { para } & 0 \leq x \leq 1 \\
\varphi(x, 1)=0 & \text { para } & 0 \leq x \leq 1
\end{array}\right.
$$

A solução exata para este problema é

$$
\begin{aligned}
& \varphi(x, y)=\sin (2 \pi x) \sin (2 \pi y) \\
& f(x, y)=-8 \pi^{2} \sin (2 \pi x) \sin (2 \pi y)
\end{aligned}
$$

Discretizamos o problema (4.8) em malha uniforme e composta tanto usando o Esquema Compacto de Quarta Ordem (ECQO) descrito na seção anterior quanto o Método de Diferenças Finitas padrão de quarta ordem (MDF) a fim de comparar a performance dos dois métodos.

\subsubsection{Teste para malha uniforme}

A ordem de convergência $(q)$ esperada para os métodos MDF e ECDF é quatro, o que foi comprovada numericamente. Os resultados obtidos encontram-se na Tabela (4.1). A solução do sistema linear dado pela discretização do problema foi computada com o uso do PETSc com o método GMRES e pré-condicionador PCJACOBI. Adotamos tolerância de $10^{-16}$, número máximo de iterações de $10^{4}$. A coluna Iter na tabela fornece o número de iterações que o Método GMRES, com pré-condicionador PCJACOBI do PETSc [40] precisou para obter os resultados apresentados. Na Tabela (4.1) considere

$$
r_{e}=\frac{\left\|u(x, y, h)-u_{e}(x, y)\right\|}{\left\|u(x, y, h / 2)-u_{e}(x, y)\right\|} .
$$

Fica evidente, pelos resultados apresentados na Tabela 4.1, que o ECDF para problemas bidimensionais é bem mais eficiente que o MDF, pois temos que obter a solução numérica de um sistema linear com bem menos incógnitas, para o mesmo erro de aproximação da solução do Problema de Poisson, levando a um menor esforço computacional. Além disso, o número de iterações para o esquema compacto é consideravelmente menor quando comparado com o método de diferenças finitas. 


\begin{tabular}{c|ccc|ccc}
\hline Malha & \multicolumn{3}{|c|}{ MDF } & \multicolumn{3}{c}{ ECDF } \\
& $\|$ erro $\|_{2}$ & $\log _{2} r_{e} \approx q$ & Iter & $\|$ erro $\|_{2}$ & $\log _{2} r_{e} \approx q$ & Iter \\
\hline $10 \times 10$ & $2.921 \mathrm{e}-03$ & & 57 & $1.047 \mathrm{e}-06$ & & 44 \\
$20 \times 20$ & $2.261 \mathrm{e}-04$ & 3.691 & 201 & $2.025 \mathrm{e}-08$ & 5.693 & 139 \\
$40 \times 40$ & $1.036 \mathrm{e}-05$ & 4.446 & 465 & $5.026 \mathrm{e}-10$ & 5.332 & 374 \\
$80 \times 80$ & $5.555 \mathrm{e}-07$ & 4.222 & 1614 & $1.514 \mathrm{e}-11$ & 5.053 & 1149 \\
$160 \times 160$ & $3.269 \mathrm{e}-08$ & 4.086 & 5488 & $5.160 \mathrm{e}-13$ & 4.874 & 3767 \\
\hline
\end{tabular}

Tabela 4.1: Erro, ordem de convergência e número de iteraçôes do MDF comparado com o ECDF para o problema de Poisson em uma malha uniforme.

\subsubsection{Teste para malha composta}

A primeira dificuldade que surge é quando são necessários pontos que não pertencem aos nós da malha para calcular algumas derivadas, como pode ser observado na figura (4.1). Essas situações se repetem para o cálculo das outras derivadas envolvidas, deste modo é necessário um método para interpolar os valores de interesse nos pontos que não coincidem com os nós da malha. O método usado para realizar essa interpolação deve ser genérico o suficiente para tratar de quaisquer casos de configurações de células e preciso de modo que não deteriore a ordem de convergência do método numérico.

A metodologia utilizada neste trabalho foi desenvolvida pelo grupo de pesquisa em Mecânica dos Fluidos Computacional do ICMC/USP e está implementada na estrutura de dados Hig-Tree.

Quando algum ponto de interesse $\left(x_{1}, x_{2}, x_{3}\right)$ não coincide com os nós da malha e é necessário avaliar alguma propriedade neste ponto, faz-se uma aproximação deste valor em função dos valores da propriedades em uma nuvem de $m$ pontos $\left(x_{1}^{(1)}, x_{2}^{(1)}, x_{3}^{(1)}\right), \ldots,\left(x_{1}^{(m)}, x_{2}^{(m)}, x_{3}^{(m)}\right)$ próximos ao ponto de interesse. Essa aproximação pode ser feita por um polinômio da seguinte forma

$$
\Phi\left(x_{1}, x_{2}, x_{3}\right)=\sum_{i=1}^{m} \omega_{i} \Phi\left(x_{1}^{(i)}, x_{2}^{(i)}, x_{3}^{(i)}\right),
$$

em que $\Phi\left(x_{1}, x_{2}, x_{3}\right)$ é o valor da propriedade no ponto $\left(x_{1}, x_{2}, x_{3}\right)$ e $\omega_{i}$ é o peso atribuído a cada ponto usado na interpolação. A equação 4.11 pode ser reescrita na seguinte forma matricial

$$
\Phi\left(x_{1}, x_{2}, x_{3}\right)=\omega^{t} b
$$

em que

$$
\begin{gathered}
b=\left(\begin{array}{c}
\Phi\left(x_{1}^{(1)}, x_{2}^{(1)}, x_{3}^{(1)}\right) \\
\vdots \\
\Phi\left(x_{1}^{(m)}, x_{2}^{(m)}, x_{3}^{(m)}\right)
\end{array}\right)= \\
\left(\begin{array}{c}
\left(x_{1}^{(1)}\right)^{i 1}\left(x_{2}^{(1)}\right)^{j 1}\left(x_{3}^{(1)}\right)^{k 1} \cdots\left(x_{1}^{(1)}\right)^{i n}\left(x_{2}^{(1)}\right)^{j n}\left(x_{3}^{(1)}\right)^{k n} \\
\vdots \\
\left(x_{1}^{(m)}\right)^{i 1}\left(x_{2}^{(m)}\right)^{j 1}\left(x_{3}^{(m)}\right)^{k 1} \cdots\left(x_{1}^{(m)}\right)^{i n}\left(x_{2}^{(m)}\right)^{j n}\left(x_{3}^{(m)}\right)^{k n}
\end{array}\right)\left(\begin{array}{c}
\alpha_{1} \\
\vdots \\
\alpha_{n}
\end{array}\right)=A \alpha
\end{gathered}
$$

$A$ é uma matriz $m \times n$. Então

$$
\omega^{t} b=\omega^{t} A \alpha=c^{t} \alpha
$$


para encontrar o vetor de pesos $\omega$ basta resolver o sistema

$$
\omega^{t} A=c^{t} \quad \text { ou seja } \quad A \omega=c
$$

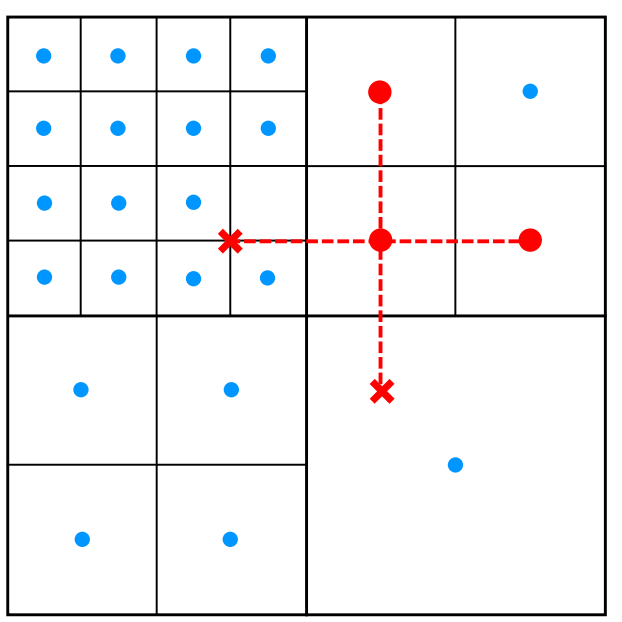

Figura 4.1: Esquema de interpolação

A Hig-Tree tem implementada rotinas para calcular esses pesos $\omega_{i}$ que usam o método dos mínimos quadrados móveis (MLS, do inglês Moving Least Squares) ou o método dos mínimos quadrados (LS, do inglês least squares) para tal. É possível determinar a ordem desejada para interpolação e, como esta depende apenas de uma nuvem de pontos ao redor do ponto de interesse, o formato da malha não influencia na interpolação. Portanto, independente dos níveis de refinamento existentes na vizinhança, a interpolação retornará um valor satisfatório para a propriedade.

Assim sendo, para discretização do problema (4.8), utilizou-se os esquemas de diferenças finitas padrão de quarta ordem comparado como esquema compacto de diferenças finitas de quarta ordem descrito neste trabalho.

Testes para Tolerância variando em $1 e-16,1 e-13,1 e-10$ e $1 e-7$ e ordem do polinômio intepolador variando em 5,4 e 3

Nos testes que fizemos foram analisados dois solvers do PETSc empregando a malha (4.2), obtida de um refinamento feito pela Hig-Tree em três níveis. Escolhemos esta malha porque com ela se tinha um tempo computacional considerável no cálculo da solução do sistema nas contas da Tabela (4.2) (4.3) e (4.4). Os métodos utilizados na solução foram GMRES e BICG (Gradiente Biconjugado), com pré-condicionador ILU(0).

Mostramos os resultados obtidos nas Tabelas (4.2) (4.3) e (4.4) usando tolerância variando em $1 e-16,1 e-13,1 e-10$ e $1 e-7$ e variando a ordem do polinômio interpolador em 5,4 e 3 a fim de experimentalmente analisar com qual ordem os métodos se comportam melhor. 


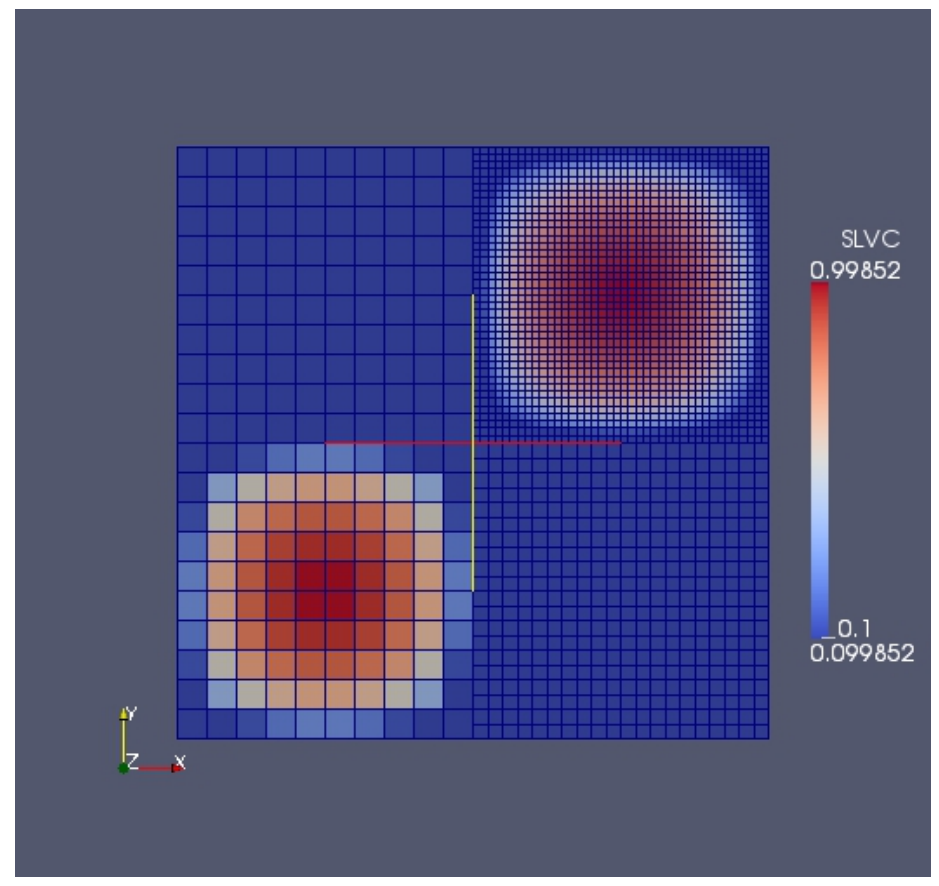

Figura 4.2: Malha correspondente ao testes das tabelas 4.2, 4.3, 4.4

Na figura (4.2), temos a malha composta utilizada na discretização do problema (4.8). Em todos os testes verificou-se ordem de convergência igual a quatro para ambos os métodos, a verificação da ordem de convergência encontra-se no apêndice (A).

\begin{tabular}{c|c|ccc|ccc}
\hline Solver & Tolerância & \multicolumn{3}{|c|}{ MDF } & \multicolumn{3}{c}{ ECDF } \\
& & $\|$ erro $\|_{2}$ & Niter & Time & $\|$ erro $\|_{2}$ & Niter & Time \\
\hline \multirow{5}{*}{ GMRES } & $1 e 10^{-16}$ & $2.513 \mathrm{e}-4$ & 161 & 85 & $2.086 \mathrm{e}-4$ & 82 & 37 \\
& $1 e 10^{-13}$ & $2.513 \mathrm{e}-4$ & 161 & 85 & $2.086 \mathrm{e}-4$ & 82 & 37 \\
& $1 e 10^{-10}$ & $2.513 \mathrm{e}-4$ & 161 & 85 & $2.086 \mathrm{e}-4$ & 82 & 37 \\
& $1 e 10^{-7}$ & $2.513 \mathrm{e}-4$ & 161 & 85 & $2.086 \mathrm{e}-4$ & 82 & 37 \\
\hline \multirow{5}{*}{ BICG } & $1 e 10^{-16}$ & $2.513 \mathrm{e}-4$ & 94 & 66 & $2.086 \mathrm{e}-4$ & 55 & 34 \\
& $1 e 10^{-13}$ & $2.513 \mathrm{e}-4$ & 94 & 66 & $2.086 \mathrm{e}-4$ & 55 & 34 \\
& $1 e 10^{-10}$ & $2.513 \mathrm{e}-4$ & 94 & 66 & $2.086 \mathrm{e}-4$ & 55 & 34 \\
& $1 e 10^{-7}$ & $2.513 \mathrm{e}-4$ & 94 & 66 & $2.086 \mathrm{e}-4$ & 55 & 34 \\
\hline
\end{tabular}

Tabela 4.2: Erro dos métodos MDF de quarta ordem e ECDF de quarta ordem em malha composta com ordem do polinômio interpolador igual a 5. 


\begin{tabular}{c|c|ccc|ccc}
\hline Solver & Tolerância & \multicolumn{3}{|c|}{ MDF } & \multicolumn{3}{c}{ ECDF } \\
& & $\|$ erro $\|_{2}$ & Niter & Time & $\|$ erro $\|_{2}$ & Niter & Time \\
\hline \multirow{3}{*}{ GMRES } & $1 e 10^{-16}$ & $1.044 \mathrm{e}-4$ & 160 & 75 & $1.245 \mathrm{e}-4$ & 82 & 34 \\
& $1 e 10^{-13}$ & $1.044 \mathrm{e}-4$ & 160 & 75 & $1.245 \mathrm{e}-4$ & 82 & 34 \\
& $1 e 10^{-10}$ & $1.044 \mathrm{e}-4$ & 160 & 75 & $1.245 \mathrm{e}-4$ & 82 & 34 \\
& $1 e 10^{-7}$ & $1.044 \mathrm{e}-4$ & 160 & 75 & $1.245 \mathrm{e}-4$ & 82 & 34 \\
\hline \multirow{3}{*}{ BICG } & $1 e 10^{-16}$ & $1.044 \mathrm{e}-4$ & 94 & 57 & $1.245 \mathrm{e}-4$ & 54 & 32 \\
& $1 e 10^{-13}$ & $1.044 \mathrm{e}-4$ & 94 & 57 & $1.245 \mathrm{e}-4$ & 54 & 32 \\
& $1 e 10^{-10}$ & $1.044 \mathrm{e}-4$ & 94 & 57 & $1.245 \mathrm{e}-4$ & 54 & 32 \\
& $1 e 10^{-7}$ & $1.044 \mathrm{e}-4$ & 94 & 57 & $1.245 \mathrm{e}-4$ & 54 & 32 \\
\hline
\end{tabular}

Tabela 4.3: Erro dos métodos MDF de quarta ordem e ECDF de quarta ordem em malha composta com ordem do polinômio interpolador igual a 4 .

\begin{tabular}{c|c|ccc|ccc}
\hline Solver & Tolerância & \multicolumn{3}{|c|}{ MDF } & \multicolumn{3}{c}{ ECDF } \\
& & $\|$ erro $\|_{2}$ & Niter & Time & $\|$ erro $\|_{2}$ & Niter & Time \\
\hline \multirow{3}{*}{ GMRES } & $1 e 10^{-16}$ & $1.946 \mathrm{e}-4$ & 167 & 78 & $2.240 \mathrm{e}-4$ & 82 & 35 \\
& $1 e 10^{-13}$ & $1.946 \mathrm{e}-4$ & 167 & 78 & $2.240 \mathrm{e}-4$ & 82 & 35 \\
& $1 e 10^{-10}$ & $1.946 \mathrm{e}-4$ & 167 & 78 & $2.240 \mathrm{e}-4$ & 82 & 35 \\
& $1 e 10^{-7}$ & $1.946 \mathrm{e}-4$ & 167 & 78 & $2.240 \mathrm{e}-4$ & 82 & 35 \\
\hline \multirow{3}{*}{ BICG } & $1 e 10^{-16}$ & $1.946 \mathrm{e}-4$ & 89 & 54 & $2.240 \mathrm{e}-4$ & 54 & 29 \\
& $1 e 10^{-13}$ & $1.946 \mathrm{e}-4$ & 89 & 54 & $2.240 \mathrm{e}-4$ & 54 & 29 \\
& $1 e 10^{-10}$ & $1.946 \mathrm{e}-4$ & 89 & 54 & $2.240 \mathrm{e}-4$ & 54 & 29 \\
& $1 e 10^{-7}$ & $1.946 \mathrm{e}-4$ & 89 & 54 & $2.240 \mathrm{e}-4$ & 54 & 29 \\
\hline
\end{tabular}

Tabela 4.4: Erro dos métodos MDF de quarta ordem e ECDF de quarta ordem em malha composta com ordem do polinômio interpolador igual a 3.

Analisando as Tabelas (4.2) (4.3) e (4.4) podemos observar que entre os solvers analisados o BICG nos deu resultados mais atrativos pois o tempo computacional e o número de iterações que ele precisa para resolver o sistema é menor comparado com o GMRES.

Na Tabela (4.3) também podemos observar que o erro obtido na solução, quando escolhemos um polinômio interpolador de grau 4, é menor comparado com os resultados para polinômio interpolador de grau 5 (4.2) e de grau 3 (4.4).

Além disso, nos resultados apresentados, verifica-se que o ECQO é mais eficiente que o MDF em todos os casos analisados, apresentando tempo computacional e número de iterações reduzido, praticamente, pela metade quando comparado com o MDF clássico.

\subsection{Problema elíptico com coeficientes variáveis}

Consideramos a equação de Poisson com coeficientes variáveis,

$$
\nabla \cdot(\omega(x, y) \nabla \varphi(x, y))=f(x, y), \quad(x, y) \in \Omega
$$

condições de contorno de Dirichlet, onde $f(x, y)$ é o termo forçante, $\Omega$ é o domínio espacial $[0,1] \times[0,1], \partial \Omega$ é a fronteira do domínio espacial, $\omega(x, y)$ é uma função que será definida nos testes e $\varphi$ é a incógnita.

Empregamos nos testes malha uniforme 4.3 e malha composta com dois niveis de refinamento 4.5. 


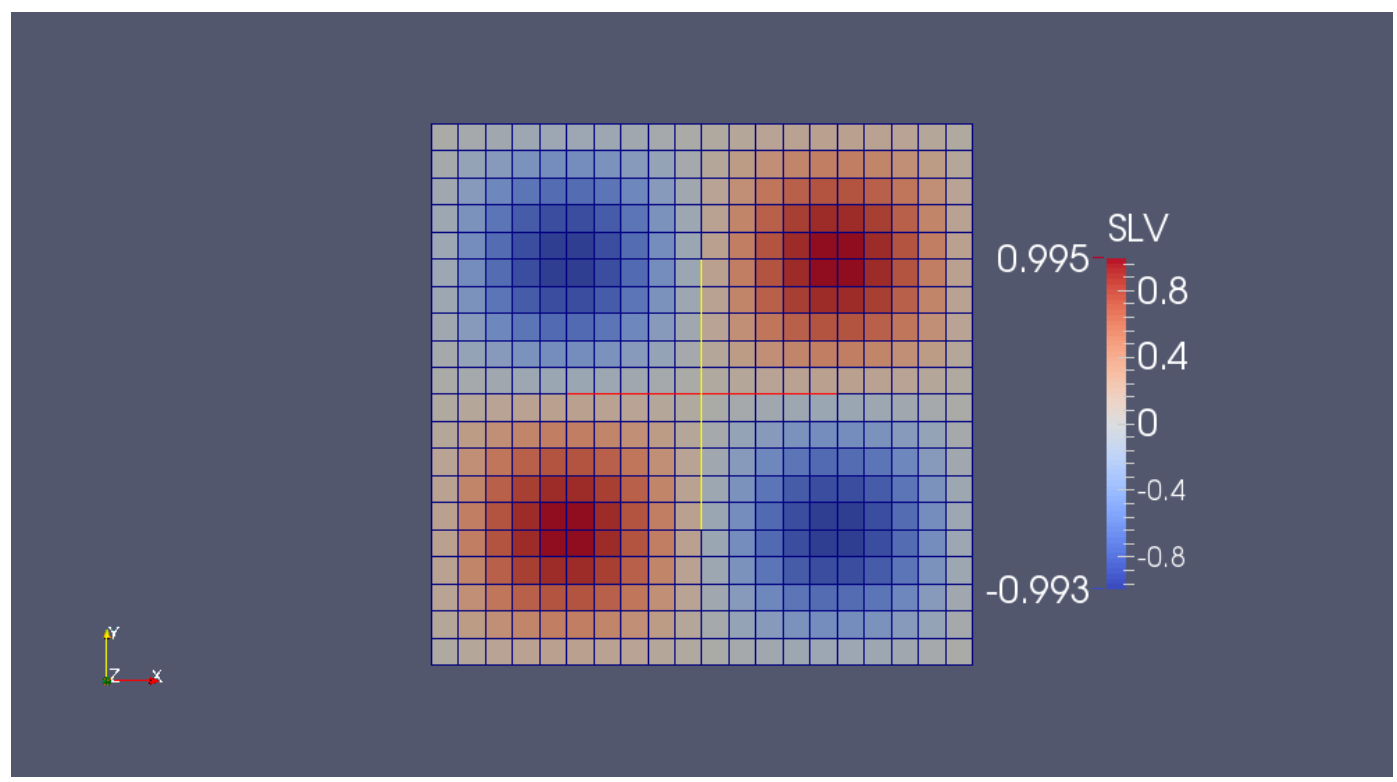

Figura 4.3: Malha uniforme e solução obtida pelo PETSc

\subsubsection{Teste para malha uniforme}

Conforme descrito acima, abordaremos o problema (4.16) com refinamento uniforme na malha (4.3). A vantagem de apresentarmos desta maneira é tornar a exposição de ideias mais simples, didática e respeitar a ordem cronológica real de como as técnicas foram desenvolvidas ao longo do trabalho.

Alguns testes realizados são descritos a seguir, nos quais a solução do sistema linear, dado pela discretização do problema de Poisson (4.16) pelos métodos MDF clássico de quarta ordem e ECQO, foi computado com o uso do PETSc com o método GMRES e BICG com pré-condicionador ILU(0). Adotamos tolerância de $1 e-16$, número máximo de iterações de $10^{4}$ e novamente comparação entre o grau do polinômio interpolador variando em 5,4 e 3 .

As funções usadas para o teste são

$$
\begin{aligned}
\varphi(x, y) & =\sin (2 \pi x) \sin (2 \pi y) \\
f(x, y) & =\omega \nabla^{2} \varphi+\partial_{x} \omega \partial_{x} \varphi+\partial_{y} \omega \partial_{y} \varphi \\
\omega(x, y) & =(1+h)+h \tanh (2 \pi d(x-0.5))
\end{aligned}
$$

onde $h$ controla a variação de $\varphi$ e $d$ a quantidade de células na qual esta variação sucede.

No primeiro teste empregamos $d=2$ e $h=10^{3}$ (com "salto de propriedade"). A condição de contorno de Dirichlet é calculada a partir da solução manufaturada para o problema.

A ordem esperada para a discretização nos dois métodos é quatro, a qual foi comprovada numericamente. Os resultados obtidos estão nas Tabelas (4.5) (4.6) e (4.7).

Testes para Tolerância igual a $1 e-16,1 e-13,1 e-10$ e $1 e-7$ e ordem do polinômio intepolador variando em 5,4 e 3

Nas Tabelas (4.5) (4.6) e (4.7) a coluna "Niter"fornece a quantidade de iterações que os métodos GMRES e BICG, com pré-condicionador ILU(0) do PETSc, precisou para determinar a solução do sistema com precisão $1 e-16,1 e-13,1 e-10$ e $1 e-7$. A coluna "Time"indica o tempo computacional gasto para obter a solução. 


\begin{tabular}{c|c|ccc|ccc}
\hline Solver & Tolerância & \multicolumn{3}{|c|}{ MDF } & \multicolumn{3}{c}{ ECDF } \\
& & $\|$ erro $\|_{2}$ & Niter & Time & $\|$ erro $\|_{2}$ & Niter & Time \\
\hline \multirow{3}{*}{ GMRES } & $1 e 10^{-16}$ & $3.016 \mathrm{e}-4$ & 41 & 6 & $5.301 \mathrm{e}-3$ & 23 & 3 \\
& $1 e 10^{-13}$ & $3.016 \mathrm{e}-4$ & 29 & 6 & $5.301 \mathrm{e}-3$ & 20 & 3 \\
& $1 e 10^{-10}$ & $3.016 \mathrm{e}-4$ & 25 & 4 & $5.301 \mathrm{e}-3$ & 17 & 3 \\
& $1 e 10^{-7}$ & $3.016 \mathrm{e}-4$ & 20 & 4 & $5.301 \mathrm{e}-3$ & 13 & 3 \\
\hline \multirow{3}{*}{ BICG } & $1 e 10^{-16}$ & $3.016 \mathrm{e}-4$ & 36 & 7 & $5.301 \mathrm{e}-3$ & 24 & 4 \\
& $1 e 10^{-13}$ & $3.016 \mathrm{e}-4$ & 32 & 6 & $5.301 \mathrm{e}-3$ & 20 & 4 \\
& $1 e 10^{-10}$ & $3.016 \mathrm{e}-4$ & 26 & 5 & $5.301 \mathrm{e}-3$ & 17 & 3 \\
& $1 e 10^{-7}$ & $3.016 \mathrm{e}-4$ & 21 & 5 & $5.301 \mathrm{e}-3$ & 14 & 3 \\
\hline
\end{tabular}

Tabela 4.5: Erro dos métodos MDF de quarta ordem e ECDF de quarta ordem em malha uniforme com ordem do polinômio interpolador igual a 5.

\begin{tabular}{c|c|ccc|ccc}
\hline Solver & Tolerância & \multicolumn{3}{|c|}{ MDF } & \multicolumn{3}{c}{ ECDF } \\
& & $\|$ erro $\|_{2}$ & Niter & Time & $\|$ erro $\|_{2}$ & Niter & Time \\
\hline \multirow{3}{*}{ GMRES } & $1 e 10^{-16}$ & $2.261 \mathrm{e}-4$ & 42 & 6 & $5.273 \mathrm{e}-3$ & 25 & 3 \\
& $1 e 10^{-13}$ & $2.261 \mathrm{e}-4$ & 31 & 5 & $5.273 \mathrm{e}-3$ & 20 & 3 \\
& $1 e 10^{-10}$ & $2.261 \mathrm{e}-4$ & 25 & 4 & $5.273 \mathrm{e}-3$ & 17 & 3 \\
& $1 e 10^{-7}$ & $2.261 \mathrm{e}-4$ & 20 & 4 & $5.273 \mathrm{e}-3$ & 13 & 2 \\
\hline \multirow{3}{*}{ BICG } & $1 e 10^{-16}$ & $2.261 \mathrm{e}-4$ & 36 & 7 & $5.273 \mathrm{e}-3$ & 24 & 4 \\
& $1 e 10^{-13}$ & $2.261 \mathrm{e}-4$ & 36 & 7 & $5.273 \mathrm{e}-3$ & 24 & 4 \\
& $1 e 10^{-10}$ & $2.261 \mathrm{e}-4$ & 26 & 5 & $5.273 \mathrm{e}-3$ & 17 & 3 \\
& $1 e 10^{-7}$ & $2.261 \mathrm{e}-4$ & 21 & 4 & $5.273 \mathrm{e}-3$ & 14 & 3 \\
\hline
\end{tabular}

Tabela 4.6: Erro dos métodos MDF de quarta ordem e ECDF de quarta ordem em malha uniforme com ordem do polinômio interpolador igual a 4. 


\begin{tabular}{c|c|ccc|ccc}
\hline Solver & Tolerância & \multicolumn{3}{|c|}{ MDF } & \multicolumn{3}{c}{ ECDF } \\
& & $\|$ erro $\|_{2}$ & Niter & Time & $\|$ erro $\|_{2}$ & Niter & Time \\
\hline \multirow{3}{*}{ GMRES } & $1 e 10^{-16}$ & $6.292 \mathrm{e}-4$ & 42 & 6 & $5.211 \mathrm{e}-3$ & 32 & 4 \\
& $1 e 10^{-13}$ & $6.292 \mathrm{e}-4$ & 30 & 4 & $5.211 \mathrm{e}-3$ & 20 & 3 \\
& $1 e 10^{-10}$ & $6.292 \mathrm{e}-4$ & 25 & 4 & $5.211 \mathrm{e}-3$ & 17 & 3 \\
& $1 e 10^{-7}$ & $6.292 \mathrm{e}-4$ & 20 & 3 & $5.211 \mathrm{e}-3$ & 13 & 3 \\
\hline \multirow{3}{*}{ BICG } & $1 e 10^{-16}$ & $6.292 \mathrm{e}-4$ & 36 & 6 & $5.211 \mathrm{e}-3$ & 23 & 4 \\
& $1 e 10^{-13}$ & $6.292 \mathrm{e}-4$ & 32 & 5 & $5.211 \mathrm{e}-3$ & 21 & 4 \\
& $1 e 10^{-10}$ & $6.292 \mathrm{e}-4$ & 26 & 4 & $5.211 \mathrm{e}-3$ & 17 & 3 \\
& $1 e 10^{-7}$ & $6.292 \mathrm{e}-4$ & 21 & 4 & $5.211 \mathrm{e}-3$ & 14 & 2 \\
\hline
\end{tabular}

Tabela 4.7: Erro dos métodos MDF de quarta ordem e ECDF de quarta ordem em malha uniforme com ordem do polinômio interpolador igual a 3.

Analisando as Tabelas (4.5) (4.6) e (4.7) podemos observar o mesmo comparativo feito na secão anterior para problemas com coeficientes constantes. Entre os solvers analisados o BICG nos deu resultados mais atrativos pois o tempo computacional e o número de iterações que ele precisa para resolver o sistema é menor comparado com o GMRES, alem disso verificamos que a variação na tolerancia não interfere nos resultados.

Analisando o grau do polinômio interpolador, aqui também podemos verificar que quando escolhemos um polinômio interpolador de grau 4, ver na Tabela (4.6), o erro obtido na solução é menor comparado com os resultados para polinômio interpolador de grau 5 (4.5) e de grau 3 (4.7).

Apesar de o erro obtido na solução para o ECQO ser maior comparado com o MDF, ainda assim o ECQO é mais eficiente que o MDF em todos os casos analisados, apresentando tempo computacional e número de iterações reduzido, praticamente, pela metade quando comparado com o MDF clássico.

\subsubsection{Teste para malha composta}

Por fim, abordaremos o problema (4.16) com refinamento em malha composta (4.5). A princípio o principal objetivo deste trabalho foi resolver um problema de Poisson com coeficientes variáveis e refinamento em malha composta usando o esquema compacto de quarta ordem e comparar os resultados diretamente com aqueles reportado em [2], porém a metodologia que usamos da biblioteca Hig-Tree difere em algums aspectos da metodologia utilizada por [2] o que dificultou demasiadamente no refino da malha detalhado em [2].

Para este caso o refinamento localizado da malha se deu em particular onde a função $\omega$ definida em (4.16) apresenta elevados gradientes das propriedades próximo à faixa central do domínio de discretização, como podemos observar na Figura (4.4) 


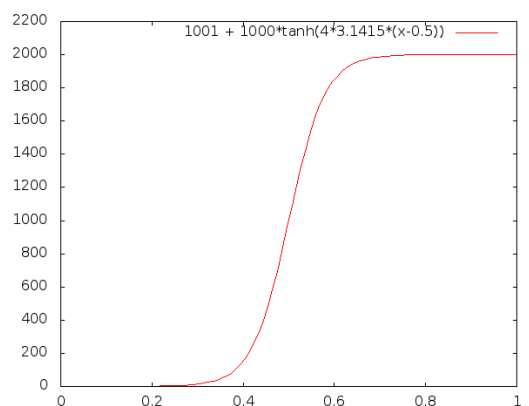

(a) Grafico da função $\omega$

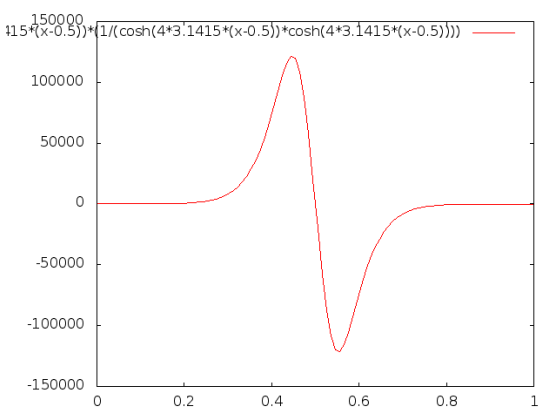

(b) Grafico da função $\frac{\partial \omega}{\partial x}$

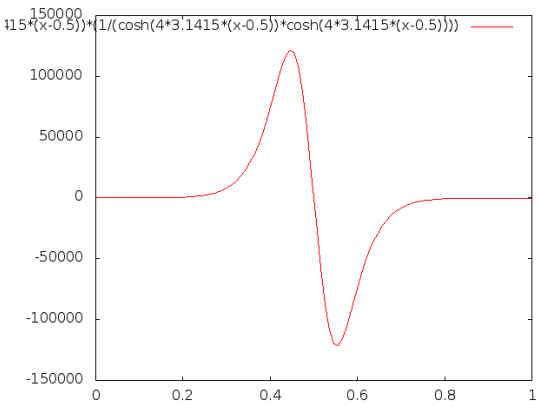

(c) Grafico da função $\frac{\partial^{2} \omega}{\partial x^{2}}$

Figura 4.4: Grafico da função $\omega$

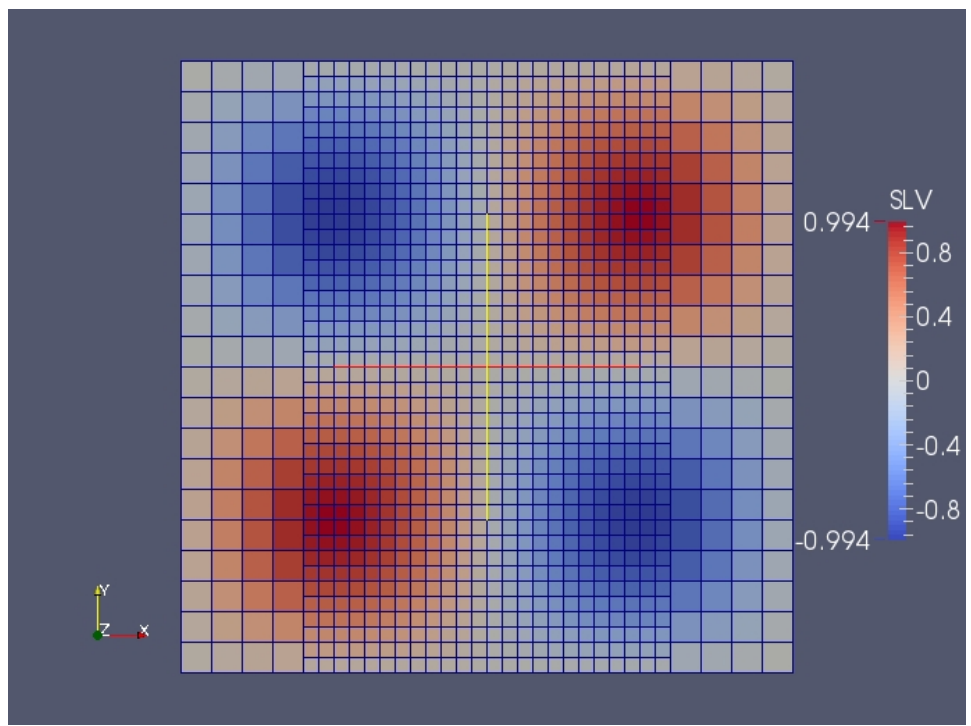

Figura 4.5: Malha composta com dois níveis de refinamento e solução obtida pelo PETSc 
Seguindo o mesmo roteiro de testes descritos nas seções anteriores, nos quais a solução do sistema linear, dado pela discretização do problema de Poisson (4.16) pelos métodos MDF clássico de quarta ordem e ECQO, foi computado com o uso do PETSc com o método GMRES e BICG com pré-condicionador ILU(0). Adotamos a variação da tolerância em $1 e-16,1 e-13,1 e-10$ e $1 e-7$, número máximo de iterações de $10^{4}$ e novamente comparação entre o grau do polinômio interpolador variando em 5,4 e 3 .

As funções usadas para o teste são

$$
\begin{aligned}
\varphi(x, y) & =\sin (2 \pi x) \sin (2 \pi y) \\
f(x, y) & =\omega \nabla^{2} \varphi+\partial_{x} \omega \partial_{x} \varphi+\partial_{y} \omega \partial_{y} \varphi \\
\omega(x, y) & =(1+h)+h \tanh (2 \pi d(x-0.5))
\end{aligned}
$$

onde $h$ controla a variação de $\varphi$ e $d$ a quantidade de células na qual esta variação sucede.

Neste teste, empregamos $d=2$ e $h=10^{3}$ (com "salto de propriedade"). A condição de contorno de Dirichlet é calculada a partir da solução manufaturada para o problema.

A ordem esperada para a discretização nos dois métodos é quatro, a qual foi comprovada numericamente. Os resultados obtidos estão nas Tabelas (4.8) (4.9) e (4.10).

Testes para Tolerância variando em $1 e-16,1 e-13,1 e-10$ e $1 e-7$ e ordem do polinômio intepolador variando em 5,4 e 3

\begin{tabular}{c|c|ccc|ccc}
\hline Solver & Tolerância & \multicolumn{3}{|c|}{ MDF } & \multicolumn{3}{c}{ ECDF } \\
& & $\|$ erro $\|_{2}$ & Niter & Time & $\|$ erro $\|_{2}$ & Niter & Time \\
\hline \multirow{3}{*}{ GMRES } & $1 e 10^{-16}$ & $1.523 \mathrm{e}-4$ & 89 & 31 & $2.367 \mathrm{e}-4$ & 48 & 16 \\
& $1 e 10^{-13}$ & $1.523 \mathrm{e}-4$ & 78 & 27 & $2.367 \mathrm{e}-4$ & 36 & 12 \\
& $1 e 10^{-10}$ & $1.341 \mathrm{e}-4$ & 57 & 21 & $2.367 \mathrm{e}-4$ & 28 & 10 \\
& $1 e 10^{-7}$ & $1.523 \mathrm{e}-4$ & 37 & 18 & $2.367 \mathrm{e}-4$ & 25 & 11 \\
\hline \multirow{3}{*}{ BICG } & $1 e 10^{-16}$ & $1.341 \mathrm{e}-4$ & 59 & 28 & $2.367 \mathrm{e}-4$ & 38 & 15 \\
& $1 e 10^{-13}$ & $1.341 \mathrm{e}-4$ & 54 & 25 & $2.367 \mathrm{e}-4$ & 35 & 14 \\
& $1 e 10^{-10}$ & $1.341 \mathrm{e}-4$ & 48 & 23 & $2.367 \mathrm{e}-4$ & 30 & 12 \\
& $1 e 10^{-7}$ & $1.341 \mathrm{e}-4$ & 43 & 16 & $2.367 \mathrm{e}-4$ & 23 & 8 \\
\hline
\end{tabular}

Tabela 4.8: Erro dos métodos MDF de quarta ordem e ECDF de quarta ordem em malha composta com ordem do polinômio interpolador igual a 5 .

\begin{tabular}{c|c|ccc|ccc}
\hline Solver & Tolerância & \multicolumn{3}{|c|}{ MDF } & \multicolumn{3}{c}{ ECDF } \\
& & $\|$ erro $\|_{2}$ & Niter & Time & $\|$ erro $\|_{2}$ & Niter & Time \\
\hline \multirow{3}{*}{ GMRES } & $1 e 10^{-16}$ & $7.036 \mathrm{e}-5$ & 90 & 28 & $2.447 \mathrm{e}-4$ & 48 & 13 \\
& $1 e 10^{-13}$ & $7.036 \mathrm{e}-5$ & 78 & 24 & $2.447 \mathrm{e}-4$ & 36 & 10 \\
& $1 e 10^{-10}$ & $7.036 \mathrm{e}-5$ & 57 & 18 & $2.447 \mathrm{e}-4$ & 28 & 9 \\
& $1 e 10^{-7}$ & $7.036 \mathrm{e}-5$ & 43 & 14 & $2.447 \mathrm{e}-4$ & 22 & 7 \\
\hline \multirow{3}{*}{ BICG } & $1 e 10^{-16}$ & $7.255 \mathrm{e}-5$ & 63 & 24 & $2.447 \mathrm{e}-4$ & 39 & 13 \\
& $1 e 10^{-13}$ & $7.255 \mathrm{e}-5$ & 54 & 22 & $2.447 \mathrm{e}-4$ & 36 & 13 \\
& $1 e 10^{-10}$ & $7.255 \mathrm{e}-5$ & 48 & 19 & $2.447 \mathrm{e}-4$ & 31 & 11 \\
& $1 e 10^{-7}$ & $7.255 \mathrm{e}-5$ & 37 & 16 & $2.447 \mathrm{e}-4$ & 24 & 9 \\
\hline
\end{tabular}

Tabela 4.9: Erro dos métodos MDF de quarta ordem e ECDF de quarta ordem em malha composta com ordem do polinômio interpolador igual a 4 . 


\begin{tabular}{c|c|ccc|ccc}
\hline Solver & Tolerância & \multicolumn{3}{|c|}{ MDF } & \multicolumn{3}{c}{ ECDF } \\
& & $\|$ erro $\|_{2}$ & Niter & Time & $\|$ erro $\|_{2}$ & Niter & Time \\
\hline \multirow{3}{*}{ GMRES } & $1 e 10^{-16}$ & $1.902 \mathrm{e}-3$ & 84 & 25 & $1.453 \mathrm{e}-3$ & 48 & 14 \\
& $1 e 10^{-13}$ & $1.902 \mathrm{e}-3$ & 79 & 25 & $1.453 \mathrm{e}-3$ & 36 & 11 \\
& $1 e 10^{-10}$ & $1.902 \mathrm{e}-3$ & 57 & 18 & $1.453 \mathrm{e}-3$ & 28 & 8 \\
& $1 e 10^{-7}$ & $1.902 \mathrm{e}-3$ & 43 & 14 & $1.453 \mathrm{e}-3$ & 23 & 7 \\
\hline \multirow{3}{*}{ BICG } & $1 e 10^{-16}$ & $1.902 \mathrm{e}-3$ & 59 & 23 & $1.453 \mathrm{e}-3$ & 41 & 14 \\
& $1 e 10^{-13}$ & $1.902 \mathrm{e}-3$ & 55 & 22 & $1.453 \mathrm{e}-3$ & 35 & 12 \\
& $1 e 10^{-10}$ & $1.902 \mathrm{e}-3$ & 48 & 19 & $1.453 \mathrm{e}-3$ & 30 & 11 \\
& $1 e 10^{-7}$ & $1.902 \mathrm{e}-3$ & 37 & 16 & $1.453 \mathrm{e}-3$ & 25 & 9 \\
\hline
\end{tabular}

Tabela 4.10: Erro dos métodos MDF de quarta ordem e ECDF de quarta ordem em malha composta com ordem do polinômio interpolador igual a 3 .

Nas Tabelas (4.8) (4.9) e (4.10) podemos observar o mesmo comparativo feito na secão anterior quando usamos malha com refinamento uniforme. Observamos em todos os casos que o solver BICG nos deu resultados mais atrativos pois o tempo computacional e o número de iterações que ele precisa para resolver o sistema é menor comparado com o GMRES, além disso verificamos que a variação na tolerância não interferius nos resultados.

Analisando o grau do polinômio interpolador, aqui também podemos verificar que quando escolhemos um polinômio interpolador de grau 4, ver na Tabela (4.9), o erro obtido na solução é menor comparado com os resultados para polinômio interpolador de grau 5 (4.8) e de grau 3 (4.10) tanto no método de diferenças finitas quanto no esquema compacto de diferenças finitas.

Apesar de o erro obtido na solução para o ECQO ser maior comparado com o MDF, ainda assim o ECQO é mais eficiente que o MDF em todos os casos analisados, apresentando tempo computacional e número de iterações reduzido, praticamente, pela metade quando comparado com o MDF clássico. 


\section{Capítulo 5}

\section{Conclusões}

\subsection{Considerações Finais}

O presente trabalho originou-se de uma pesquisa motivada no estudo de métodos numéricos de alta ordem capazes de realizar de maneira robusta e eficiente simulações computacionais de modelos matemáticos clássicos. O método da Projeção de Chorin-Temam aplicado em conjunto com o Esquema Compacto de Diferenças Finitas em malhas hierárquicas, compõem a seleção escolhida para abordar a resolução numérica das equações obtidas do modelo matemático mencionado.

Os problemas-modelos abordados foram discretizados em malha uniforme e em malhas compostas com o uso de uma estrutura de dados chamada Hig-Tree desenvolvida no Instituto de Ciências Matemáticas e Computação, USP São Carlos. Essas discretizações dão origem a sistemas lineares os quais foram resolvidos empregando-se uma biblioteca numérica externa, no caso, o PETSc.

A obtenção de um método de alta ordem compacto no contexto de malhas compostas aplicados à problemas elípticos com coeficientes variáveis ainda é um problema pouco estudado, pois há pouquíssimos relatos a seu respeito. Tal abordagem constitui, em nossa opinião, uma contribuição importante, pois permite, efetivamente, a solução de problemas-modelo essenciais. Além disso, com o uso da Hig-Tree, temos acesso à forma matricial explícita dos sistemas resultantes, dessa forma ganhamos acesso ao uso de vários métodos existentes e disponíveis inclusive em versões paralelas, via uso de bibliotecas numéricas externas como o PETSc. Merece especial destaque que, com as matrizes explicitamente calculadas, podemos usar pré-condicionadores para resolver os sitemas lineares associados de maneira mais eficiente.

\subsection{Sugestões para Pesquisas Futuras}

A pesquisa desenvolvida neste trabalho pode ser enriquecida nos seguintes aspectos:

- Aplicar o esquema compacto de alta ordem em simulações de escoamentos em malhas hierárquicas;

- Implementar modelos computacionais em três dimensões a fim de se obter mais informações sobre o comportamento de um fluido. 
CONCLUSÕES 


\section{Apêndice A \\ Verificação da ordem de convergência}

A.1 Testes referente ao problema (4.8)

A.1.1 Tolerância igual a $1 e-16$ e ordem do polinômio intepolador variando em 3,4 e 5 


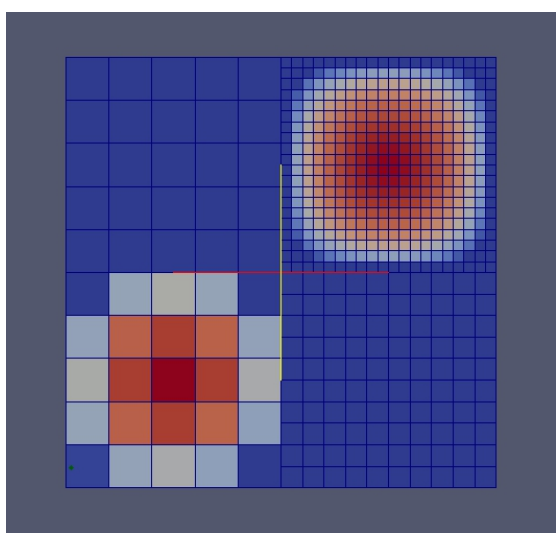

(a)

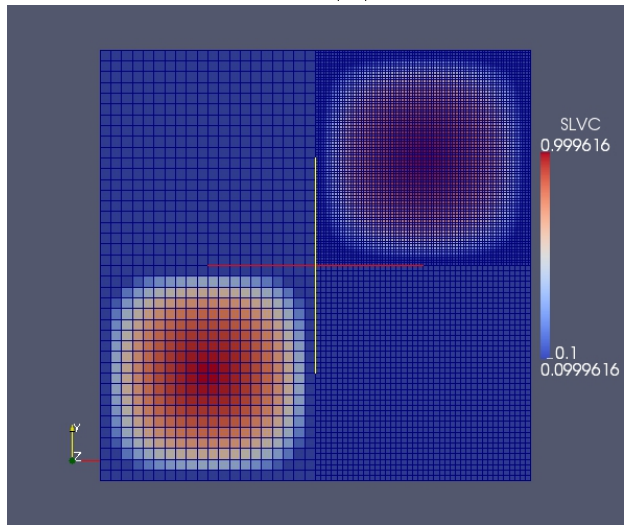

(c)

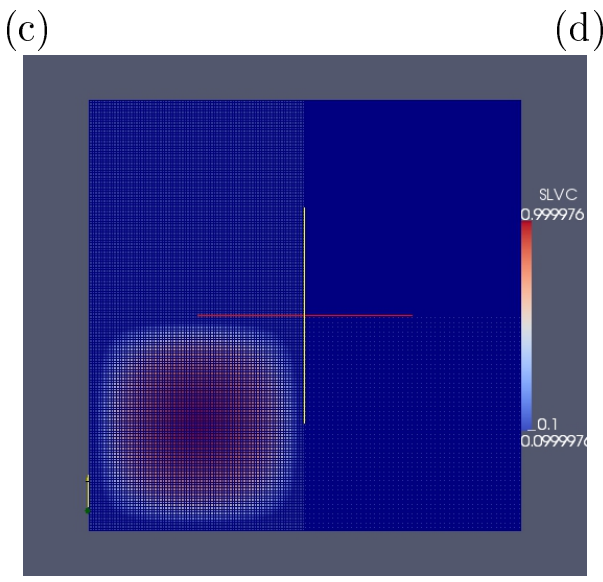

(e)

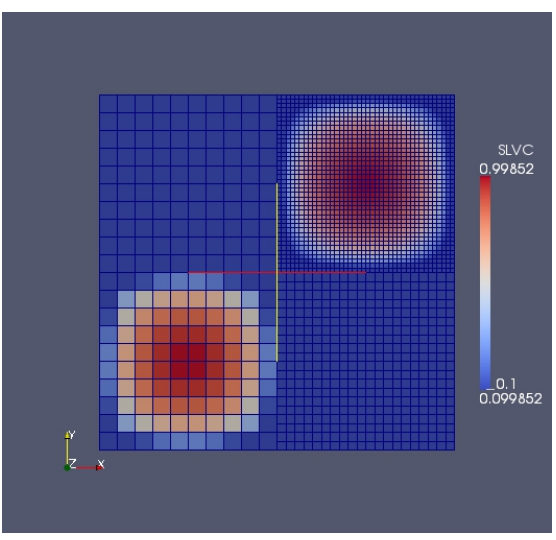

(b)

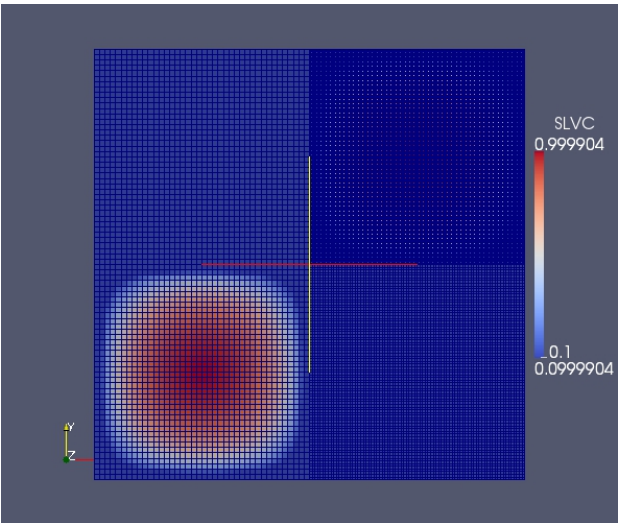

(d)

Figura A.1: (a) Malha G1L3, (b) Malha G2L3, (c) Malha G3L3 e (d) Malha G4L3 e (e) Malha G5L3 


\begin{tabular}{|c|c|c|c|c|c|c|c|c|c|}
\hline $\begin{array}{c}\text { Solver } \\
(\text { tol } 1 e-16)\end{array}$ & Malha & $\|$ erro $\|_{2}$ & $\begin{array}{c}\text { MDF } \\
\log _{2} r_{e} \approx q\end{array}$ & Niter & Time & $\|$ erro $\|_{2}$ & $\begin{array}{c}\mathrm{ECDF} \\
\log _{2} r_{e} \approx q\end{array}$ & Niter & Time \\
\hline \multirow{5}{*}{ GMRES } & G1L3 & $2.960 \mathrm{e}-3$ & & 55 & 11 & $1.895 \mathrm{e}-3$ & & 32 & 6 \\
\hline & G2L3 & $1.946 \mathrm{e}-4$ & 3.927 & 167 & 78 & $2.240 \mathrm{e}-4$ & 3.080 & 82 & 35 \\
\hline & G3L3 & $9.658 \mathrm{e}-6$ & 4.332 & 418 & 643 & $1.338 \mathrm{e}-5$ & 4.064 & 233 & 328 \\
\hline & G4L3 & $6.368 \mathrm{e}-7$ & 3.922 & 100000 & 539624 & $4.839 \mathrm{e}-7$ & 4.790 & 658 & 3375 \\
\hline & G5L3 & $4.867 \mathrm{e}-8$ & 3.709 & 100000 & 2006652 & $1.818 \mathrm{e}-8$ & 4.734 & 100000 & 194956 \\
\hline \multirow{5}{*}{$\mathrm{BICG}$} & G1L3 & $2.960 \mathrm{e}-3$ & & 44 & 12 & $1.895 \mathrm{e}-3$ & & 29 & 6 \\
\hline & G2L3 & $1.946 \mathrm{e}-4$ & 3.927 & 89 & 54 & $2.240 \mathrm{e}-4$ & 3.080 & 54 & 29 \\
\hline & G3L3 & $9.658 \mathrm{e}-6$ & 4.332 & 192 & 365 & $1.338 \mathrm{e}-5$ & 4.064 & 117 & 190 \\
\hline & G4L3 & $6.368 \mathrm{e}-7$ & 3.922 & 397 & 2457 & $4.839 \mathrm{e}-7$ & 4.790 & 230 & 1320 \\
\hline & G5L3 & $4.867 \mathrm{e}-8$ & 3.709 & 828 & 17557 & $1.818 \mathrm{e}-8$ & 4.734 & 494 & 10953 \\
\hline
\end{tabular}

Tabela A.1: Erro e ordem de convergência dos métodos MDF de quarta ordem e ECDF de quarta ordem em malha composta com ordem do polinômio interpolador igual a 3

\begin{tabular}{c|c|cccc|cccc}
\hline $\begin{array}{c}\text { Solver } \\
(\text { tol 1e }-16)\end{array}$ & Malha & \multicolumn{3}{c|}{ MDF } & \multicolumn{3}{c}{ ECDF } \\
& & $\|$ erro $\|_{2}$ & $\log _{2} r_{e} \approx q$ & Niter & Time & $\|$ erro $\|_{2}$ & $\log _{2} r_{e} \approx q$ & Niter & Time \\
& G1L3 & $2.676 \mathrm{e}-3$ & & 55 & 11 & $2.282 \mathrm{e}-3$ & & 33 & 12 \\
GMRES & G2L3 & $1.044 \mathrm{e}-4$ & 4.679 & 160 & 75 & $1.245 \mathrm{e}-4$ & 4.195 & 82 & 34 \\
& G3L3 & $5.982 \mathrm{e}-6$ & 4.125 & 446 & 672 & $6.503 \mathrm{e}-6$ & 4.259 & 233 & 320 \\
& G4L3 & $3.583 \mathrm{e}-7$ & 4.061 & 100000 & 521299 & $3.849 \mathrm{e}-7$ & 4.078 & 716 & 3500 \\
& G5L3 & $2.222 \mathrm{e}-8$ & 4.011 & 100000 & 1924963 & $2.346 \mathrm{e}-8$ & 4.035 & 100000 & 216055 \\
& G1L3 & $2.676 \mathrm{e}-3$ & & 45 & 12 & $2.282 \mathrm{e}-3$ & & 29 & 7 \\
BICG & G2L3 & $1.044 \mathrm{e}-4$ & 4.679 & 94 & 57 & $1.245 \mathrm{e}-4$ & 4.195 & 54 & 32 \\
& G3L3 & $5.982 \mathrm{e}-6$ & 4.125 & 190 & 349 & $6.503 \mathrm{e}-6$ & 4.259 & 115 & 185 \\
& G4L3 & $3.583 \mathrm{e}-7$ & 4.061 & 415 & 2537 & $3.849 \mathrm{e}-7$ & 4.078 & 231 & 1300 \\
& G5L3 & $2.222 \mathrm{e}-8$ & 4.011 & 827 & 18284 & $2.346 \mathrm{e}-8$ & 4.035 & 486 & 10918 \\
\hline
\end{tabular}

Tabela A.2: Erro e ordem de convergência dos métodos MDF de quarta ordem e ECDF de quarta ordem em malha composta com ordem do polinômio interpolador igual a 4 


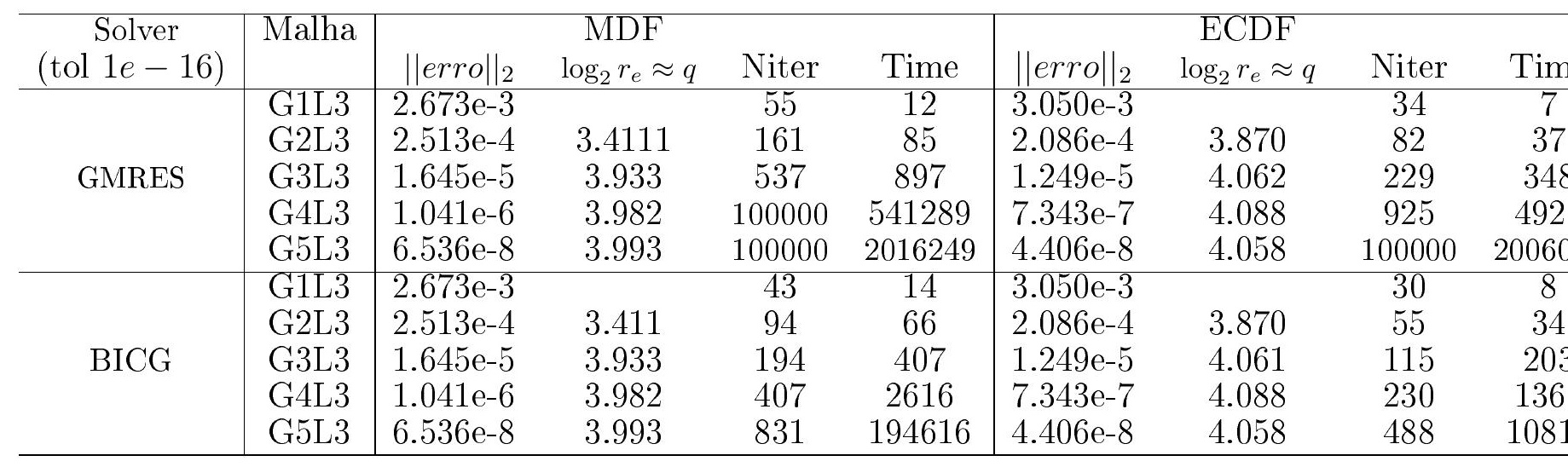

Tabela A.3: Erro e ordem de convergência dos métodos MDF de quarta ordem e ECDF

de quarta ordem em malha composta com ordem do polinômio interpolador igual a 5

\section{A.1.2 Tolerância igual a $1 e-13$ e ordem do polinômio intepolador variando em 3,4 e 5}

\begin{tabular}{c|c|cccc|cccr}
\hline $\begin{array}{c}\text { Solver } \\
(\text { tol } 1 e-13)\end{array}$ & Malha & \multicolumn{3}{c|}{ MDF } & \multicolumn{3}{c}{ ECDF } \\
& & $\|$ erro $\|_{2}$ & $\log _{2} r_{e} \approx q$ & Niter & Time & $\|$ erro $\|_{2}$ & $\log _{2} r_{e} \approx q$ & Niter & Tir \\
& G1L3 & $2.960 \mathrm{e}-3$ & & 55 & 11 & $1.895 \mathrm{e}-3$ & & 32 & 3 \\
GMRES & G2L3 & $1.946 \mathrm{e}-4$ & 3.927 & 167 & 78 & $2.240 \mathrm{e}-4$ & 3.080 & 82 & 32 \\
& G3L3 & $9.658 \mathrm{e}-6$ & 4.332 & 418 & 643 & $1.338 \mathrm{e}-5$ & 4.064 & 233 & 32 \\
& G4L3 & $6.368 \mathrm{e}-7$ & 3.922 & 100000 & 539624 & $4.839 \mathrm{e}-7$ & 4.790 & 658 & 33 \\
& G5L3 & $4.867 \mathrm{e}-8$ & 3.709 & 100000 & 2006652 & $1.818 \mathrm{e}-8$ & 4.734 & 100000 & 1949 \\
& G1L3 & $2.960 \mathrm{e}-3$ & & 44 & 12 & $1.895 \mathrm{e}-3$ & & 29 & 6 \\
BICG & G2L3 & $1.946 \mathrm{e}-4$ & 3.927 & 89 & 54 & $2.240 \mathrm{e}-4$ & 3.080 & 54 & 117 \\
& G3L3 & $9.658 \mathrm{e}-6$ & 4.332 & 192 & 365 & $1.338 \mathrm{e}-5$ & 4.064 & 117 \\
& G4L3 & $6.368 \mathrm{e}-7$ & 3.922 & 397 & 2457 & $4.839 \mathrm{e}-7$ & 4.790 & 230 & 13 \\
& G5L3 & $4.867 \mathrm{e}-8$ & 3.709 & 828 & 17557 & $1.818 \mathrm{e}-8$ & 4.734 & 494 & 109 \\
\hline
\end{tabular}

Tabela A.4: Erro e ordem de convergência dos métodos MDF de quarta ordem e ECDF de quarta ordem em malha composta com ordem do polinômio interpolador igual a 3 


\begin{tabular}{|c|c|c|c|c|c|c|c|c|c|}
\hline $\begin{array}{c}\text { Solver } \\
\text { (tol } 1 e-13)\end{array}$ & Malha & $\|$ erro $\|_{2}$ & $\begin{array}{c}\text { MDF } \\
\log _{2} r_{e} \approx q\end{array}$ & Niter & Time & $\|$ erro $\|_{2}$ & $\begin{array}{c}\mathrm{ECDF} \\
\log _{2} r_{e} \approx q\end{array}$ & Niter & Time \\
\hline \multirow{5}{*}{ GMRES } & G1L3 & $2.676 \mathrm{e}-3$ & & 55 & 11 & $2.282 \mathrm{e}-3$ & & 33 & 12 \\
\hline & G2L3 & $1.044 \mathrm{e}-4$ & 4.679 & 160 & 75 & $1.245 \mathrm{e}-4$ & 4.195 & 82 & 34 \\
\hline & G3L3 & $5.982 \mathrm{e}-6$ & 4.125 & 446 & 672 & $6.503 \mathrm{e}-6$ & 4.259 & 233 & 320 \\
\hline & G4L3 & $3.583 \mathrm{e}-7$ & 4.061 & 100000 & 521299 & $3.849 \mathrm{e}-7$ & 4.078 & 716 & 3500 \\
\hline & G5L3 & $2.222 \mathrm{e}-8$ & 4.011 & 100000 & 1924963 & $2.346 \mathrm{e}-8$ & 4.035 & 100000 & 216055 \\
\hline \multirow{5}{*}{$\mathrm{BICG}$} & G1L3 & $2.676 \mathrm{e}-3$ & & 45 & 12 & $2.282 \mathrm{e}-3$ & & 29 & 7 \\
\hline & G2L3 & $1.044 \mathrm{e}-4$ & 4.679 & 94 & 57 & $1.245 \mathrm{e}-4$ & 4.195 & 54 & 32 \\
\hline & G3L3 & $5.982 \mathrm{e}-6$ & 4.125 & 190 & 349 & $6.503 \mathrm{e}-6$ & 4.259 & 115 & 185 \\
\hline & G4L3 & $3.583 \mathrm{e}-7$ & 4.061 & 415 & 2537 & $3.849 \mathrm{e}-7$ & 4.078 & 231 & 1300 \\
\hline & G5L3 & $2.222 \mathrm{e}-8$ & 4.011 & 827 & 18284 & $2.346 \mathrm{e}-8$ & 4.035 & 486 & 10918 \\
\hline
\end{tabular}

Tabela A.5: Erro e ordem de convergência dos métodos MDF de quarta ordem e ECDF de quarta ordem em malha composta com ordem do polinômio interpolador igual a 4

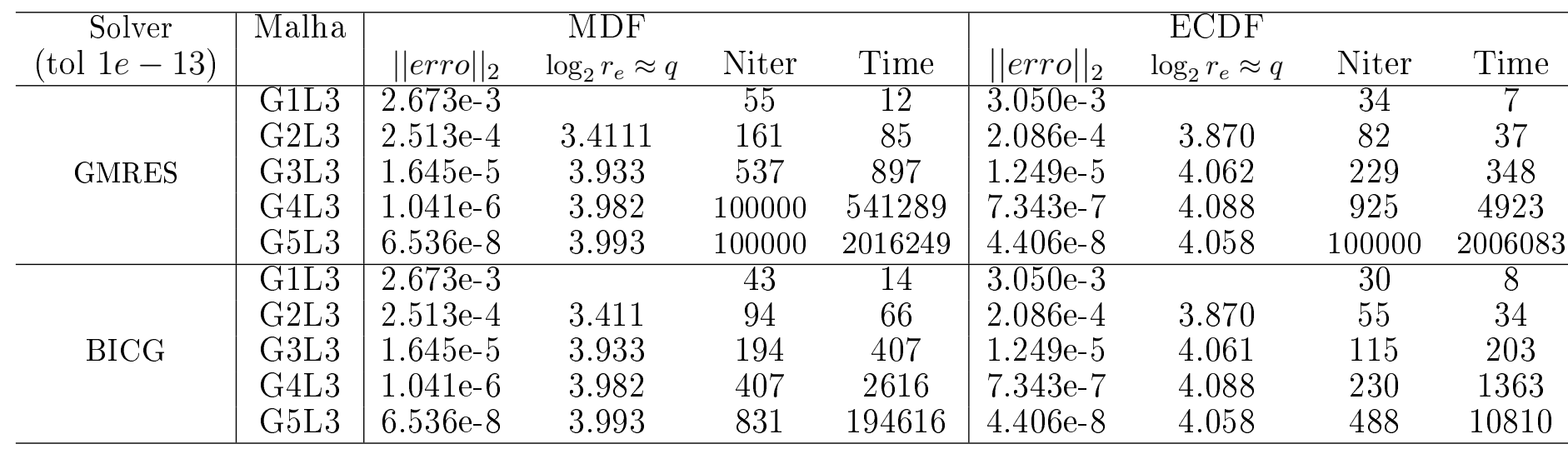

Tabela A.6: Erro e ordem de convergência dos métodos MDF de quarta ordem e ECDF de quarta ordem em malha composta com ordem do polinômio interpolador igual a 5

\section{A.1.3 Tolerância igual a $1 e-10$ e ordem do polinômio intepolador variando em 3,4 e 5}




\begin{tabular}{|c|c|c|c|c|c|c|c|c|c|}
\hline \multirow{2}{*}{$\begin{array}{c}\text { Solver } \\
\text { (tol } 1 e-10)\end{array}$} & \multirow[t]{2}{*}{ Malha } & \multicolumn{3}{|c|}{ MDF } & & \multicolumn{4}{|c|}{ ECDF } \\
\hline & & $\|$ erro $\|_{2}$ & $\log _{2} r_{e} \approx q$ & Niter & Time & $\left\|_{\text {erro }}\right\|_{2}$ & $\log _{2} r_{e} \approx q$ & Niter & Tir \\
\hline \multirow{5}{*}{ GMRES } & G1L3 & $2.960 \mathrm{e}-3$ & & 55 & 11 & $1.895 \mathrm{e}-3$ & & 32 & \\
\hline & G2L3 & $1.946 \mathrm{e}-4$ & 3.927 & 167 & 78 & $2.240 \mathrm{e}-4$ & 3.080 & 82 & \\
\hline & G3L3 & $9.658 \mathrm{e}-6$ & 4.332 & 418 & 643 & $1.338 \mathrm{e}-5$ & 4.064 & 233 & \\
\hline & G4L3 & $6.368 \mathrm{e}-7$ & 3.922 & 100000 & 539624 & $4.839 \mathrm{e}-7$ & 4.790 & 658 & 33 \\
\hline & G5L3 & $4.867 \mathrm{e}-8$ & 3.709 & 100000 & 2006652 & $1.818 \mathrm{e}-8$ & 4.734 & 100000 & 1949 \\
\hline \multirow{5}{*}{$\mathrm{BICG}$} & G1L3 & $2.960 \mathrm{e}-3$ & & 44 & 12 & $1.895 \mathrm{e}-3$ & & 29 & \\
\hline & G2L3 & $1.946 \mathrm{e}-4$ & 3.927 & 89 & 54 & $2.240 \mathrm{e}-4$ & 3.080 & 54 & \\
\hline & G3L3 & $9.658 \mathrm{e}-6$ & 4.332 & 192 & 365 & $1.338 \mathrm{e}-5$ & 4.064 & 117 & \\
\hline & G4L3 & $6.368 \mathrm{e}-7$ & 3.922 & 397 & 2457 & $4.839 \mathrm{e}-7$ & 4.790 & 230 & \\
\hline & G5L3 & $4.867 \mathrm{e}-8$ & 3.709 & 828 & 17557 & $1.818 \mathrm{e}-8$ & 4.734 & 494 & \\
\hline
\end{tabular}

Tabela A.7: Erro e ordem de convergência dos métodos MDF de quarta ordem e ECDF de quarta ordem em malha composta com ordem do polinômio interpolador igual a 3

\begin{tabular}{|c|c|c|c|c|c|c|c|c|c|}
\hline Solver & Malha & & MDF & & & & ECDF & & \\
\hline$($ tol $1 e-10)$ & & $\|$ erro $\|_{2}$ & $\log _{2} r_{e} \approx q$ & Niter & Time & $\|$ erro $\|_{2}$ & $\log _{2} r_{e} \approx q$ & Niter & Tir \\
\hline \multirow{5}{*}{ GMRES } & G1L3 & $2.676 \mathrm{e}-3$ & & 55 & 11 & $2.282 \mathrm{e}-3$ & & 33 & \\
\hline & G2L3 & $1.044 \mathrm{e}-4$ & 4.679 & 160 & 75 & $1.245 \mathrm{e}-4$ & 4.195 & 82 & \\
\hline & G3L3 & $5.982 \mathrm{e}-6$ & 4.125 & 446 & 672 & $6.503 \mathrm{e}-6$ & 4.259 & 233 & \\
\hline & G4L3 & $3.583 \mathrm{e}-7$ & 4.061 & 100000 & 521299 & $3.849 \mathrm{e}-7$ & 4.078 & 716 & \\
\hline & G5L3 & $2.222 \mathrm{e}-8$ & 4.011 & 100000 & 1924963 & $2.346 \mathrm{e}-8$ & 4.035 & 100000 & \\
\hline \multirow{5}{*}{ BICG } & G1L3 & $2.676 \mathrm{e}-3$ & & 45 & 12 & $2.282 \mathrm{e}-3$ & & 29 & \\
\hline & G2L3 & $1.044 \mathrm{e}-4$ & 4.679 & 94 & 57 & $1.245 \mathrm{e}-4$ & 4.195 & 54 & \\
\hline & G3L3 & $5.982 \mathrm{e}-6$ & 4.125 & 190 & 349 & $6.503 \mathrm{e}-6$ & 4.259 & 115 & \\
\hline & G4L3 & $3.583 \mathrm{e}-7$ & 4.061 & 415 & 2537 & $3.849 \mathrm{e}-7$ & 4.078 & 231 & \\
\hline & G5L3 & $2.222 \mathrm{e}-8$ & 4.011 & 827 & 18284 & $2.346 \mathrm{e}-8$ & 4.035 & 486 & \\
\hline
\end{tabular}

Tabela A.8: Erro e ordem de convergência dos métodos MDF de quarta ordem e ECDF de quarta ordem em malha composta com ordem do polinômio interpolador igual a 4 


\begin{tabular}{|c|c|c|c|c|c|c|c|c|c|}
\hline \multirow{2}{*}{$\begin{array}{c}\text { Solver } \\
(\text { tol } 1 e-10)\end{array}$} & \multirow[t]{2}{*}{ Malha } & \multicolumn{3}{|c|}{ MDF } & \multicolumn{5}{|c|}{ ECDF } \\
\hline & & $\|$ erro $\|_{2}$ & $\log _{2} r_{e} \approx q$ & Niter & Time & $\|$ erro $\|_{2}$ & $\log _{2} r_{e} \approx q$ & Niter & Time \\
\hline \multirow{5}{*}{ GMRES } & G1L3 & $2.673 \mathrm{e}-3$ & & 55 & 12 & $3.050 \mathrm{e}-3$ & & 34 & 7 \\
\hline & G2L3 & $2.513 \mathrm{e}-4$ & 3.4111 & 161 & 85 & $2.086 \mathrm{e}-4$ & 3.870 & 82 & 37 \\
\hline & G3L3 & $1.645 \mathrm{e}-5$ & 3.933 & 537 & 897 & $1.249 \mathrm{e}-5$ & 4.062 & 229 & 348 \\
\hline & G4L3 & $1.041 \mathrm{e}-6$ & 3.982 & 100000 & 541289 & $7.343 \mathrm{e}-7$ & 4.088 & 925 & 4923 \\
\hline & G5L3 & $6.536 \mathrm{e}-8$ & 3.993 & 100000 & 2016249 & $4.406 \mathrm{e}-8$ & 4.058 & 100000 & 2006083 \\
\hline \multirow{5}{*}{$\mathrm{BICG}$} & G1L3 & $2.673 \mathrm{e}-3$ & & 43 & 14 & $3.050 \mathrm{e}-3$ & & 30 & 8 \\
\hline & G2L3 & $2.513 \mathrm{e}-4$ & 3.411 & 94 & 66 & $2.086 \mathrm{e}-4$ & 3.870 & 55 & 34 \\
\hline & G3L3 & $1.645 \mathrm{e}-5$ & 3.933 & 194 & 407 & $1.249 \mathrm{e}-5$ & 4.061 & 115 & 203 \\
\hline & G4L3 & $1.041 \mathrm{e}-6$ & 3.982 & 407 & 2616 & $7.343 \mathrm{e}-7$ & 4.088 & 230 & 1363 \\
\hline & G5L3 & $6.536 \mathrm{e}-8$ & 3.993 & 831 & 194616 & $4.406 \mathrm{e}-8$ & 4.058 & 488 & 10810 \\
\hline
\end{tabular}

Tabela A.9: Erro e ordem de convergência dos métodos MDF de quarta ordem e ECDF de quarta ordem em malha composta com ordem do polinômio interpolador igual a 5

\section{A.1.4 Tolerância igual a $1 e-7$ e ordem do polinômio intepolador variando em 3,4 e 5}

\begin{tabular}{c|l|cccc|cccc}
\hline $\begin{array}{c}\text { Solver } \\
\text { (tol } 1 e-7)\end{array}$ & Malha & \multicolumn{3}{c|}{ MDF } & \multicolumn{3}{c}{ ECDF } \\
& & $\|$ erro $\|_{2}$ & $\log _{2} r_{e} \approx q$ & Niter & Time & $\|$ erro $\|_{2}$ & $\log _{2} r_{e} \approx q$ & Niter & Time \\
\hline \multirow{5}{*}{ GMRES } & G1L3 & $2.960 \mathrm{e}-3$ & & 55 & 11 & $1.895 \mathrm{e}-3$ & & 32 & 6 \\
& G2L3 & $1.946 \mathrm{e}-4$ & 3.927 & 167 & 78 & $2.240 \mathrm{e}-4$ & 3.080 & 82 & 35 \\
& G3L3 & $9.658 \mathrm{e}-6$ & 4.332 & 418 & 643 & $1.338 \mathrm{e}-5$ & 4.064 & 233 & 328 \\
& G4L3 & $6.368 \mathrm{e}-7$ & 3.922 & 100000 & 539624 & $4.839 \mathrm{e}-7$ & 4.790 & 658 & 3375 \\
& G5L3 & $4.867 \mathrm{e}-8$ & 3.709 & 100000 & 2006652 & $1.818 \mathrm{e}-8$ & 4.734 & 100000 & 1949565 \\
& G1L3 & $2.960 \mathrm{e}-3$ & & 44 & 12 & $1.895 \mathrm{e}-3$ & & 29 & 6 \\
BICG & G2L3 & $1.946 \mathrm{e}-4$ & 3.927 & 89 & 54 & $2.240 \mathrm{e}-4$ & 3.080 & 54 & 29 \\
& G3L3 & $9.658 \mathrm{e}-6$ & 4.332 & 192 & 365 & $1.338 \mathrm{e}-5$ & 4.064 & 117 & 190 \\
& G4L3 & $6.368 \mathrm{e}-7$ & 3.922 & 397 & 2457 & $4.839 \mathrm{e}-7$ & 4.790 & 230 & 1320 \\
& G5L3 & $4.867 \mathrm{e}-8$ & 3.709 & 828 & 17557 & $1.818 \mathrm{e}-8$ & 4.734 & 494 & 10953 \\
\hline
\end{tabular}

Tabela A.10: Erro e ordem de convergência dos métodos MDF de quarta ordem e ECDF de quarta ordem em malha composta com ordem do polinômio interpolador igual a 3 


\begin{tabular}{|c|c|c|c|c|c|c|c|c|c|}
\hline \multirow{2}{*}{$\begin{array}{c}\text { Solver } \\
\text { (tol } 1 e-7)\end{array}$} & \multirow[t]{2}{*}{ Malha } & \multicolumn{3}{|c|}{ MDF } & & \multicolumn{4}{|c|}{ ECDF } \\
\hline & & $\|$ erro $\|_{2}$ & $\log _{2} r_{e} \approx q$ & Niter & Time & $\|$ erro $\|_{2}$ & $\log _{2} r_{e} \approx q$ & Niter & Tim \\
\hline \multirow{5}{*}{ GMRES } & G1L3 & $2.676 \mathrm{e}-3$ & & 55 & 11 & $2.282 \mathrm{e}-3$ & & 33 & 12 \\
\hline & G2L3 & $1.044 \mathrm{e}-4$ & 4.679 & 160 & 75 & $1.245 \mathrm{e}-4$ & 4.195 & 82 & 34 \\
\hline & G3L3 & $5.982 \mathrm{e}-6$ & 4.125 & 446 & 672 & $6.503 \mathrm{e}-6$ & 4.259 & 233 & 320 \\
\hline & G4L3 & $3.583 \mathrm{e}-7$ & 4.061 & 100000 & 521299 & $3.849 \mathrm{e}-7$ & 4.078 & 716 & \\
\hline & G5L3 & $2.222 \mathrm{e}-8$ & 4.011 & 100000 & 1924963 & $2.346 \mathrm{e}-8$ & 4.035 & 100000 & 21605 \\
\hline \multirow{5}{*}{$\mathrm{BICG}$} & G1L3 & $2.676 \mathrm{e}-3$ & & 45 & 12 & $2.282 \mathrm{e}-3$ & & 29 & 7 \\
\hline & G2L3 & $1.044 \mathrm{e}-4$ & 4.679 & 94 & 57 & $1.245 \mathrm{e}-4$ & 4.195 & 54 & 32 \\
\hline & G3L3 & $5.982 \mathrm{e}-6$ & 4.125 & 190 & 349 & $6.503 \mathrm{e}-6$ & 4.259 & 115 & 185 \\
\hline & G4L3 & $3.583 \mathrm{e}-7$ & 4.061 & 415 & 2537 & $3.849 \mathrm{e}-7$ & 4.078 & 231 & 130 \\
\hline & G5L3 & $2.222 \mathrm{e}-8$ & 4.011 & 827 & 18284 & $2.346 \mathrm{e}-8$ & 4.035 & 486 & 109 \\
\hline
\end{tabular}

Tabela A.11: Erro e ordem de convergência dos métodos MDF de quarta ordem e ECDF de quarta ordem em malha composta com ordem do polinômio interpolador igual a 4

\begin{tabular}{c|c|cccc|cccc}
\hline $\begin{array}{c}\text { Solver } \\
(\text { tol } 1 e-7)\end{array}$ & Malha & \multicolumn{3}{c|}{ MDF } & \multicolumn{3}{c}{ ECDF } \\
& & $\|$ erro $\|_{2}$ & $\log _{2} r_{e} \approx q$ & Niter & Time & $\|$ erro $\|_{2}$ & $\log _{2} r_{e} \approx q$ & Niter & Time \\
& G1L3 & $2.673 \mathrm{e}-3$ & & 55 & 12 & $3.050 \mathrm{e}-3$ & & 34 & 7 \\
GMRES & G2L3 & $2.513 \mathrm{e}-4$ & 3.4111 & 161 & 85 & $2.086 \mathrm{e}-4$ & 3.870 & 82 & 37 \\
& G3L3 & $1.645 \mathrm{e}-5$ & 3.933 & 537 & 897 & $1.249 \mathrm{e}-5$ & 4.062 & 229 & 348 \\
& G4L3 & $1.041 \mathrm{e}-6$ & 3.982 & 100000 & 541289 & $7.343 \mathrm{e}-7$ & 4.088 & 925 & 4923 \\
& G5L3 & $6.536 \mathrm{e}-8$ & 3.993 & 100000 & 2016249 & $4.406 \mathrm{e}-8$ & 4.058 & 100000 & 200608 \\
\hline \multirow{5}{*}{ BICG } & G1L3 & $2.673 \mathrm{e}-3$ & & 43 & 14 & $3.050 \mathrm{e}-3$ & & 30 & 8 \\
& G2L3 & $2.513 \mathrm{e}-4$ & 3.411 & 94 & 66 & $2.086 \mathrm{e}-4$ & 3.870 & 55 & 34 \\
& G3L3 & $1.645 \mathrm{e}-5$ & 3.933 & 194 & 407 & $1.249 \mathrm{e}-5$ & 4.061 & 115 & 203 \\
& G4L3 & $1.041 \mathrm{e}-6$ & 3.982 & 407 & 2616 & $7.343 \mathrm{e}-7$ & 4.088 & 230 & 1363 \\
& G5L3 & $6.536 \mathrm{e}-8$ & 3.993 & 831 & 194616 & $4.406 \mathrm{e}-8$ & 4.058 & 488 & 10810 \\
\hline
\end{tabular}

Tabela A.12: Erro e ordem de convergência dos métodos MDF de quarta ordem e ECDF de quarta ordem em malha composta com ordem do polinômio interpolador igual a 5

\section{A.2 Testes referente ao problemas (4.16)}

\section{A.2.1 Tolerância igual a $1 e-16$ e ordem do polinômio intepolador variando em 3,4 e 5}




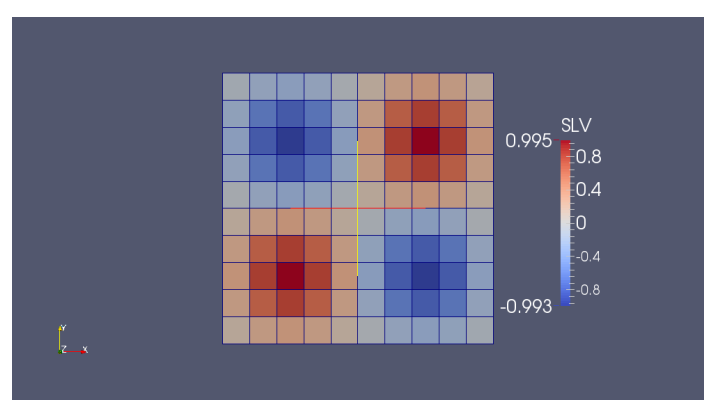

(a)

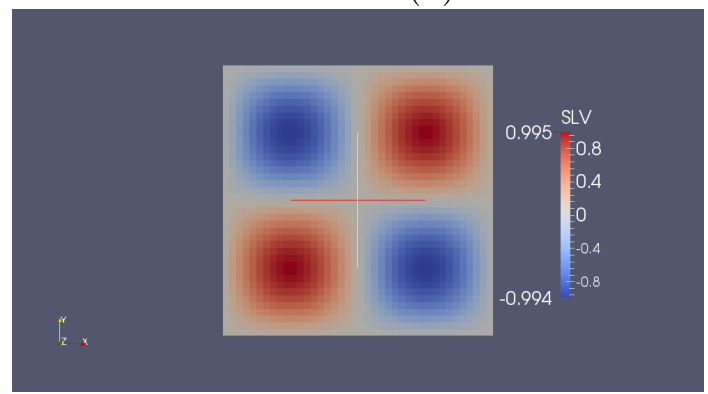

(c)

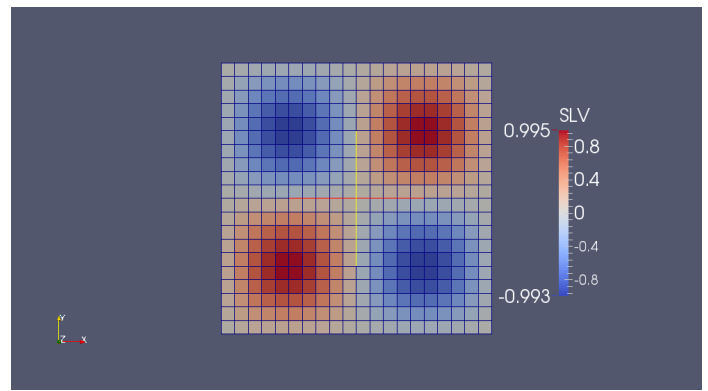

(b)

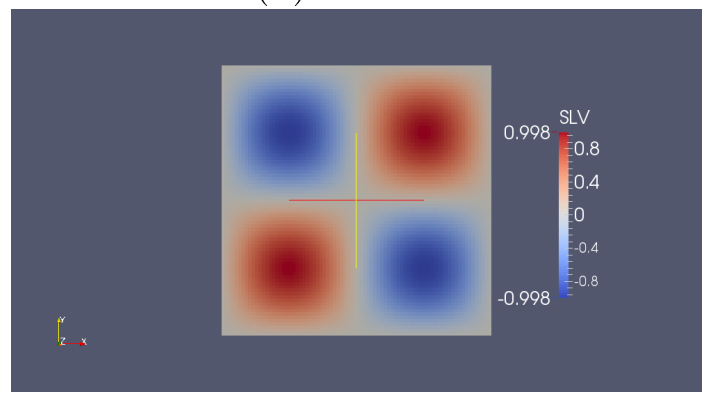

(d)

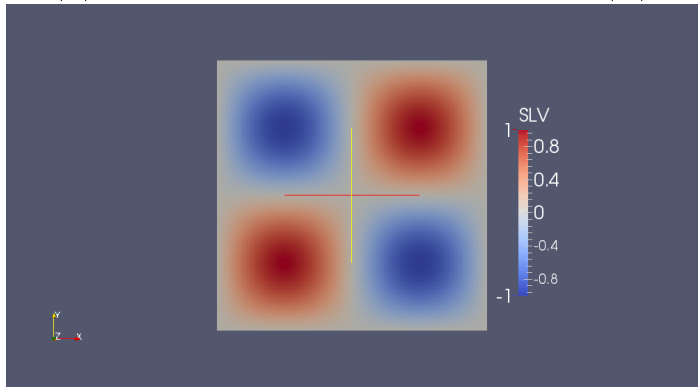

(e)

Figura A.2: (a) Malha G1L1, (b) Malha G2L1, (c) Malha G3L1 e (d) Malha G4L1 e (e) Malha G5L1

\begin{tabular}{c|l|cccc|cccc}
\hline $\begin{array}{c}\text { Solver } \\
(\text { tol } 1 e-16)\end{array}$ & Malha & \multicolumn{3}{c|}{ MDF } & \multicolumn{4}{c}{ ECDF } \\
$\log _{2} r_{e} \approx q$ & Niter & Time & $\|$ erro $\|_{2}$ & $\log _{2} r_{e} \approx q$ & Niter & Time \\
\hline \multirow{5}{*}{ GMRES } & G1L1 & $8.051 \mathrm{e}-3$ & & 23 & 3 & $2.500 \mathrm{e}-1$ & & 31 & 2 \\
& G2L1 & $6.292 \mathrm{e}-4$ & 3.677 & 42 & 6 & $5.211 \mathrm{e}-3$ & 4.564 & 32 & 4 \\
& G3L3 & $3.411 \mathrm{e}-5$ & 4.204 & 110 & 33 & $2.201 \mathrm{e}-4$ & 4.564 & 52 & 16 \\
& G4L1 & $2.215 \mathrm{e}-6$ & 3.944 & 295 & 291 & $1.250 \mathrm{e}-5$ & 4.137 & 139 & 131 \\
& G5L1 & $1.426 \mathrm{e}-7$ & 3.956 & 100000 & 365172 & $7.638 \mathrm{e}-7$ & 4.033 & 1109 & 3964 \\
\hline \multirow{5}{*}{ BICG } & G1L1 & $8.051-3$ & & 20 & 2 & $2.500 \mathrm{e}-1$ & & 15 & 2 \\
& G2L1 & $6.292 \mathrm{e}-4$ & 3.677 & 36 & 6 & $5.211 \mathrm{e}-3$ & 5.584 & 23 & 4 \\
& G3L3 & $3.411 \mathrm{e}-5$ & 4.204 & 71 & 26 & $2.201 \mathrm{e}-4$ & 4.564 & 43 & 14 \\
& G4L1 & $2.215 \mathrm{e}-6$ & 3.944 & 138 & 154 & $1.250 \mathrm{e}-5$ & 4.137 & 81 & 85 \\
& G5L1 & $1.426 \mathrm{e}-7$ & 3.956 & 299 & 1168 & $7.638 \mathrm{e}-7$ & 4.033 & 153 & 587 \\
\hline
\end{tabular}

Tabela A.13: Erro e ordem de convergência dos métodos MDF de quarta ordem e ECDF de quarta ordem em malha uniforme com ordem do polinômio interpolador igual a 3 


\begin{tabular}{c|c|cccc|cccc}
\hline $\begin{array}{c}\text { Solver } \\
\text { tol 1e-16) }\end{array}$ & Malha & \multicolumn{3}{|c|}{ MDF } & \multicolumn{3}{c}{ ECDF } \\
& & $\|$ erro $\|_{2}$ & $\log _{2} r_{e} \approx q$ & Niter & Time & $\|$ erro $\|_{2}$ & $\log _{2} r_{e} \approx q$ & Niter & Time \\
\hline \multirow{5}{*}{ GMRES } & G1L1 & $2.921 \mathrm{e}-3$ & & 20 & 2 & $2.519 \mathrm{e}-1$ & & 19 & 2 \\
& G2L1 & $2.261 \mathrm{e}-4$ & 3.691 & 42 & 6 & $5.273 \mathrm{e}-3$ & 5.578 & 25 & 3 \\
& G3L3 & $1.036 \mathrm{e}-5$ & 4.446 & 107 & 35 & $2.216 \mathrm{e}-4$ & 4.572 & 52 & 19 \\
& G4L1 & $5.555 \mathrm{e}-7$ & 4.222 & 360 & 351 & $1.257 \mathrm{e}-5$ & 4.139 & 136 & 150 \\
& G5L1 & $3.269 \mathrm{e}-8$ & 4.086 & 100000 & 367964 & $7.668 \mathrm{e}-7$ & 4.035 & 687 & 2456 \\
\hline \multirow{5}{*}{ BICG } & G1L1 & $2.921 \mathrm{e}-3$ & & 20 & 2 & $2.519 \mathrm{e}-1$ & & 15 & 2 \\
& G2L1 & $2.261 \mathrm{e}-4$ & 3.691 & 36 & 7 & $5.273 \mathrm{e}-3$ & 5.578 & 24 & 4 \\
& G3L3 & $1.036 \mathrm{e}-5$ & 4.446 & 71 & 26 & $2.216 \mathrm{e}-4$ & 4.572 & 43 & 15 \\
& G4L1 & $5.555 \mathrm{e}-7$ & 4.222 & 135 & 152 & $1.257 \mathrm{e}-5$ & 4.139 & 81 & 84 \\
& G5L1 & $3.269 \mathrm{e}-8$ & 4.086 & 279 & 1118 & $7.668 \mathrm{e}-7$ & 4.035 & 153 & 582 \\
\hline
\end{tabular}

Tabela A.14: Erro e ordem de convergência dos métodos MDF de quarta ordem e ECDF de quarta ordem em malha uniforme com ordem do polinômio interpolador igual a 4

\begin{tabular}{c|c|cccc|cccc}
\hline $\begin{array}{c}\text { Solver } \\
(\text { tol } 1 e-16)\end{array}$ & Malha & \multicolumn{3}{c|}{ MDF } & \multicolumn{4}{c}{ ECDF } \\
& & $\|$ erro $\|_{2}$ & $\log _{2} r_{e} \approx q$ & Niter & Time & $\|$ erro $\|_{2}$ & $\log _{2} r_{e} \approx q$ & Niter & Time \\
& G1L1 & $3.124 \mathrm{e}-3$ & & 23 & 2 & $2.519 \mathrm{e}-1$ & & 14 & 1 \\
GMRES & G2L1 & $3.016 \mathrm{e}-4$ & 3.372 & 41 & 6 & $5.301 \mathrm{e}-3$ & 5.570 & 23 & 3 \\
& G3L3 & $1.262 \mathrm{e}-5$ & 4.578 & 108 & 36 & $2.233 \mathrm{e}-4$ & 4.569 & 52 & 17 \\
& G4L1 & $6.099 \mathrm{e}-7$ & 4.371 & 298 & 320 & $1.263 \mathrm{e}-5$ & 4.143 & 132 & 137 \\
& G5L1 & $3.387 \mathrm{e}-8$ & 4.170 & 100000 & 373040 & $7.686 \mathrm{e}-7$ & 4.039 & 415 & 1492 \\
\hline \multirow{5}{*}{ BICG } & G1L1 & $3.124 \mathrm{e}-3$ & & 20 & 2 & $2.519 \mathrm{e}-1$ & & 15 & 1 \\
& G2L1 & $3.016 \mathrm{e}-4$ & 3.372 & 36 & 7 & $5.301 \mathrm{e}-3$ & 5.570 & 24 & 4 \\
& G3L3 & $1.262 \mathrm{e}-5$ & 4.578 & 71 & 29 & $2.233 \mathrm{e}-4$ & 4.569 & 43 & 16 \\
& G4L1 & $6.099 \mathrm{e}-7$ & 4.371 & 146 & 189 & $1.263 \mathrm{e}-5$ & 4.143 & 81 & 92 \\
& G5L1 & $3.387 \mathrm{e}-8$ & 4.170 & 274 & 1151 & $7.686 \mathrm{e}-7$ & 4.039 & 153 & 623 \\
\hline
\end{tabular}

Tabela A.15: Erro e ordem de convergência dos métodos MDF de quarta ordem e $E C D F$ de quarta ordem em malha uniforme com ordem do polinômio interpolador igual a 5

\section{A.2.2 Tolerância igual a $1 e-13$ e ordem do polinômio intepolador variando em 3,4 e 5}




\begin{tabular}{c|l|cccc|cccc}
\hline $\begin{array}{c}\text { Solver } \\
\text { (tol } 1 e-13)\end{array}$ & Malha & \multicolumn{3}{|c|}{ MDF } & \multicolumn{3}{c}{ ECDF } \\
\hline \multirow{5}{*}{ GMRES } & G1L1 & $\begin{array}{l}\| \text { erro } \|_{2} \\
\log _{2} r_{e} \approx q\end{array}$ & Niter & Time & $\|$ erro $\|_{2}$ & $\log _{2} r_{e} \approx q$ & Niter & Time \\
\hline & G2L1 & $6.292 \mathrm{e}-4$ & 3.677 & 30 & 4 & $5.211 \mathrm{e}-3$ & 4.564 & 20 & 3 \\
& G3L3 & $3.411 \mathrm{e}-5$ & 4.204 & 86 & 27 & $2.201 \mathrm{e}-4$ & 4.564 & 42 & 12 \\
& G4L1 & $2.215 \mathrm{e}-6$ & 3.944 & 212 & 208 & $1.250 \mathrm{e}-5$ & 4.137 & 106 & 105 \\
& G5L1 & $1.426 \mathrm{e}-7$ & 3.956 & 543 & 1977 & $7.638 \mathrm{e}-7$ & 4.033 & 262 & 942 \\
\hline \multirow{5}{*}{ BICG } & G1L1 & $8.051-3$ & & 17 & 2 & $2.500 \mathrm{e}-1$ & & 13 & 2 \\
& G2L1 & $6.292 \mathrm{e}-4$ & 3.677 & 32 & 5 & $5.211 \mathrm{e}-3$ & 5.584 & 21 & 4 \\
& G3L3 & $3.411 \mathrm{e}-5$ & 4.204 & 61 & 22 & $2.201 \mathrm{e}-4$ & 4.564 & 37 & 12 \\
& G4L1 & $2.215 \mathrm{e}-6$ & 3.944 & 118 & 139 & $1.250 \mathrm{e}-5$ & 4.137 & 70 & 79 \\
& G5L1 & $1.426 \mathrm{e}-7$ & 3.956 & 230 & 925 & $7.638 \mathrm{e}-7$ & 4.033 & 133 & 525 \\
\hline
\end{tabular}

Tabela A.16: Erro e ordem de convergência dos métodos MDF de quarta ordem e ECDF de quarta ordem em malha uniforme com ordem do polinômio interpolador igual a 3

\begin{tabular}{|c|c|c|c|c|c|c|c|c|c|}
\hline \multirow{2}{*}{$\begin{array}{c}\text { Solver } \\
\text { (tol } 1 e-13)\end{array}$} & \multirow[t]{2}{*}{ Malha } & \multicolumn{4}{|c|}{ MDF } & \multicolumn{4}{|c|}{ ECDF } \\
\hline & & $\|$ erro $\|_{2}$ & $\log _{2} r_{e} \approx q$ & Niter & Time & $\|$ erro $\|_{2}$ & $\log _{2} r_{e} \approx q$ & Niter & Time \\
\hline \multirow{5}{*}{ GMRES } & G1L1 & $2.921 \mathrm{e}-3$ & & 17 & 2 & $2.519 \mathrm{e}-1$ & & 13 & 1 \\
\hline & G2L1 & $2.261 \mathrm{e}-4$ & 3.691 & 31 & 5 & $5.273 \mathrm{e}-3$ & 5.578 & 20 & 3 \\
\hline & G3L3 & $1.036 \mathrm{e}-5$ & 4.446 & 61 & 23 & $2.216 \mathrm{e}-4$ & 4.572 & 37 & 13 \\
\hline & G4L1 & $5.555 \mathrm{e}-7$ & 4.222 & 118 & 137 & $1.257 \mathrm{e}-5$ & 4.139 & 70 & 73 \\
\hline & G5L1 & $3.269 \mathrm{e}-8$ & 4.086 & 227 & 927 & $7.668 \mathrm{e}-7$ & 4.035 & 133 & 508 \\
\hline \multirow{5}{*}{ BICG } & G1L1 & $2.921 \mathrm{e}-3$ & & 20 & 2 & $2.519 \mathrm{e}-1$ & & 15 & 2 \\
\hline & G2L1 & $2.261 \mathrm{e}-4$ & 3.691 & 36 & 7 & $5.273 \mathrm{e}-3$ & 5.578 & 24 & 4 \\
\hline & G3L3 & $1.036 \mathrm{e}-5$ & 4.446 & 71 & 26 & $2.216 \mathrm{e}-4$ & 4.572 & 43 & 15 \\
\hline & G4L1 & $5.555 \mathrm{e}-7$ & 4.222 & 135 & 152 & $1.257 \mathrm{e}-5$ & 4.139 & 81 & 84 \\
\hline & G5L1 & $3.269 \mathrm{e}-8$ & 4.086 & 279 & 1118 & $7.668 \mathrm{e}-7$ & 4.035 & 153 & 582 \\
\hline
\end{tabular}

Tabela A.17: Erro e ordem de convergência dos métodos MDF de quarta ordem e ECDF de quarta ordem em malha uniforme com ordem do polinômio interpolador igual a 4 


\begin{tabular}{c|l|cccc|cccc}
\hline $\begin{array}{c}\text { Solver } \\
\text { (tol 1e-13) }\end{array}$ & Malha & \multicolumn{3}{c|}{ MDF } & \multicolumn{4}{c}{ ECDF } \\
\hline \multirow{5}{*}{ GMRES } & G1L1 & $3.124 \mathrm{e}-3$ & & & 16 & 2 & $2.519 \mathrm{e}-1$ & & \\
$\log _{2} r_{e} \approx q$ & Niter & Time & $\|$ erro $\|_{2} \approx q$ & Niter & Time \\
\hline & G2L1 & $3.016 \mathrm{e}-4$ & 3.372 & 29 & 6 & $5.301 \mathrm{e}-3$ & 5.570 & 20 & 3 \\
& G3L3 & $1.262 \mathrm{e}-5$ & 4.578 & 85 & 29 & $2.233 \mathrm{e}-4$ & 4.569 & 42 & 14 \\
& G4L1 & $6.099 \mathrm{e}-7$ & 4.371 & 203 & 226 & $1.263 \mathrm{e}-5$ & 4.143 & 106 & 110 \\
& G5L1 & $3.387 \mathrm{e}-8$ & 4.170 & 489 & 1838 & $7.686 \mathrm{e}-7$ & 4.039 & 271 & 976 \\
\hline \multirow{5}{*}{ BICG } & G1L1 & $3.124 \mathrm{e}-3$ & & 17 & 2 & $2.519 \mathrm{e}-1$ & & 13 & 1 \\
& G2L1 & $3.016 \mathrm{e}-4$ & 3.372 & 32 & 6 & $5.301 \mathrm{e}-3$ & 5.570 & 20 & 4 \\
& G3L3 & $1.262 \mathrm{e}-5$ & 4.578 & 61 & 26 & $2.233 \mathrm{e}-4$ & 4.569 & 37 & 14 \\
& G4L1 & $6.099 \mathrm{e}-7$ & 4.371 & 117 & 147 & $1.263 \mathrm{e}-5$ & 4.143 & 70 & 86 \\
& G5L1 & $3.387 \mathrm{e}-8$ & 4.170 & 227 & 968 & $7.686 \mathrm{e}-7$ & 4.039 & 133 & 536 \\
\hline
\end{tabular}

Tabela A.18: Erro e ordem de convergência dos métodos MDF de quarta ordem e ECDF de quarta ordem em malha uniforme com ordem do polinômio interpolador igual a 5

\section{A.2.3 Tolerância igual a $1 e-10$ e ordem do polinômio intepolador variando em 3,4 e 5}

\begin{tabular}{c|l|cccc|cccc}
\hline $\begin{array}{c}\text { Solver } \\
\text { tol 1e-10) }\end{array}$ & Malha & \multicolumn{3}{c|}{ MDF } & \multicolumn{3}{c}{ ECDF } \\
\hline \multirow{5}{*}{ GMRES } & G1L1 & $8.051 \mathrm{e}-3$ & & & 13 & 2 & $2.500 \mathrm{e}-1$ & & \\
$\log _{2} r_{e} \approx q$ & Niter & Time & $\|$ erro $\|_{2} r_{e} \approx q$ & Niter & Time \\
\hline & G2L1 & $6.292 \mathrm{e}-4$ & 3.677 & 25 & 4 & $5.211 \mathrm{e}-3$ & 4.564 & 17 & 3 \\
& G3L3 & $3.411 \mathrm{e}-5$ & 4.204 & 64 & 20 & $2.201 \mathrm{e}-4$ & 4.564 & 30 & 10 \\
& G4L1 & $2.215 \mathrm{e}-6$ & 3.944 & 151 & 157 & $1.250 \mathrm{e}-5$ & 4.137 & 82 & 76 \\
& G5L1 & $1.426 \mathrm{e}-7$ & 3.956 & 382 & 1387 & $7.638 \mathrm{e}-7$ & 4.033 & 185 & 679 \\
\hline \multirow{5}{*}{ BICG } & G1L1 & $8.051-3$ & & 15 & 2 & $2.500 \mathrm{e}-1$ & & 11 & 1 \\
& G2L1 & $6.292 \mathrm{e}-4$ & 3.677 & 26 & 4 & $5.211 \mathrm{e}-3$ & 5.584 & 17 & 3 \\
& G3L3 & $3.411 \mathrm{e}-5$ & 4.204 & 51 & 19 & $2.201 \mathrm{e}-4$ & 4.564 & 31 & 11 \\
& G4L1 & $2.215 \mathrm{e}-6$ & 3.944 & 101 & 115 & $1.250 \mathrm{e}-5$ & 4.137 & 58 & 68 \\
& G5L1 & $1.426 \mathrm{e}-7$ & 3.956 & 195 & 766 & $7.638 \mathrm{e}-7$ & 4.033 & 111 & 425 \\
\hline
\end{tabular}

Tabela A.19: Erro e ordem de convergência dos métodos MDF de quarta ordem e ECDF de quarta ordem em malha uniforme com ordem do polinômio interpolador igual a 3 


\begin{tabular}{|c|c|c|c|c|c|c|c|c|c|}
\hline \multirow{2}{*}{$\begin{array}{c}\text { Solver } \\
(\text { tol } 1 e-10)\end{array}$} & Malha & \multicolumn{4}{|c|}{ MDF } & \multicolumn{4}{|c|}{ ECDF } \\
\hline & & $\|$ erro $\|_{2}$ & $\log _{2} r_{e} \approx q$ & Niter & Time & $\|$ erro $\|_{2}$ & $\log _{2} r_{e} \approx q$ & Niter & Time \\
\hline \multirow{5}{*}{ GMRES } & G1L1 & $2.921 \mathrm{e}-3$ & & 13 & 2 & $2.519 \mathrm{e}-1$ & & 10 & 2 \\
\hline & G2L1 & $2.261 \mathrm{e}-4$ & 3.691 & 25 & 4 & $5.273 \mathrm{e}-3$ & 5.578 & 17 & 3 \\
\hline & G3L3 & $1.036 \mathrm{e}-5$ & 4.446 & 64 & 20 & $2.216 \mathrm{e}-4$ & 4.572 & 30 & 10 \\
\hline & G4L1 & $5.555 \mathrm{e}-7$ & 4.222 & 147 & 146 & $1.257 \mathrm{e}-5$ & 4.139 & 81 & 76 \\
\hline & G5L1 & $3.269 \mathrm{e}-8$ & 4.086 & 388 & 1401 & $7.668 \mathrm{e}-7$ & 4.035 & 197 & 699 \\
\hline \multirow{5}{*}{$\mathrm{BICG}$} & G1L1 & $2.921 \mathrm{e}-3$ & & 14 & 2 & $2.519 \mathrm{e}-1$ & & 11 & 1 \\
\hline & G2L1 & $2.261 \mathrm{e}-4$ & 3.691 & 26 & 5 & $5.273 \mathrm{e}-3$ & 5.578 & 17 & 3 \\
\hline & G3L3 & $1.036 \mathrm{e}-5$ & 4.446 & 51 & 19 & $2.216 \mathrm{e}-4$ & 4.572 & 31 & 11 \\
\hline & G4L1 & $5.555 \mathrm{e}-7$ & 4.222 & 101 & 116 & $1.257 \mathrm{e}-5$ & 4.139 & 58 & 62 \\
\hline & G5L1 & $3.269 \mathrm{e}-8$ & 4.086 & 192 & 769 & $7.668 \mathrm{e}-7$ & 4.035 & 111 & 441 \\
\hline
\end{tabular}

Tabela A.20: Erro e ordem de convergência dos métodos MDF de quarta ordem e ECDF de quarta ordem em malha uniforme com ordem do polinômio interpolador igual a 4

\begin{tabular}{c|l|cccc|cccc}
\hline $\begin{array}{c}\text { Solver } \\
(\text { tol } 1 e-10)\end{array}$ & Malha & \multicolumn{3}{|c|}{ MDF } & \multicolumn{4}{c}{ ECDF } \\
\hline \multirow{5}{*}{ GMRES } & G1L1 & $3.124 \mathrm{e}-3$ & & & 13 & 2 & $2.519 \mathrm{e}-1$ & & \\
$\log _{2} r_{e} \approx q$ & Niter & Time & $\|$ erro $\|_{2} r_{e} \approx q$ & Niter & Time \\
\hline & G2L1 & $3.016 \mathrm{e}-4$ & 3.372 & 25 & 4 & $5.301 \mathrm{e}-3$ & 5.570 & 17 & 3 \\
& G3L3 & $1.262 \mathrm{e}-5$ & 4.578 & 64 & 22 & $2.233 \mathrm{e}-4$ & 4.569 & 30 & 10 \\
& G4L1 & $6.099 \mathrm{e}-7$ & 4.371 & 148 & 165 & $1.263 \mathrm{e}-5$ & 4.143 & 81 & 79 \\
& G5L1 & $3.387 \mathrm{e}-8$ & 4.170 & 358 & 1332 & $7.686 \mathrm{e}-7$ & 4.039 & 197 & 721 \\
\hline \multirow{5}{*}{ BICG } & G1L1 & $3.124 \mathrm{e}-3$ & & 14 & 2 & $2.519 \mathrm{e}-1$ & & 11 & 2 \\
& G2L1 & $3.016 \mathrm{e}-4$ & 3.372 & 26 & 5 & $5.301 \mathrm{e}-3$ & 5.570 & 17 & 3 \\
& G3L3 & $1.262 \mathrm{e}-5$ & 4.578 & 53 & 23 & $2.233 \mathrm{e}-4$ & 4.569 & 31 & 12 \\
& G4L1 & $6.099 \mathrm{e}-7$ & 4.371 & 99 & 125 & $1.263 \mathrm{e}-5$ & 4.143 & 58 & 66 \\
& G5L1 & $3.387 \mathrm{e}-8$ & 4.170 & 190 & 819 & $7.686 \mathrm{e}-7$ & 4.039 & 111 & 451 \\
\hline
\end{tabular}

Tabela A.21: Erro e ordem de convergência dos métodos MDF de quarta ordem e ECDF de quarta ordem em malha uniforme com ordem do polinômio interpolador igual a 5

\section{A.2.4 Tolerância igual a $1 e-7$ e ordem do polinômio intepolador variando em 3,4 e 5}




\begin{tabular}{|c|c|c|c|c|c|c|c|c|c|}
\hline \multirow{2}{*}{$\begin{array}{c}\text { Solver } \\
\text { (tol } 1 e-7)\end{array}$} & \multirow[t]{2}{*}{ Malha } & \multicolumn{4}{|c|}{ MDF } & \multicolumn{4}{|c|}{ ECDF } \\
\hline & & $\|$ erro $\|_{2}$ & $\log _{2} r_{e} \approx q$ & Niter & Time & $\|$ erro $\|_{2}$ & $\log _{2} r_{e} \approx q$ & Niter & Time \\
\hline \multirow{5}{*}{ GMRES } & G1L1 & $8.051 \mathrm{e}-3$ & & 10 & 2 & $2.500 \mathrm{e}-1$ & & 8 & 1 \\
\hline & G2L1 & $6.292 \mathrm{e}-4$ & 3.677 & 20 & 3 & $5.211 \mathrm{e}-3$ & 4.564 & 13 & 3 \\
\hline & G3L3 & $3.411 \mathrm{e}-5$ & 4.204 & 42 & 14 & $2.201 \mathrm{e}-4$ & 4.564 & 24 & 8 \\
\hline & G4L1 & $2.215 \mathrm{e}-6$ & 3.944 & 90 & 91 & $1.250 \mathrm{e}-5$ & 4.137 & 52 & 55 \\
\hline & G5L1 & $2.211 \mathrm{e}-7$ & 3.956 & 237 & 851 & $7.638 \mathrm{e}-7$ & 4.033 & 128 & 452 \\
\hline \multirow{5}{*}{$\mathrm{BICG}$} & G1L1 & $8.051-3$ & & 12 & 1 & $2.500 \mathrm{e}-1$ & & 8 & 1 \\
\hline & G2L1 & $6.292 \mathrm{e}-4$ & 3.677 & 21 & 4 & $5.211 \mathrm{e}-3$ & 5.584 & 14 & 2 \\
\hline & G3L3 & $3.411 \mathrm{e}-5$ & 4.204 & 42 & 16 & $2.201 \mathrm{e}-4$ & 4.564 & 25 & 9 \\
\hline & G4L1 & $2.215 \mathrm{e}-6$ & 3.944 & 78 & 89 & $1.250 \mathrm{e}-5$ & 4.137 & 46 & 49 \\
\hline & G5L1 & $1.426 \mathrm{e}-7$ & 3.957 & 150 & 605 & $7.638 \mathrm{e}-7$ & 4.033 & 88 & 338 \\
\hline
\end{tabular}

Tabela A.22: Erro e ordem de convergência dos métodos MDF de quarta ordem e ECDF de quarta ordem em malha uniforme com ordem do polinômio interpolador igual a 3

\begin{tabular}{|c|c|c|c|c|c|c|c|c|c|}
\hline \multirow{2}{*}{$\begin{array}{c}\text { Solver } \\
(\text { tol } 1 e-7)\end{array}$} & \multirow[t]{2}{*}{ Malha } & \multicolumn{4}{|c|}{ MDF } & \multicolumn{4}{|c|}{ ECDF } \\
\hline & & $\|$ erro $\|_{2}$ & $\log _{2} r_{e} \approx q$ & Niter & Time & $\|$ erro $\|_{2}$ & $\log _{2} r_{e} \approx q$ & Niter & Time \\
\hline \multirow{5}{*}{ GMRES } & G1L1 & $2.921 \mathrm{e}-3$ & & 10 & 1 & $2.519 \mathrm{e}-1$ & & 8 & 1 \\
\hline & G2L1 & $2.261 \mathrm{e}-4$ & 3.691 & 20 & 4 & $5.273 \mathrm{e}-3$ & 5.578 & 13 & 2 \\
\hline & G3L3 & $1.036 \mathrm{e}-5$ & 4.446 & 42 & 13 & $2.216 \mathrm{e}-4$ & 4.572 & 24 & 8 \\
\hline & G4L1 & $5.555 \mathrm{e}-7$ & 4.222 & 90 & 91 & $1.257 \mathrm{e}-5$ & 4.139 & 52 & 50 \\
\hline & G5L1 & $1.975 \mathrm{e}-7$ & 1.498 & 233 & 858 & $7.668 \mathrm{e}-7$ & 4.035 & 128 & 456 \\
\hline \multirow{5}{*}{ BICG } & G1L1 & $2.921 \mathrm{e}-3$ & & 12 & 1 & $2.519 \mathrm{e}-1$ & & 8 & 2 \\
\hline & G2L1 & $2.261 \mathrm{e}-4$ & 3.691 & 21 & 4 & $5.273 \mathrm{e}-3$ & 5.578 & 14 & 3 \\
\hline & G3L3 & $1.036 \mathrm{e}-5$ & 4.446 & 41 & 15 & $2.216 \mathrm{e}-4$ & 4.572 & 25 & 9 \\
\hline & G4L1 & $5.555 \mathrm{e}-7$ & 4.222 & 77 & 87 & $1.257 \mathrm{e}-5$ & 4.139 & 46 & 53 \\
\hline & G5L1 & $3.269 \mathrm{e}-8$ & 4.086 & 150 & 603 & $7.668 \mathrm{e}-7$ & 4.035 & 88 & 343 \\
\hline
\end{tabular}

Tabela A.23: Erro e ordem de convergência dos métodos MDF de quarta ordem e ECDF de quarta ordem em malha uniforme com ordem do polinômio interpolador igual a 4 


\begin{tabular}{|c|c|c|c|c|c|c|c|c|c|}
\hline \multirow{2}{*}{$\begin{array}{c}\text { Solver } \\
(\text { tol } 1 e-7) \\
\end{array}$} & \multirow[t]{2}{*}{ Malha } & \multicolumn{4}{|c|}{ MDF } & \multicolumn{4}{|c|}{ ECDF } \\
\hline & & $\|$ erro $\|_{2}$ & $\log _{2} r_{e} \approx q$ & Niter & Time & $\|$ erro $\|_{2}$ & $\log _{2} r_{e} \approx q$ & Niter & Time \\
\hline \multirow{5}{*}{ GMRES } & G1L1 & $3.124 \mathrm{e}-3$ & & 10 & 1 & $2.519 \mathrm{e}-1$ & & 8 & 1 \\
\hline & G2L1 & $3.016 \mathrm{e}-4$ & 3.372 & 20 & 4 & $5.301 \mathrm{e}-3$ & 5.570 & 13 & 3 \\
\hline & G3L3 & $1.262 \mathrm{e}-5$ & 4.578 & 44 & 15 & $2.233 \mathrm{e}-4$ & 4.569 & 24 & 8 \\
\hline & G4L1 & $6.099 \mathrm{e}-7$ & 4.371 & 90 & 98 & $1.263 \mathrm{e}-5$ & 4.143 & 52 & 51 \\
\hline & G5L1 & $1.896 \mathrm{e}-7$ & 1.720 & 219 & 836 & $7.686 \mathrm{e}-7$ & 4.039 & 128 & 469 \\
\hline \multirow{5}{*}{ BICG } & G1L1 & $3.124 \mathrm{e}-3$ & & 12 & 2 & $2.519 \mathrm{e}-1$ & & 8 & 2 \\
\hline & G2L1 & $3.016 \mathrm{e}-4$ & 3.372 & 21 & 5 & $5.301 \mathrm{e}-3$ & 5.570 & 14 & 3 \\
\hline & G3L3 & $1.262 \mathrm{e}-5$ & 4.578 & 42 & 19 & $2.233 \mathrm{e}-4$ & 4.569 & 25 & 10 \\
\hline & G4L1 & $6.099 \mathrm{e}-7$ & 4.371 & 78 & 130 & $1.263 \mathrm{e}-5$ & 4.143 & 46 & 53 \\
\hline & G5L1 & $3.387 \mathrm{e}-8$ & 4.170 & 150 & 642 & $7.686 \mathrm{e}-7$ & 4.039 & 88 & 353 \\
\hline
\end{tabular}

Tabela A.24: Erro e ordem de convergência dos métodos MDF de quarta ordem e ECDF de quarta ordem em malha uniforme com ordem do polinômio interpolador igual a 5 


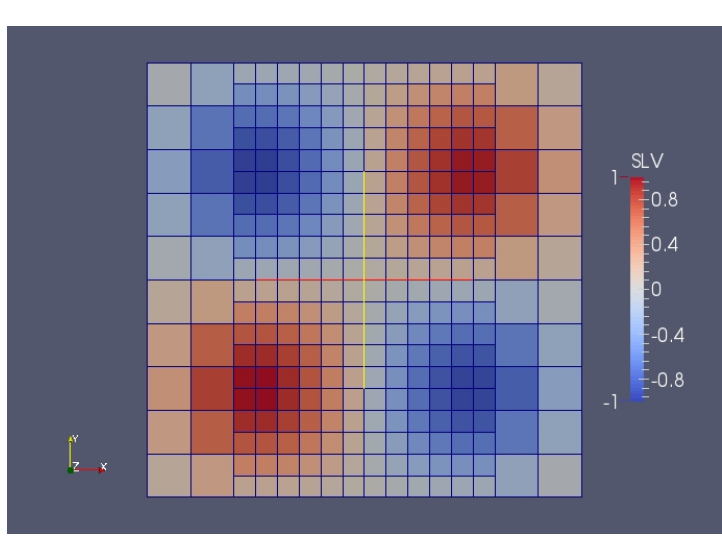

(a)

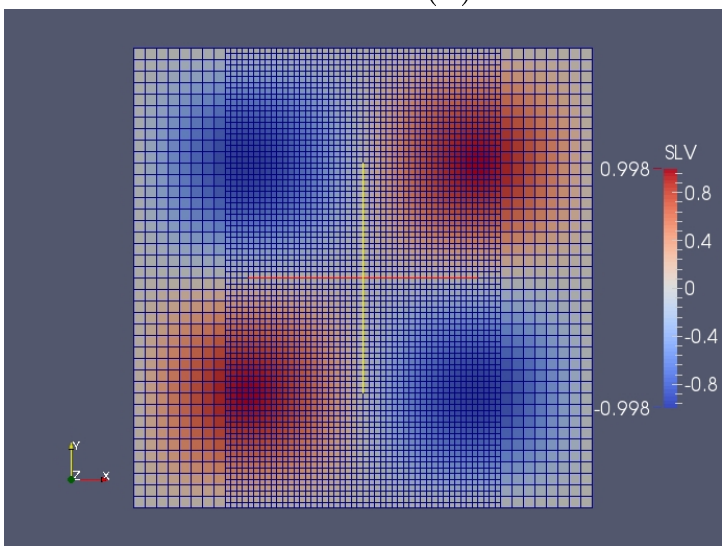

(c)

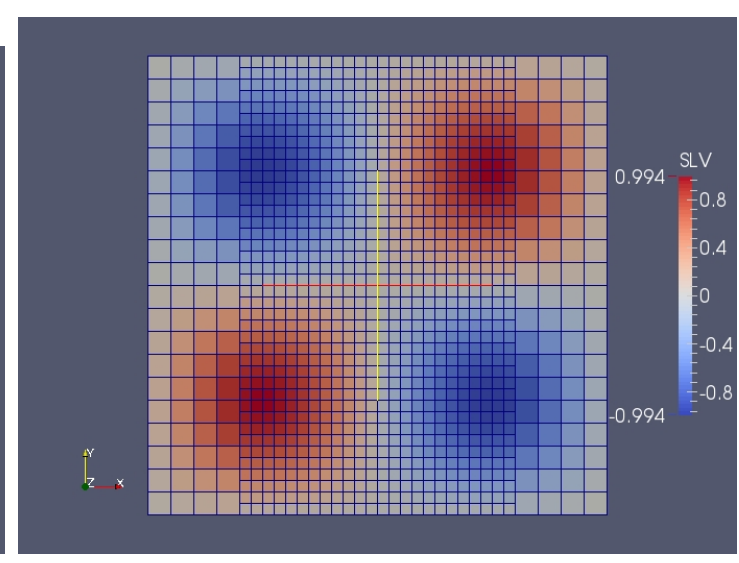

(b)

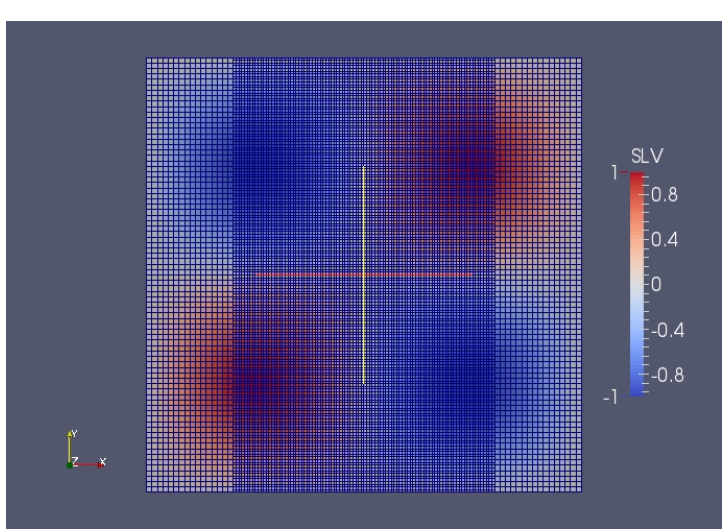

(d)

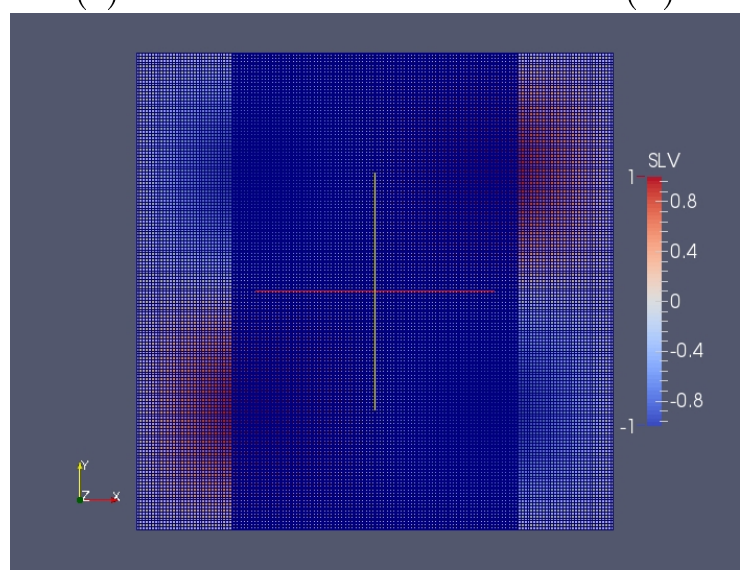

(e)

Figura A.3: (a) Malha G1L2, (b) Malha G2L2, (c) Malha G3L2 e (d) Malha G4L2 e (e) Malha G5L2 


\section{A.2.5 Tolerância igual a $1 e-16$ e ordem do polinômio intepolador variando em 3,4 e 5}

\begin{tabular}{c|l|lccc|cccc}
\hline $\begin{array}{c}\text { Solver } \\
(\text { tol } 1 e-16)\end{array}$ & Malha & \multicolumn{3}{c|}{ MDF } & \multicolumn{3}{c}{ ECDF } \\
& & $\|$ erro $\|_{2}$ & $\log _{2} r_{e} \approx q$ & Niter & Time & $\|$ erro $\|_{2}$ & $\log _{2} r_{e} \approx q$ & Niter & Time \\
\hline \multirow{5}{*}{ GMRES } & G1L2 & $1.098 \mathrm{e}-2$ & & 35 & 5 & $9.591 \mathrm{e}-3$ & & 21 & 3 \\
& G2L2 & $1.902 \mathrm{e}-3$ & 2.529 & 84 & 25 & $1.453 \mathrm{e}-3$ & 2.722 & 48 & 14 \\
& G3L3 & $2.401 \mathrm{e}-4$ & 2.986 & 237 & 199 & $1.987 \mathrm{e}-4$ & 2.870 & 120 & 99 \\
& G4L2 & $2.994 \mathrm{e}-5$ & 3.003 & 63059 & 182505 & $2.540 \mathrm{e}-5$ & 2.967 & 628 & 1849 \\
& G5L2 & $3.737 \mathrm{e}-6$ & 3.002 & 100000 & 1005526 & $3.179 \mathrm{e}-6$ & 2.998 & 100000 & 109952 \\
\hline \multirow{5}{*}{ BICG } & G1L2 & $1.098 \mathrm{e}-2$ & & 32 & 6 & $9.591 \mathrm{e}-3$ & & 21 & 4 \\
& G2L2 & $1.902 \mathrm{e}-3$ & 2.529 & 59 & 23 & $1.453 \mathrm{e}-3$ & 2.722 & 41 & 14 \\
& G3L3 & $2.401 \mathrm{e}-4$ & 2.986 & 133 & 138 & $1.987 \mathrm{e}-4$ & 2.870 & 80 & 73 \\
& G4L2 & $2.994 \mathrm{e}-5$ & 3.003 & 247 & 833 & $2.540 \mathrm{e}-5$ & 2.967 & 152 & 460 \\
& G5L2 & $3.737 \mathrm{e}-6$ & 3.002 & 522 & 5909 & $3.179 \mathrm{e}-6$ & 2.998 & 314 & 3452 \\
\hline
\end{tabular}

Tabela A.25: Erro e ordem de convergência dos métodos MDF de quarta ordem e ECDF de quarta ordem em malha composta com ordem do polinômio interpolador igual a 3

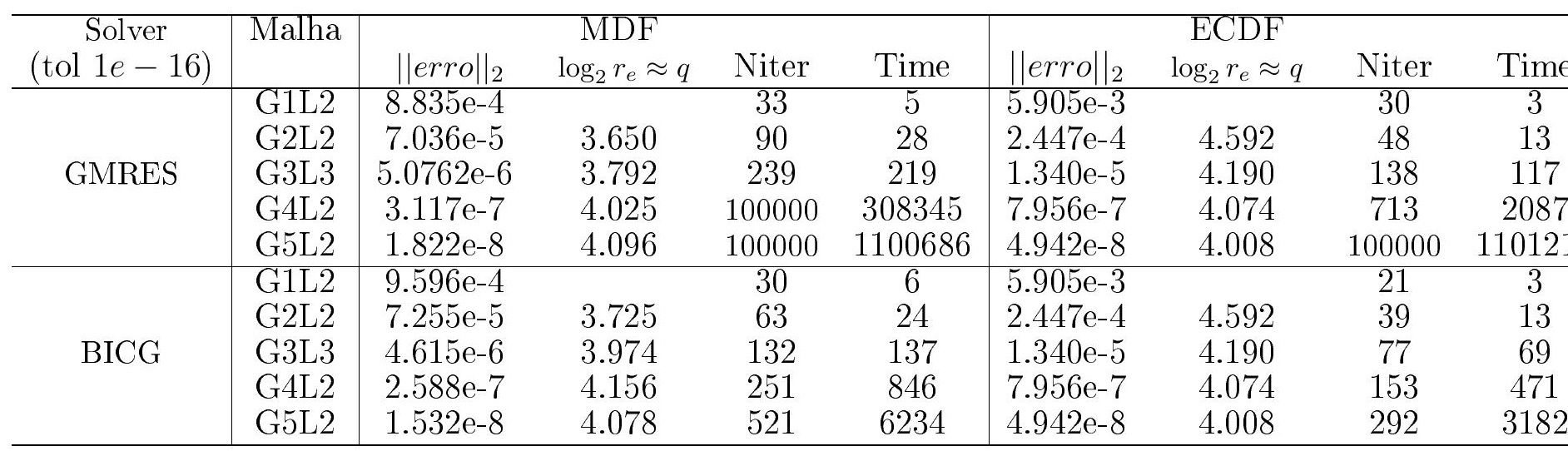

Tabela A.26: Erro e ordem de convergência dos métodos MDF de quarta ordem e ECDF de quarta ordem em malha composta com ordem do polinômio interpolador igual a 4 


\begin{tabular}{|c|c|c|c|c|c|c|c|c|c|}
\hline $\begin{array}{c}\text { Solver } \\
(\text { tol } 1 e-16)\end{array}$ & Malha & $\|$ erro $\|_{2}$ & $\begin{array}{c}\text { MDF } \\
\log _{2} r_{e} \approx q\end{array}$ & Niter & Time & $\|$ erro $\|_{2}$ & $\begin{array}{c}\text { ECDF } \\
\log _{2} r_{e} \approx q\end{array}$ & Niter & \\
\hline \multirow{5}{*}{ GMRES } & G1L2 & $1.224 \mathrm{e}-3$ & & 36 & 6 & $6.215 \mathrm{e}-3$ & & 26 & \\
\hline & G2L2 & $1.523 \mathrm{e}-4$ & 3.006 & 89 & 31 & $2.367 \mathrm{e}-4$ & 4.714 & 48 & \\
\hline & G3L3 & $1.011 \mathrm{e}-5$ & 3.913 & 209 & 223 & 4.168 & 4.062 & 133 & \\
\hline & G4L2 & $6.606 \mathrm{e}-7$ & 3.935 & 100000 & 348345 & $7.875 \mathrm{e}-7$ & 4.063 & 532 & \\
\hline & G5L2 & $3.916 \mathrm{e}-8$ & 4.076 & 100000 & 1137807 & $4.892 \mathrm{e}-8$ & 4.008 & 100000 & 111 \\
\hline \multirow{5}{*}{ BICG } & G1L2 & $1.275 \mathrm{e}-3$ & & 29 & 6 & $6.215 \mathrm{e}-3$ & & 21 & \\
\hline & G2L2 & $1.341 \mathrm{e}-4$ & 3.249 & 59 & 28 & $2.367 \mathrm{e}-4$ & 4.714 & 38 & \\
\hline & G3L3 & $8.061 \mathrm{e}-6$ & 4.056 & 126 & 159 & $1.316 \mathrm{e}-5$ & 4.168 & 73 & \\
\hline & G4L2 & $4.634 \mathrm{e}-7$ & 4.120 & 245 & 904 & $7.875 \mathrm{e}-7$ & 4.063 & 153 & \\
\hline & G5L2 & $2.769 \mathrm{e}-8$ & 4.064 & 499 & 5982 & $4.892 \mathrm{e}-8$ & 4.008 & 314 & \\
\hline
\end{tabular}

Tabela A.27: Erro e ordem de convergência dos métodos MDF de quarta ordem e ECDF de quarta ordem em malha composta com ordem do polinômio interpolador igual a 5

\section{A.2.6 Tolerância igual a $1 e-13$ e ordem do polinômio intepolador variando em 3,4 e 5}

\begin{tabular}{c|l|cccc|cccc}
\hline $\begin{array}{c}\text { Solver } \\
\text { (tol } 1 e-13)\end{array}$ & Malha & \multicolumn{3}{|c|}{ MDF } & \multicolumn{4}{c}{ ECDF } \\
\hline \multirow{5}{*}{ GMRES } & G1L2 & $1.098 \mathrm{e}-2$ & & & & \\
$\log _{2} r_{e} \approx q$ & Niter & Time & $\|$ erro $\|_{2}$ & $\log _{2} r_{e} \approx q$ & Niter & Time \\
\hline & G2L2 & $1.902 \mathrm{e}-3$ & 2.529 & 79 & 4 & $9.591 \mathrm{e}-3$ & & 18 & 2 \\
& G3L3 & $2.401 \mathrm{e}-4$ & 2.986 & 176 & 160 & $1.453 \mathrm{e}-3$ & 2.722 & 36 & 11 \\
& G4L2 & $2.994 \mathrm{e}-5$ & 3.003 & 537 & 1675 & $2.540 \mathrm{e}-4$ & 2.870 & 101 & 82 \\
& G5L2 & $3.737 \mathrm{e}-6$ & 3.002 & 1153 & 12925 & $3.179 \mathrm{e}-6$ & 2.967 & 254 & 730 \\
& G1L2 & $1.098 \mathrm{e}-2$ & & 27 & 5 & $9.591 \mathrm{e}-3$ & & 603 & 6528 \\
\hline \multirow{5}{*}{ BICG } & G2L2 & $1.902 \mathrm{e}-3$ & 2.529 & 55 & 22 & $1.453 \mathrm{e}-3$ & 2.722 & 35 & 12 \\
& G3L3 & $2.401 \mathrm{e}-4$ & 2.986 & 111 & 125 & $1.987 \mathrm{e}-4$ & 2.870 & 65 & 64 \\
& G4L2 & $2.994 \mathrm{e}-5$ & 3.003 & 214 & 791 & $2.540 \mathrm{e}-5$ & 2.967 & 130 & 428 \\
& G5L2 & $3.737 \mathrm{e}-6$ & 3.002 & 442 & 5450 & $3.179 \mathrm{e}-6$ & 2.998 & 260 & 3119 \\
\hline
\end{tabular}

Tabela A.28: Erro e ordem de convergência dos métodos MDF de quarta ordem e ECDF de quarta ordem em malha composta com ordem do polinômio interpolador igual a 3 


\begin{tabular}{c|l|cccc|cccc}
\hline $\begin{array}{c}\text { Solver } \\
(\text { tol } 1 e-13)\end{array}$ & Malha & \multicolumn{3}{c|}{ MDF } & \multicolumn{3}{c}{ ECDF } \\
& & $\|$ erro $\|_{2}$ & $\log _{2} r_{e} \approx q$ & Niter & Time & $\|$ erro $\|_{2}$ & $\log _{2} r_{e} \approx q$ & Niter & Time \\
\hline \multirow{5}{*}{ GMRES } & G1L2 & $8.835 \mathrm{e}-4$ & & 26 & 5 & $5.905 \mathrm{e}-3$ & & 18 & 3 \\
& G2L2 & $7.036 \mathrm{e}-5$ & 3.650 & 78 & 24 & $2.447 \mathrm{e}-4$ & 4.592 & 36 & 10 \\
& G3L3 & $5.0762 \mathrm{e}-6$ & 3.792 & 178 & 162 & $1.340 \mathrm{e}-5$ & 4.190 & 99 & 82 \\
& G4L2 & $3.117 \mathrm{e}-7$ & 4.025 & 540 & 1665 & $7.956 \mathrm{e}-7$ & 4.074 & 237 & 700 \\
& G5L2 & $1.822 \mathrm{e}-8$ & 4.096 & 1196 & 13381 & $4.942 \mathrm{e}-8$ & 4.008 & 611 & 6693 \\
\hline \multirow{5}{*}{ BICG } & G1L2 & $9.596 \mathrm{e}-4$ & & 27 & 4 & $5.905 \mathrm{e}-3$ & & 18 & 4 \\
& G2L2 & $7.255 \mathrm{e}-5$ & 3.725 & 54 & 22 & $2.447 \mathrm{e}-4$ & 4.592 & 36 & 13 \\
& G3L3 & $4.615 \mathrm{e}-6$ & 3.974 & 113 & 128 & $1.340 \mathrm{e}-5$ & 4.190 & 66 & 65 \\
& G4L2 & $2.588 \mathrm{e}-7$ & 4.156 & 229 & 820 & $7.956 \mathrm{e}-7$ & 4.074 & 129 & 427 \\
& G5L2 & $1.532 \mathrm{e}-8$ & 4.078 & 442 & 5497 & $4.942 \mathrm{e}-8$ & 4.008 & 255 & 3070 \\
\hline
\end{tabular}

Tabela A.29: Erro e ordem de convergência dos métodos MDF de quarta ordem e ECDF de quarta ordem em malha composta com ordem do polinômio interpolador igual a 4

\begin{tabular}{c|l|cccc|cccc}
\hline $\begin{array}{c}\text { Solver } \\
(\text { tol } 1 e-13)\end{array}$ & Malha & \multicolumn{3}{|c|}{ MDF } & \multicolumn{4}{c}{ ECDF } \\
& & $\|$ erro $\|_{2}$ & $\log _{2} r_{e} \approx q$ & Niter & Time & $\|$ erro $\|_{2}$ & $\log _{2} r_{e} \approx q$ & Niter & Time \\
\hline \multirow{5}{*}{ GMRES } & G1L2 & $1.224 \mathrm{e}-3$ & & 25 & 4 & $6.215 \mathrm{e}-3$ & & 18 & 4 \\
& G2L2 & $1.523 \mathrm{e}-4$ & 3.006 & 78 & 27 & $2.367 \mathrm{e}-4$ & 4.714 & 36 & 12 \\
& G3L3 & $1.011 \mathrm{e}-5$ & 3.913 & 168 & 180 & 4.168 & 4.062 & 99 & 88 \\
& G4L2 & $6.606 \mathrm{e}-7$ & 3.935 & 509 & 1719 & $7.875 \mathrm{e}-7$ & 4.063 & 255 & 783 \\
& G5L2 & $3.916 \mathrm{e}-8$ & 4.076 & 987 & 11184 & $4.892 \mathrm{e}-8$ & 4.008 & 605 & 6995 \\
\hline \multirow{5}{*}{ BICG } & G1L2 & $1.275 \mathrm{e}-3$ & & 27 & 6 & $6.215 \mathrm{e}-3$ & & 19 & 4 \\
& G2L2 & $1.341 \mathrm{e}-4$ & 3.249 & 54 & 25 & $2.367 \mathrm{e}-4$ & 4.714 & 35 & 14 \\
& G3L3 & $8.061 \mathrm{e}-6$ & 4.056 & 113 & 158 & $1.316 \mathrm{e}-5$ & 4.168 & 67 & 75 \\
& G4L2 & $4.634 \mathrm{e}-7$ & 4.120 & 229 & 911 & $7.875 \mathrm{e}-7$ & 4.063 & 133 & 485 \\
& G5L2 & $2.769 \mathrm{e}-8$ & 4.064 & 456 & 6010 & $4.892 \mathrm{e}-8$ & 4.008 & 270 & 3417 \\
\hline
\end{tabular}

Tabela A.30: Erro e ordem de convergência dos métodos MDF de quarta ordem e ECDF de quarta ordem em malha composta com ordem do polinômio interpolador igual a 5

\section{A.2.7 Tolerância igual a $1 e-10$ e ordem do polinômio intepolador variando em 3,4 e 5}




\begin{tabular}{c|c|cccc|cccc}
\hline $\begin{array}{c}\text { Solver } \\
\text { tol 1e-10) }\end{array}$ & Malha & \multicolumn{3}{|c|}{ MDF } & \multicolumn{3}{c}{ ECDF } \\
\hline \multirow{5}{*}{ GMRES } & G1L2 & $1.098 \mathrm{e}-2$ & & & 22 & 3 & $9.591 \mathrm{e}-3$ & & \\
$\log _{2} r_{e} \approx q$ & Niter & Time & $\|$ erro $\|_{2}$ & $r_{e} \approx q$ & Niter & Time \\
\hline & G2L2 & $1.902 \mathrm{e}-3$ & 2.529 & 57 & 18 & $1.453 \mathrm{e}-3$ & 2.722 & 28 & 8 \\
& G3L3 & $2.401 \mathrm{e}-4$ & 2.986 & 127 & 116 & $1.987 \mathrm{e}-4$ & 2.870 & 77 & 71 \\
& G4L2 & $2.994 \mathrm{e}-5$ & 3.003 & 415 & 1304 & $2.540 \mathrm{e}-5$ & 2.967 & 166 & 481 \\
& G5L2 & $3.737 \mathrm{e}-6$ & 3.002 & 826 & 9080 & $3.179 \mathrm{e}-6$ & 2.998 & 420 & 4627 \\
\hline \multirow{5}{*}{ BICG } & G1L2 & $1.098 \mathrm{e}-2$ & & 24 & 5 & $9.591 \mathrm{e}-3$ & & 16 & 3 \\
& G2L2 & $1.902 \mathrm{e}-3$ & 2.529 & 48 & 19 & $1.453 \mathrm{e}-3$ & 2.722 & 30 & 11 \\
& G3L3 & $2.401 \mathrm{e}-4$ & 2.986 & 97 & 111 & $1.987 \mathrm{e}-4$ & 2.870 & 55 & 57 \\
& G4L2 & $2.994 \mathrm{e}-5$ & 3.003 & 187 & 679 & $2.540 \mathrm{e}-5$ & 2.967 & 110 & 364 \\
& G5L2 & $3.737 \mathrm{e}-6$ & 3.002 & 355 & 4514 & $3.179 \mathrm{e}-6$ & 2.998 & 216 & 2627 \\
\hline
\end{tabular}

Tabela A.31: Erro e ordem de convergência dos métodos MDF de quarta ordem e ECDF de quarta ordem em malha composta com ordem do polinômio interpolador igual a 3

\begin{tabular}{c|l|cccc|cccc}
\hline $\begin{array}{c}\text { Solver } \\
\text { (tol 1e-10) }\end{array}$ & Malha & \multicolumn{3}{c|}{ MDF } & \multicolumn{3}{c}{ ECDF } \\
& G1L2 & $8.835 \mathrm{e}-4$ & & & 22 & 4 & $5.905 \mathrm{e}-3$ & & \\
$\log _{2} r_{e} \approx q$ & Niter & Time & $\|$ erro $\|_{2} \approx q$ & Niter & Time \\
\hline \multirow{5}{*}{ GMRES } & G2L2 & $7.036 \mathrm{e}-5$ & 3.650 & 57 & 18 & $2.447 \mathrm{e}-4$ & 4.592 & 28 & 9 \\
& G3L3 & $5.0762 \mathrm{e}-6$ & 3.792 & 129 & 117 & $1.340 \mathrm{e}-5$ & 4.190 & 77 & 63 \\
& G4L2 & $3.117 \mathrm{e}-7$ & 4.025 & 412 & 1299 & $7.956 \mathrm{e}-7$ & 4.074 & 157 & 455 \\
& G5L2 & $1.822 \mathrm{e}-8$ & 4.096 & 777 & 8653 & $4.942 \mathrm{e}-8$ & 4.008 & 476 & 5179 \\
\hline \multirow{5}{*}{ BICG } & G1L2 & $9.596 \mathrm{e}-4$ & & 24 & 4 & $5.905 \mathrm{e}-3$ & & 16 & 3 \\
& G2L2 & $7.255 \mathrm{e}-5$ & 3.725 & 48 & 19 & $2.447 \mathrm{e}-4$ & 4.592 & 31 & 11 \\
& G3L3 & $4.615 \mathrm{e}-6$ & 3.974 & 94 & 106 & $1.340 \mathrm{e}-5$ & 4.190 & 56 & 55 \\
& G4L2 & $2.588 \mathrm{e}-7$ & 4.156 & 185 & 684 & $7.956 \mathrm{e}-7$ & 4.074 & 110 & 364 \\
& G5L2 & $1.532 \mathrm{e}-8$ & 4.078 & 355 & 4401 & $4.942 \mathrm{e}-8$ & 4.008 & 212 & 2597 \\
\hline
\end{tabular}

Tabela A.32: Erro e ordem de convergência dos métodos MDF de quarta ordem e ECDF de quarta ordem em malha composta com ordem do polinômio interpolador igual a 4 


\begin{tabular}{|c|c|c|c|c|c|c|c|c|c|}
\hline \multirow{2}{*}{$\begin{array}{c}\text { Solver } \\
(\text { tol } 1 e-10)\end{array}$} & Malha & \multicolumn{4}{|c|}{ MDF } & \multicolumn{4}{|c|}{ ECDF } \\
\hline & & $\|$ erro $\|_{2}$ & $\log _{2} r_{e} \approx q$ & Niter & Time & $\|$ erro $\|_{2}$ & $\log _{2} r_{e} \approx q$ & Niter & Time \\
\hline \multirow{5}{*}{ GMRES } & G1L2 & $1.275 \mathrm{e}-3$ & & 22 & 4 & $6.215 \mathrm{e}-3$ & & 15 & 3 \\
\hline & G2L2 & $1.341 \mathrm{e}-4$ & 3.249 & 57 & 21 & $2.367 \mathrm{e}-4$ & 4.714 & 28 & 10 \\
\hline & G3L3 & $1.011 \mathrm{e}-5$ & 4.056 & 120 & 131 & $1.316 \mathrm{e}-5$ & 4.168 & 77 & 68 \\
\hline & G4L2 & $6.606 \mathrm{e}-7$ & 4.120 & 389 & 1292 & $7.875 \mathrm{e}-7$ & 4.063 & 179 & 572 \\
\hline & G5L2 & $3.916 \mathrm{e}-8$ & 4.064 & 696 & 7553 & $4.892 \mathrm{e}-8$ & 4.008 & 436 & 4828 \\
\hline \multirow{5}{*}{$\mathrm{BICG}$} & G1L2 & $1.275 \mathrm{e}-3$ & & 24 & 5 & $6.215 \mathrm{e}-3$ & & 16 & 4 \\
\hline & G2L2 & $1.341 \mathrm{e}-4$ & 3.249 & 48 & 23 & $2.367 \mathrm{e}-4$ & 4.714 & 30 & 12 \\
\hline & G3L3 & $1.011 \mathrm{e}-5$ & 4.056 & 97 & 136 & $1.316 \mathrm{e}-5$ & 4.168 & 55 & 62 \\
\hline & G4L2 & $6.606 \mathrm{e}-7$ & 4.120 & 177 & 726 & $7.875 \mathrm{e}-7$ & 4.063 & 112 & 406 \\
\hline & G5L2 & $3.916 \mathrm{e}-8$ & 4.064 & 355 & 4677 & $4.892 \mathrm{e}-8$ & 4.008 & 216 & 2830 \\
\hline
\end{tabular}

Tabela A.33: Erro e ordem de convergência dos métodos MDF de quarta ordem e ECDF de quarta ordem em malha composta com ordem do polinômio interpolador igual a 5

\section{A.2.8 Tolerância igual a $1 e-7$ e ordem do polinômio intepolador variando em 3,4 e 5}

\begin{tabular}{|c|c|c|c|c|c|c|c|c|c|}
\hline \multirow{2}{*}{$\begin{array}{c}\text { Solver } \\
(\text { tol } 1 e-7)\end{array}$} & \multirow[t]{2}{*}{ Malha } & \multicolumn{4}{|c|}{ MDF } & \multicolumn{4}{|c|}{ ECDF } \\
\hline & & $\|$ erro $\|_{2}$ & $\log _{2} r_{e} \approx q$ & Niter & Time & $\|$ erro $\|_{2}$ & $\log _{2} r_{e} \approx q$ & Niter & Time \\
\hline \multirow{5}{*}{ GMRES } & G1L2 & $1.098 \mathrm{e}-2$ & & 17 & 3 & $9.591 \mathrm{e}-3$ & & 12 & 2 \\
\hline & G2L2 & $1.902 \mathrm{e}-3$ & 2.529 & 43 & 14 & $1.453 \mathrm{e}-3$ & 2.722 & 23 & 7 \\
\hline & G3L3 & $2.401 \mathrm{e}-4$ & 2.986 & 82 & 79 & $1.987 \mathrm{e}-4$ & 2.870 & 55 & 50 \\
\hline & G4L2 & $2.994 \mathrm{e}-5$ & 3.003 & 289 & 925 & $2.540 \mathrm{e}-5$ & 2.967 & 106 & 309 \\
\hline & G5L2 & $3.737 \mathrm{e}-6$ & 3.002 & 462 & 5319 & $3.179 \mathrm{e}-6$ & 2.998 & 275 & 3154 \\
\hline \multirow{5}{*}{ BICG } & G1L2 & $1.098 \mathrm{e}-2$ & & 20 & 4 & $9.591 \mathrm{e}-3$ & & 13 & 2 \\
\hline & G2L2 & $1.902 \mathrm{e}-3$ & 2.529 & 37 & 16 & $1.453 \mathrm{e}-3$ & 2.722 & 25 & 9 \\
\hline & G3L3 & $2.401 \mathrm{e}-4$ & 2.986 & 75 & 87 & $1.987 \mathrm{e}-4$ & 2.870 & 47 & 48 \\
\hline & G4L2 & $2.994 \mathrm{e}-5$ & 3.003 & 150 & 565 & $2.540 \mathrm{e}-5$ & 2.967 & 91 & 302 \\
\hline & G5L2 & $3.737 \mathrm{e}-6$ & 3.002 & 289 & 3580 & $3.179 \mathrm{e}-6$ & 2.998 & 176 & 2159 \\
\hline
\end{tabular}

Tabela A.34: Erro e ordem de convergência dos métodos MDF de quarta ordem e ECDF de quarta ordem em malha composta com ordem do polinômio interpolador igual a 3 


\begin{tabular}{c|c|cccc|cccc}
\hline $\begin{array}{c}\text { Solver } \\
\text { tol 1e-7) }\end{array}$ & Malha & \multicolumn{3}{c|}{ MDF } & \multicolumn{3}{c}{ ECDF } \\
& G1L2 & $8.835 \mathrm{e}-4$ & & & 17 & 3 & $5.905 \mathrm{e}-3$ & & \\
$\log _{2} r_{e} \approx q$ & Niter & Time & $\|$ erro $\|_{2} \approx q$ & Niter & Time \\
\hline \multirow{5}{*}{ GMRES } & G2L2 & $7.036 \mathrm{e}-5$ & 3.650 & 43 & 14 & $2.447 \mathrm{e}-4$ & 4.592 & 22 & 7 \\
& G3L3 & $5.0762 \mathrm{e}-6$ & 3.792 & 81 & 75 & $1.340 \mathrm{e}-5$ & 4.190 & 55 & 46 \\
& G4L2 & $3.117 \mathrm{e}-7$ & 4.025 & 274 & 875 & $7.956 \mathrm{e}-7$ & 4.074 & 106 & 314 \\
& G5L2 & $1.822 \mathrm{e}-8$ & 4.096 & 452 & 5113 & $4.942 \mathrm{e}-8$ & 4.008 & 295 & 3268 \\
\hline \multirow{5}{*}{ BICG } & G1L2 & $9.596 \mathrm{e}-4$ & & 20 & 4 & $5.905 \mathrm{e}-3$ & & 13 & 3 \\
& G2L2 & $7.255 \mathrm{e}-5$ & 3.725 & 37 & 16 & $2.447 \mathrm{e}-4$ & 4.592 & 24 & 9 \\
& G3L3 & $4.615 \mathrm{e}-6$ & 3.974 & 76 & 92 & $1.340 \mathrm{e}-5$ & 4.190 & 47 & 48 \\
& G4L2 & $2.588 \mathrm{e}-7$ & 4.156 & 147 & 537 & $7.956 \mathrm{e}-7$ & 4.074 & 87 & 293 \\
& G5L2 & $1.532 \mathrm{e}-8$ & 4.078 & 293 & 3824 & $4.942 \mathrm{e}-8$ & 4.008 & 179 & 2232 \\
\hline
\end{tabular}

Tabela A.35: Erro e ordem de convergência dos métodos MDF de quarta ordem e ECDF de quarta ordem em malha composta com ordem do polinômio interpolador igual a 4

\begin{tabular}{c|c|cccc|cccc}
\hline $\begin{array}{c}\text { Solver } \\
\text { tol 1e-7) }\end{array}$ & Malha & \multicolumn{3}{c|}{ MDF } & \multicolumn{3}{c}{ ECDF } \\
& & $\|$ erro $\|_{2}$ & $\log _{2} r_{e} \approx q$ & Niter & Time & $\|$ erro $\|_{2}$ & $\log _{2} r_{e} \approx q$ & Niter & Time \\
\hline \multirow{5}{*}{ GMRES } & G1L2 & $1.224 \mathrm{e}-3$ & & 18 & 4 & $6.215 \mathrm{e}-3$ & & 13 & 3 \\
& G2L2 & $1.523 \mathrm{e}-4$ & 3.006 & 37 & 18 & $2.367 \mathrm{e}-4$ & 4.714 & 25 & 11 \\
& G3L3 & $1.011 \mathrm{e}-5$ & 3.913 & 75 & 103 & 4.168 & 4.062 & 46 & 52 \\
& G4L2 & $6.606 \mathrm{e}-7$ & 3.935 & 151 & 595 & $7.875 \mathrm{e}-7$ & 4.063 & 91 & 323 \\
& G5L2 & $3.916 \mathrm{e}-8$ & 4.076 & 278 & 3662 & $4.892 \mathrm{e}-8$ & 4.008 & 179 & 2363 \\
\hline \multirow{5}{*}{ BICG } & G1L2 & $1.275 \mathrm{e}-3$ & & 17 & 3 & $6.215 \mathrm{e}-3$ & & 12 & 3 \\
& G2L2 & $1.341 \mathrm{e}-4$ & 3.249 & 43 & 16 & $2.367 \mathrm{e}-4$ & 4.714 & 23 & 8 \\
& G3L3 & $8.061 \mathrm{e}-6$ & 4.056 & 81 & 91 & $1.316 \mathrm{e}-5$ & 4.168 & 54 & 51 \\
& G4L2 & $4.634 \mathrm{e}-7$ & 4.120 & 270 & 1011 & $7.875 \mathrm{e}-7$ & 4.063 & 107 & 329 \\
& G5L2 & $2.769 \mathrm{e}-8$ & 4.064 & 418 & 5043 & $4.892 \mathrm{e}-8$ & 4.008 & 313 & 3669 \\
\hline
\end{tabular}

Tabela A.36: Erro e ordem de convergência dos métodos MDF de quarta ordem e ECDF de quarta ordem em malha composta com ordem do polinômio interpolador igual a 5 


\section{Referências Bibliográficas}

[1] Y. Adam. Highly Accurate Compact Implicit Methods and Boundary Conditions. Journal of Computational Physics, 24:10-22, 1977. 3

[2] C. M. R. Alverez. Simulação computacional adaptativa de escoamentos bisáficos viscoelásticos. PhD thesis, Tese de Doutorado, Instituto de Matemática e Estatística, Universidade de São Paulo, Brasil, 2013. 1, 5, 34

[3] U. Ananthakrishnaiah, R. Manohar, and J. W. Stephenson. High-Order Methods for Elliptic Equations With Variable Coefficients. Numerical Methods for Partial Diffecntial Equations, 3:219-227, 1987. 4

[4] J. B. Bell, P. Colella, and H. M. Glaz. A Second Order Projection Method for the Incompressible Navier-Stokes Equations. Journal of Computational Physics, 85:257283, 1989. 17

[5] P. C. Calegari. Simulações de escoamentos bifásicos tridimensionais empregando métodos adaptativos e modelos de campo de fase. $\mathrm{PhD}$ thesis, Tese de Doutorado, Instituto de Matemática e Estatística, Universidade de São Paulo, Brasil, 2013. 1

[6] A. Chorin and J. E. Marsden. A Mathematical Introduction to Fluid Mechanics. Springer-Verlag, 1990. 17

[7] A. J. Chorin. Numerical solution of the navier-stokes equations. 104:745-762, 1968. 5, 17

[8] L. Collatz. The numerical treatment of differential equations. Springer-Verlag, 1966. 3

[9] A. de Oliveira Fortuna. Técnicas Computacionais para Dinâmicas dos Fluidos. Conceitos Básicos e Aplicações. EDUSP, 2000. 1, 2, 15, 18

[10] F. S. de Sousa, N. Mangiavacchi, L. G. Nonato, A. C. Filho, M. F. Tomé, V. G. Ferreira, J. A. Cuminato, and S. McKee. A Front-Tracking/Front-Capturing Method for the Simulation of 3D Multi-Fluid Flows with Free Surfaces. Journal of Computational Physics. 198:469-499, 2004. 17

[11] W. E. and J.-G. Liu. Vorticity boundary condition and related issues for finite difference schemes. 124:368-382, 1996. 16

[12] E. A. Fadlun, R. Verzicco, P. Orlandi, and J. Mohd-Yusof. Combined Immersed Boundary Finite-Difference Methods for Three-Dimensional Complex Flow Simulations. Journal of Computational Physics, 161:35-60, 2000. 17

[13] L. Fauci and C. S. Peskin. A Computational Model of Aquatic Animal Locomotion. Journal of Computational Physics, 77:85-108, 1988. 17 
[14] L. Ge and J. Zhang. Accuracy, robustness and efficiency comparison in iterative computation of convection diffusion equation with boundary layers. 16:379?394, 2000. 4

[15] M. M. Gupta. High Accuracy Solutions of Incompressible Navier-Stokes Equations. Journal of computational physics, 93:343-359, 1991. 3, 4, 5

[16] M. M. Gupta, R. P. Manohar, and J. W. Stephenson. A Single Cell High Order Scheme For The Convection-Diffusion Equation With Vaiable Coefficients. International Journal For Numerical Methods In Fluids, 4:641-651, 1984. 4

[17] F. H. Harlow and J. E. Welch. Numerical calculation of time-dependent viscous of incompressible flow of fluid with free surface. 8:2182-2189, 1965.16

[18] R. S. Hirsch. Higher order accurate difference solution of fluid mechanics problems by a compact differencing technique. pages 90-109, 1975. 3

[19] J. V. Kan. A Second-Order Accurate Pressure-Correction Scheme for Viscous Incompressible Flow. SIAM Journal on Scientific and Statistical Computation, 7:870-891, 1986. 17

[20] S. Karaa. High-order difference schemes for 2D elliptic and parabolic problems with mixed derivatives. Numerical Methods for Partial Differential Equations, 23:366?378, 2007. 5

[21] G. Karniadakis, M. Israeli, and S. A. Orszag. High-order splitting methods for the incompressible navier-stokes equations. 97:414-443, 1991. 16

[22] J. Kim and P. Moin. Application of a Fractional-Step Method to Incompressible NavierStokes Equations. Journal of Computational Physics, 59:308-323, 1985. 17

[23] C. F. A. Lages. Métodos numéricos para escoamentos multifásicos em malhas hierárquicas. Master's thesis, Instituto de Ciências Matemáticas e de Computação, Universidade de São Paulo, Brasil, May 2016. 5, 7

[24] B. Lan, Y. Ge, Y. Wang, and Y. Zhan. High order compact difference scheme and multigrid method for $2 \mathrm{~d}$ elliptic problems with variable coefficients and interior/boundary layers on nonuniform grids. 2015. 18, 19

[25] H. P. Langtangen, K.-A. Mardal, and R. Winther. Numerical methods for incompressible viscous flow. 25:1125-1146, 2002. 16

[26] S. K. Lele. Compact finite difference schemes with spectral-like resolution. 1991. 3

[27] S. K. Lele. Compact finite difference schemes with spectral-like resolution. 103:16-42, 1992. 18

[28] R. J. Leveque. Finite Volume Methods for Hyperbolic Problems. Cambridge: Cambridge University Press, 2002. 18

[29] L. Li and H.-W. Shen. Image-Based Streamline Generation and Rendering. Visualization and computer graphics, 13:630-640, 2007. 1

[30] M. Li and T. Tang. A compact fourth-order finite difference scheme for the steady incompressible Navier-Stokes equations. International Journal For Numerical Methods In Fluids, 20:1137-1151, 1995. 3, 4, 5, 15 
[31] P. F. A. Mancera and R. Hunt. Fourth order method for solving the Navier?Stokes equations in a constricting channel. Int. J. Numer. Methods Fluids, 25:1119?1135, 1997. 4

[32] P. F. A. Mancera and R. Hunt. Some experiments with high order compact methods using a computer algebra software?Part I. Applied Mathematics and Computation, 174:775?794, 2006. 3, 4

[33] L. Ming-Chih. Simulation of the flow Past an Array of Circular Cylinders as a Test of the Immersed Boundary Method. PhD thesis, Ph.D. thesis, New York University, 1998. 17

[34] A. Nachbin. Aspectos de modelagem matemática em dinãmica dos fluidos. Master's thesis, IMPA - Instituto Nacional de Matem’atica Pura e Aplicada, 2001. 15

[35] J. C. Patterson. General Derivative Approximations for Finite Difference Schemes. International Journal for Numerical Methods in Engineering, 19:1235-1241, 1983. 3

[36] C. S. Peskin. Flow patterns around heart valves: A numerical method. 10:252-271, 1972. 17

[37] D. M. M. Queen and C. S. Peskin. A Three-Dimensional Computational Method for Blood Flow in the Heart. I. Immersed Elastic Fibers in a Viscous Incompressible Fluid. 81:372-405, 1989. 17

[38] D. M. M. Queen and C. S. Peskin. Heart simulation by an immersed boundary method with formal second-order accuracy and reduced numerical viscosity. 2000. 17

[39] S. A. E. Remigio. Simulação Numérica Bidimensional da Interação Fluido-Estrutura através do Método Físico Virtual. PhD thesis, Tese de Doutorado, Instituto de Matemática e Estatística, Universidade de São Paulo, Brasil, Setembro 2005. 16

[40] S Balay and J Brown and K Buschelman and V Eijkhout and W Gropp and D Kaushik and M Knepley and L C mcinnes and B Smith and H Zhang. PETSc User Manual 3.4. [S.l.], 2013. 26, 27

[41] H. L. Soriano. Método de Elementos Finitos em Análise de Estrutura. EDUSP, 2003. 18

[42] W. F. Spotz. High-Order Compact Finite Difference Schemes for Computational Mechanics. PhD thesis, The University of Texas at Austin, Dezembro 1995. 2, 3, 19, 21, 24

[43] A. I. Tolstykh. High accuracy non-centered compact difference schemes for fluid dynamics applications. 1994. 3

[44] M. M. Villar. Análise numérica detalhada de escoamentos multifásicos bidimensionais. PhD thesis, Tese de Doutorado, Universidade Federal de Uberlândia, Uberlândia, Brasil, 2007. 1,5

[45] D. M. Young and J. H. Dauwalder. Discrete Representations of Partial Differential Operators. Errors in Digital Computation, pages 181-207, 1965. 4 